\title{
Ultracold Neutral Plasmas
}

\author{
T. C. Killian ${ }^{\text {a }}$, T. Pattard ${ }^{\mathrm{b}}$, T. Pohl ${ }^{\mathrm{c}}$ and J. M. Rost ${ }^{\mathrm{d}}$ \\ ${ }^{a}$ Rice University, Department of Physics and Astronomy and Rice Quantum \\ Institute, Houston, Texas, USA \\ b APS Editorial Office, 1 Research Road, Ridge, NY 11961 \\ ' ITAMP, Harvard-Smithsonian Center for Astrophysics, 60 Garden Street, \\ Cambridge, MA 02138, USA \\ d Max Planck Institute for the Physics of Complex Systems, Dresden, Germany
}

\begin{abstract}
Ultracold neutral plasmas, formed by photoionizing laser-cooled atoms near the ionization threshold, have electron temperatures in the 1-1000 kelvin range and ion temperatures from tens of millikelvin to a few kelvin. They represent a new frontier in the study of neutral plasmas, which traditionally deals with much hotter systems, but they also blur the boundaries of plasma, atomic, condensed matter, and low temperature physics. Modelling these plasmas challenges computational techniques and theories of non-equilibrium systems, so the field has attracted great interest from the theoretical and computational physics communities. By varying laser intensities and wavelengths it is possible to accurately set the initial plasma density and energy, and charged-particle-detection and optical diagnostics allow precise measurements for comparison with theoretical predictions.

Recent experiments using optical probes demonstrated that ions in the plasma equilibrate in a strongly coupled fluid phase. Strongly coupled plasmas, in which the electrical interaction energy between charged particles exceeds the average kinetic energy, reverse the traditional energy hierarchy underlying basic plasma concepts such as Debye screening and hydrodynamics. Equilibration in this regime is of particular interest because it involves the establishment of spatial correlations between particles, and it connects to the physics of the interiors of gas-giant planets and inertial confinement fusion devices.
\end{abstract}

\section{Contents}

1 Introduction 3

2 Experimental Methods 6

2.1 Creation of ultracold neutral plasmas 6

Preprint submitted to Elsevier 24 August 2018 
2.2 Detection of ultracold neutral plasmas

3 Theoretical Description 16

3.1 Macroscopic Approaches 17

3.2 Microscopic Approaches 27

$4 \quad$ Physical Processes in Ultracold Neutral Plasmas 33

4.1 Initial Electron Equilibration 34

4.2 Initial Ion Equilibration 40

4.3 Collective Electronic Plasma Modes 46

4.4 Plasma Expansion 49

4.5 Electron Heating Mechanisms 56

4.6 Coulomb Coupling Parameters 60

$5 \quad$ Achieving Strong Coupling 64

5.1 Ionic coupling 65

5.2 Electronic coupling

6 Conclusions and Future Directions 71

A Important Quantities 73

B Extracting Plasma Parameters from the Absorption Spectrum 73

C The Quasineutral Approximation 75

\begin{tabular}{ll} 
References & 76 \\
\hline
\end{tabular} 


\section{Introduction}

In a conventional neutral plasma, ions and electrons are created from atoms and molecules by ionizing collisions between particles. Because a typical ionization potential is on the order of an electronvolt, most neutral plasmas have temperatures of thousands of kelvin or more. (See Fig. 1.) Plasma physics in this regime encompasses a wealth of fascinating fundamental phenomena such as collective modes, instabilities, and transport mechanisms, and it can contribute to important applications such as lighting technologies, plasma processing, and the pursuit of fusion energy.

Ultracold neutral plasmas [2] stretch the boundaries of traditional neutral plasma physics. They are formed by photoionizing laser-cooled atoms near the ionization threshold and have electron temperatures ranging from 1-1000 K and ion temperatures of around $1 \mathrm{~K}$. While providing access to a new regime, they have also proven to be clean and simple systems that form an excellent testing ground for basic plasma theory, and they elucidate physics found across a much wider spectrum of plasma energy and density.

The clearest distinguishing feature of ultracold neutral plasmas is that particles can be in or near the strongly coupled regime [3]. In strongly coupled plasmas the Coulomb interaction energy between charged particles exceeds the average kinetic energy, which is quantified by the Coulomb coupling parameter

$$
\Gamma=e^{2} /\left[4 \pi \varepsilon_{0} a k_{B} T\right],
$$

where $a=[3 /(4 \pi \rho)]^{1 / 3}$ is the Wigner-Seitz radius characterizing the separation between particles in a plasma of density $\rho$ and temperature $T$. $\Gamma>1$ reverses the traditional energy hierarchy that underlies our normal understanding of plasmas based on concepts such as Debye screening and hydrodynamics. Strongly coupled plasmas exist in dense astrophysical systems [4, matter irradiated with intense laser fields [5,6,7,8], colloidal or dusty plasmas of highly charged macroscopic particles [9, 10], and non-neutral trapped ion plasmas that are laser cooled until they freeze into Wigner crystals [1]. Strong coupling is manifested through the presence of spatial correlations between particles, which can be important for the equilibration and collective dynamics of the system. Optical probes [12,13] demonstrated that ions in an ultracold neutral plasma can be strongly coupled. Electrons seem to remain just outside of this regime because the rate of recombination of electrons and ions to form neutral particles diverges rapidly as the electron temperature drops.

There is great similarity between the dynamics of ultracold neutral plasmas and equilibration of plasmas created by fast-pulse laser irradiation of solid [14, 15] and thin film targets [16,17, 18, 19,20 21,22,23, 24], rare gas clusters [25], 


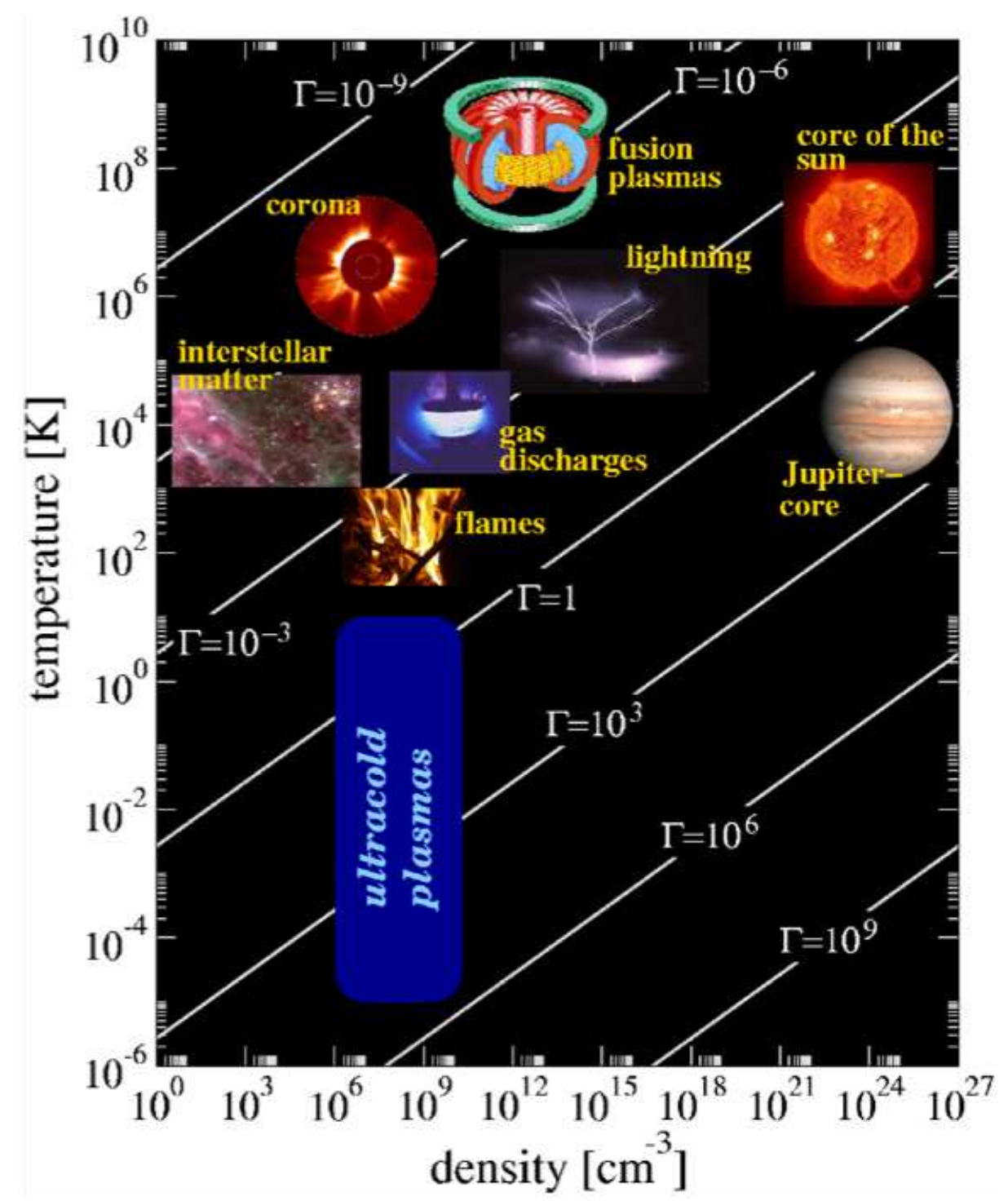

Fig. 1. Overview of neutral plasmas in the density-temperature parameter plane. Lines of constant Coulomb coupling parameter $\Gamma$ (see Eq. (1)) are indicated. After [1].

and gas jets [26 27]. However, the fast-pulse systems have densities close to or higher than the condensed phase. This complicates observation of plasma dynamics, which takes place on the scale of the inverse plasma frequency, $\omega_{\mathrm{p}}^{-1}=\left[m \varepsilon_{0} /\left(e^{2} \rho\right)\right]^{1 / 2}$, and is of the order of attoseconds for electron and femtoseconds for ions. Ultracold plasmas, on the other hand, reach the strong coupling regime despite the fact that they are extremely dilute (Fig. 1). The low density implies that ultracold plasmas evolve on a time scale that is more 
easily accessible experimentally. Studies of ion equilibration have revealed oscillations of the ion kinetic energy [28,29,30,31] that are a fundamental characteristic of equilibration of strongly coupled systems. Expansion of a plasma into the surrounding vacuum may be directly observed with excellent spatial and temporal resolution in ultracold systems $32,33,34,13$. This phenomenon is important in fast-pulse experiments, especially in the context of the generation of high energy particles [14,15,16,17,18,19,20,21,22, 23, 24, 25, 26, 27], inertialconfinement fusion experiments [35], and x-ray lasers [36].

High energy-density and strongly coupled plasmas are often difficult to describe theoretically because of the importance of many-body interactions, and these problems are often best addressed with computational techniques. Many body interactions are also important in ultracold neutral plasmas, and computational physicists have made significant contributions to the understanding of these system by applying techniques usually used for high energy-density systems [37,38]. Ultracold plasmas have motivated the development and application of specially adapted numerical techniques to describe the presence of spatial correlations between the ions [39] and the evolution of electrons and ions on very different timescales [37. Using these techniques it has even been possible to lead the experimental efforts by predicting the possibility of Wigner crystallization in an ultracold expanding neutral plasma [34] and the conditions that lead to it. Again, the excellent experimental control and diagnostics available imply that these computational results can be tested with great precision in ultracold systems.

Immediately after the creation of an ultracold neutral plasma through photoionization, the system is far from equilibrium, and most research in the field has focused on the equilibration process. Theoretical and computational work $33,37,38,40,41,42,43$, has identified processes that heat the plasma. For plasmas in or near the strongly coupled regime, where the potential energy is comparable to or exceeds the thermal energy, excess potential energy arising from a random distribution of ions [40] or electrons [41] is converted to thermal energy. This is called disorder-induced heating. Another important heating mechanism is three-body recombination (TBR), in which an ion and two electrons collide to form an energetic electron and an excited neutral atom. The TBR rate varies with temperature as $T^{-9 / 2}$ [44], and at low temperatures it can become the fastest process in the plasma. TBR is a crucial process for the formation of cold antihydrogen through positron-antiproton recombination [45,46]. Conditions in these anti-matter plasmas are similar to ultracold neutral plasmas except a large magnetic field is usually present to contain the particles. Surprisingly, recombination in ultracold neutral plasmas also has similarities to stellar dynamics in globular clusters [47].

The study of collective modes has proven to be a rich area of research, originally motivated by the possibility of observing modification of the mode 


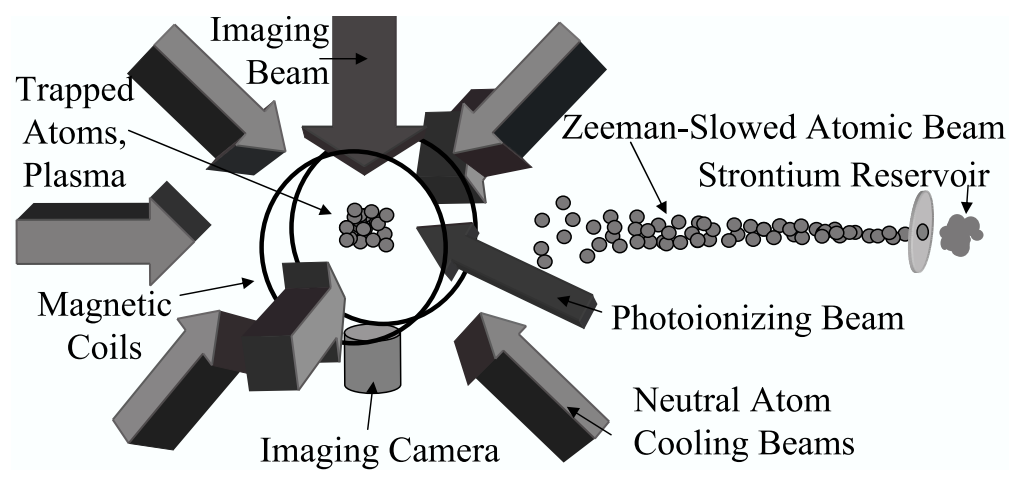

Fig. 2. Schematic setup of a typical ultracold plasma experiment, exemplified with strontium atoms. Neutral atoms are laser cooled and trapped in a magneto-optical trap operating on the ${ }^{1} S_{0}-{ }^{1} P_{1}$ transition at $461 \mathrm{~nm}$, as described in [51]. In a second step, ${ }^{1} P_{1}$ atoms are ionized by photons from a laser at $\sim 412 \mathrm{~nm}$ (see Fig. 3A). Finally, the ionic plasma component is imaged using the ${ }^{2} S_{1 / 2}-{ }^{2} P_{1 / 2}$ transition in $\mathrm{Sr}^{+}$at $422 \mathrm{~nm}$ (see Fig. 33B). Reused with permission from [12]. Copyright 2004, American Physical Society.

structure due to strong coupling [48]. Electron plasma oscillations [32,49] and Tonks-Dattner modes [50] have served as probes of plasma dynamics and represent interesting fundamental problems in their own right.

The review is structured such that Sections 2 and 3 provide the experimental and theoretical basis that allows one to understand and manipulate ultracold neutral plasmas. Section 4 of the review describes the physical processes which take place in an ultracold neutral plasma from the time it is created until it expands into the surrounding vacuum. In Section 5, we discuss proposals and prospects for achieving very strong coupling of the plasma - in some sense the "holy grail" of ultracold plasma physics. We conclude with Section 6 by discussing some possible future directions of this relatively young research field.

\section{Experimental Methods}

\subsection{Creation of ultracold neutral plasmas}

The production of an ultracold neutral plasma starts with laser-cooled and trapped neutral atoms. There are many good resources on this topic, such as the book "Laser Cooling and Trapping," by Metcalf and van der Straten [52]. In a configuration of laser beams and magnetic fields known as a magnetooptical trap (Fig. 2), as many as $10^{9}$ atoms can be cooled to millikelvin or even microkelvin temperatures, and confined at densities approaching $10^{11} \mathrm{~cm}^{-3}$. 


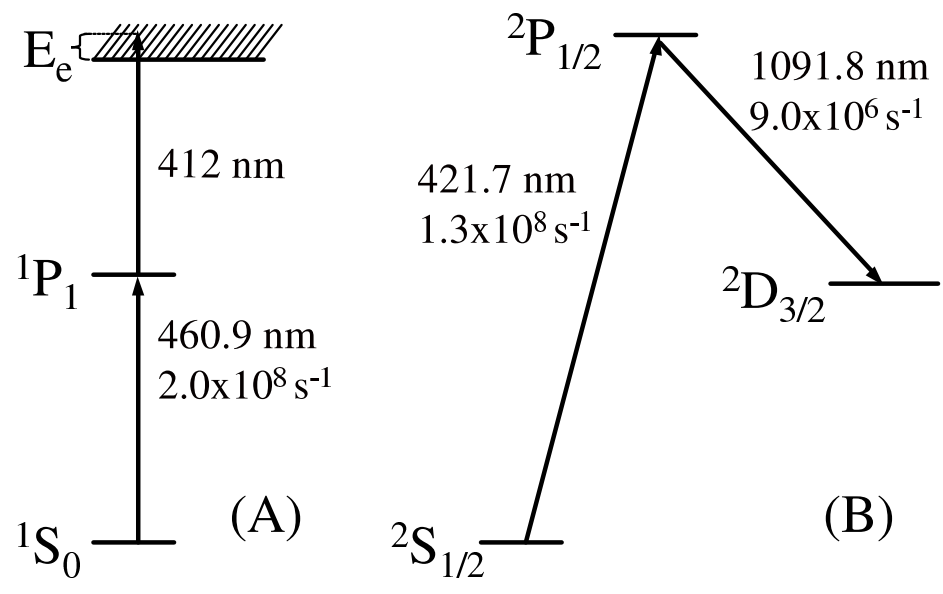

Fig. 3. Strontium atomic and ionic energy levels with decay rates relevant for ultracold plasma experiments. (A) Creation of an ultracold plasma starts with laser cooling and trapping neutral atoms using the ${ }^{1} S_{0}-{ }^{1} P_{1}$ transition at $461 \mathrm{~nm} .{ }^{1} P_{1}$ atoms are then photoionized with photons from a laser at $\sim 412 \mathrm{~nm}$. (B) Ions are optically imaged using the ${ }^{2} S_{1 / 2}-{ }^{2} P_{1 / 2}$ transition in $\mathrm{Sr}^{+}$at $422 \mathrm{~nm} .{ }^{2} P_{1 / 2}$ ions decay to the ${ }^{2} D_{3 / 2}$ state $7 \%$ of the time, after which they cease to interact with the imaging beam. This does not affect most experiments because ions typically scatter fewer than one photon while the imaging beam is on. The level scheme is similar for calcium [13].

The typical spatial distribution of the cloud is of spherical Gaussian shape,

$$
\rho(r)=\rho_{0} \exp \left(-r^{2} / 2 \sigma^{2}\right)
$$

with a width of $\sigma=200-1500 \mu \mathrm{m}$. The first ultracold plasma experiments were performed at the National Institute of Standards and Technology in Gaithersburg (NIST) with metastable xenon [53], but any atom that can be laser cooled and photoionized may be used, such as strontium [51,54, calcium [13], rubidium [55,56], and cesium [56]. A plasma has also been produced by photoionizing a Bose-Einstein condensate [57].

A pulsed dye laser excites the laser-cooled atoms above the ionization threshold (Fig. 3A ). Because of their light mass, the electrons have an initial kinetic energy $\left(E_{\mathrm{e}}\right)$ approximately equal to the difference between the photon energy and the ionization potential. $E_{\mathrm{e}} / k_{B}$ can be as low as the bandwidth of the ionizing laser, which is $\sim 100 \mathrm{mK}$ with standard pulsed dye lasers, but most studies so far have dealt with $E_{\mathrm{e}} / k_{B}$ between 1 and $1000 \mathrm{~K}$. The initial kinetic energy for the ions is in the millikelvin range. The number of atoms ionized $\left(N_{i}\right)$, and thus the density of the plasma $(\rho)$, is controlled by varying the energy of the photoionizing laser pulse.

We will discuss the dynamics of the plasma in greater detail in Section 4 , 


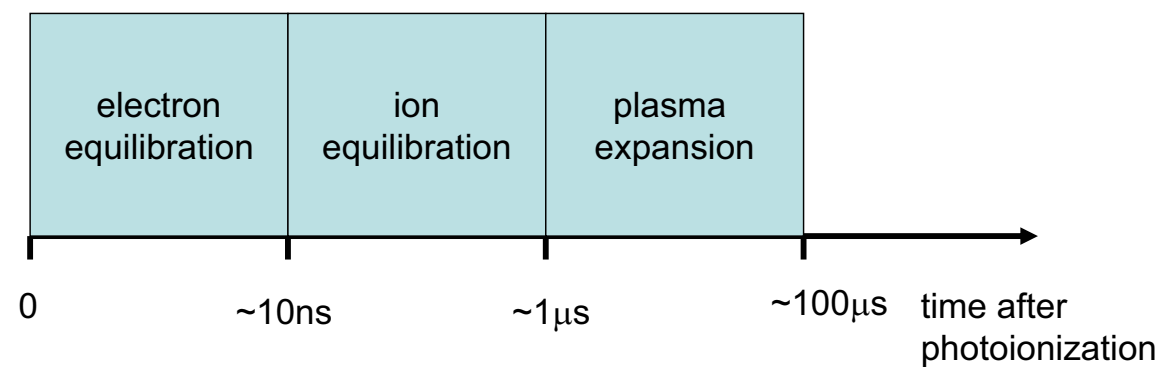

Fig. 4. Stages and timescales in the dynamics of an ultracold neutral plasma. Electron equilibration is a fast process in ultracold neutral plasmas, and it occurs in a time equal to the inverse electron plasma frequency, $\omega_{\mathrm{p}, \mathrm{e}}^{-1}=\sqrt{m_{\mathrm{e}} \varepsilon_{0} /\left(\rho_{\mathrm{e}} e^{2}\right)}$. Analogously, the timescale for ion equilibration in the second stage is the inverse ion plasma frequency, $\omega_{\mathrm{p}, \mathrm{i}}^{-1}=\sqrt{m_{\mathrm{i}} \varepsilon_{0} /\left(\rho_{\mathrm{i}} e^{2}\right)}$. The timescale for plasma expansion is set by the hydrodynamic time, $\tau_{\exp }=\sqrt{m_{\mathrm{i}} \sigma^{2} / k_{B} T_{\mathrm{e}}}$.

but Fig. 4 illustrates how the evolution can be divided into three phases. The fastest process is the equilibration of the electrons because they are relatively energetic and light. The more sluggish ions then equilibrate with themselves on a slightly longer timescale. And finally, the plasma - which is unconfined in typical experiments - expands into the surrounding vacuum.

\subsubsection{Photoionization}

The photoionization process is crucial in these experiments, and the book "Rydberg Atoms" [58] by T. Gallagher provides an excellent introduction to this subject. Most ultracold plasma experiments have used two-photon ionization, in which one photon from the continuous-wave (CW) cooling laser excites atoms to the upper level of the cooling transition, and a pulsed laser then excites them to the continuum. (See Fig. 3.)

In equilibrium the fraction of atoms in the upper state of the cooling transition can approach $50 \%$, and is given by [52]

$$
f=\frac{s_{0} / 2}{1+s_{0}+(2 \delta / \gamma)^{2}},
$$

where $\delta$ is the detuning from resonance of the $\mathrm{CW}$ laser, $\gamma$ is the full linewidth at half maximum for the transition, and $s_{0}=I / I_{\text {sat }}$ is the saturation parameter for the excitation beam of intensity $I . I_{\text {sat }}$ is typically a few $\mathrm{mW} / \mathrm{cm}^{2}$, and it is the intensity required to drive the transition at a rate equal to $\gamma$. The excited-state fraction during normal MOT operation is typically about $10 \%$, 
but an extra excitation beam may also be used to attain $f \approx 0.5$. Highly saturating the transition with a pulsed-dye-amplified beam yields even better results [13].

Photoionization from the intermediate state is best described in terms of a cross section for absorption of ionizing photons, $\sigma_{\mathrm{PI}}$. Cross sections near the ionization threshold are of the order of the atomic unit of area, $\sigma_{\mathrm{PI}} \approx a_{0}^{2} \approx$ $10^{-18} \mathrm{~cm}^{2}$. The probability of ionization is given by $1-\exp \left(-F \sigma_{\mathrm{PI}}\right)$, where the number of photons per unit area in the pulse is $F=\int_{\text {pulse }} d t I / h \nu_{\text {laser }}$ for instantaneous laser intensity $I$. A blue $(450 \mathrm{~nm}), 1 \mathrm{~mJ}, 5 \mathrm{~ns}$ pulse has about $2 \times 10^{15}$ photons, and an intensity of $20 \mathrm{MW} / \mathrm{cm}^{2}$ for a pulse area of $1 \mathrm{~mm}^{2}$. This gives a $2 \times 10^{17} \mathrm{~cm}^{-2}$ integrated photon flux and an ionization probability of $20 \%$ for atoms in the intermediate level. With more power it is possible to ionize nearly $100 \%$ of the atoms in the intermediate state.

Cross sections are normally constant over the near-threshold-ionization region of interest for ultracold plasmas, unless there is some physics feature that modifies them, such as an autoionizing resonance, which is the case in strontium [59]. In alkali metals except lithium, cross sections for single-photon excitation from the $S$ ground state to near the ionization threshold show Cooper minima [58. This is one reason why most ultracold plasma experiments use two-photon excitation.

The ionization process adds momentum and energy to the electrons and ions. The particles can be treated as at rest initially, and recoil during excitation to the intermediate state is negligible. Momentum and energy conservation for the pulsed laser photons of frequency $\nu$ gives

$$
\begin{gathered}
\Delta E \equiv h \nu-E_{I P}=\frac{p_{\mathrm{i}}^{2}}{2 m_{\mathrm{i}}}+\frac{p_{\mathrm{e}}^{2}}{2 m_{\mathrm{e}}}, \\
\hbar \mathbf{k}=\mathbf{p}_{\mathrm{i}}+\mathbf{p}_{\mathrm{e}},
\end{gathered}
$$

where the ionization potential is $E_{I P}$. The photon momentum $k=|\mathbf{k}|$ is small. For instance, at $10 \mathrm{~K}, \hbar k^{2} \approx\left\langle p_{\mathrm{e}}^{2}\right\rangle / 100$, which implies that $p_{\mathrm{i}}^{2} \approx p_{\mathrm{e}}^{2}$. The ion kinetic energy is thus much smaller than the electron kinetic energy, $E_{\mathrm{e}}$. Hence, the electrons take away essentially all the excess photon energy, $\Delta E$, as kinetic energy:

$$
E_{e} \equiv \frac{p_{\mathrm{e}}^{2}}{2 m_{\mathrm{e}}} \approx \Delta E
$$

The ion kinetic energy is then

$$
\frac{p_{\mathrm{i}}^{2}}{2 m_{\mathrm{i}}} \approx \frac{p_{\mathrm{e}}^{2}}{2 m_{\mathrm{i}}}=\Delta E \frac{m_{\mathrm{e}}}{m_{\mathrm{i}}},
$$

which is only on the order of millikelvin even for $\Delta E=k_{B} 1000 \mathrm{~K}$. Within a few nanoseconds after photoionization [60], the electrons collisionally thermalize 
with themselves at $k_{B} T_{\mathrm{e}} \approx \frac{2}{3} E_{\mathrm{e}}$. We will describe below how processes in the plasma can subsequently heat the electrons and ions to substantially higher temperatures.

\subsubsection{Spontaneous ionization of a dense Rydberg gas}

An ultracold neutral plasma also forms from spontaneous ionization in a dense cloud of ultracold Rydberg atoms. In fact, the first experiments focused on this phenomenon [61, and the hope was to observe a gas-phase analog of the Mott insulator-to-conductor transition familiar from condensed matter physics [62]. The idea was first explored by Haroche and co-workers [63] when they saw spontaneous ionization in a beam of Rydberg atoms.

In experiments performed so far, collisions, as opposed to a phase transition, drive the spontaneous ionization process in beams or laser-cooled clouds, and after the formation of the plasma, the dynamics is very similar to the evolution of a system created by direct photoionization [33]. We will touch upon this work briefly in Sec. 4.1.2. However, it will not be the main focus of this review, and for a deeper discussion we refer to papers exploring the spontaneous evolution of Rydberg atoms into a plasma [64] and dynamics of Rydberg atoms embedded in an ultracold plasma [55]. Related questions regarding the stability of a Rydberg gas are important for fundamental interest and also because this is a proposed architecture for quantum computing [65].

\subsection{Detection of ultracold neutral plasmas}

\subsubsection{Charged particle detection}

The most common diagnostics for studying ultracold plasmas is detection of charged particles after they have left the plasma. The basic idea is shown in Fig. 5. The initial experiments at NIST on plasmas produced by direct photoionization [2,32,66,67] detected electrons. Experiments exploring the spontaneous evolution of Rydberg atoms into a plasma [64] and dynamics of Rydberg atoms embedded in an ultracold plasma [55,68] have used both ion and electron detection.

For monitoring electrons that escape the plasma during normal evolution, it is essential to not perturb the plasma with large electric fields. However, one needs a field of about $5 \mathrm{mV} / \mathrm{cm}$ to guide the escaping charged particles to the detector. This produces a time-of-flight delay for electrons until they reach

the detector of about $1 \mu \mathrm{s}$. Since electrons move away from the plasma in all directions, it also means that the detection efficiency is not $100 \%$. easily be ramped to much larger values that can quickly dump all charged particles 


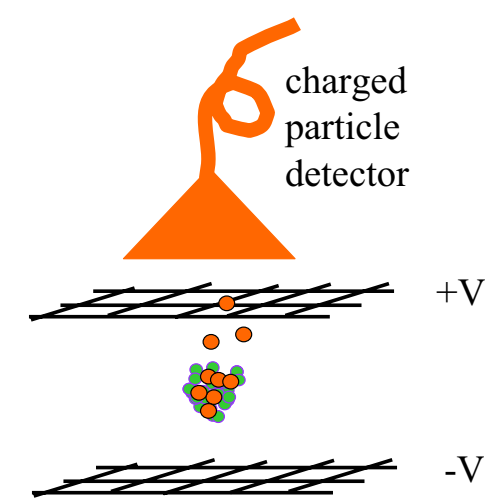

Fig. 5. Charged particle detection diagnostics. When electrons escape from the Coulomb well formed by the ions, electric fields created by potentials on wire-mesh grids direct them to an electron multiplier or microchannel plate. By reversing the electric field, ions can be detected. The grids can also be used to apply oscillating radio frequency fields to excite plasma collective modes.

of a given sign on the detector. With careful design, values well in excess of $100 \mathrm{~V} / \mathrm{cm}$ can be obtained, which are also useful for pulsed field ionization of Rydberg atoms [58].

\subsubsection{Optical probes}

Probing an ultracold plasma through charged particles has some limitations. For example, remote detection of particles tends to only provide information on average properties of the plasma, and cannot resolve variations, such as ion acoustic waves or ion-ion spatial correlations. The time resolution is also limited by the time of flight for electrons to reach the detector.

For detecting electrons, there are currently no options besides charged particle diagnostics. For probing ions, however, optical methods offer a powerful alternative that can provide in situ, non-destructive measurements, with excellent spatial, temporal, and spectral resolution. Absorption imaging [12] has been used to probe ion-ion equilibration and expansion of the plasma during the first few microseconds after photoionization. Fluorescence monitoring has given information on the plasma expansion dynamics [13] from shortly after photoionization to $50 \mu$ s later. Both techniques have great potential to study phenomena such as ion collective modes [69], shock waves [33], recombination, and particle-particle spatial correlations [11].

Absorption imaging is an adaptation of one of the most powerful techniques for studying laser cooled and trapped neutral atoms [52. Implementation of this probe to study ultracold plasmas requires much higher temporal resolution than typically needed for neutral atom experiments because the plasma evolves quickly - in as short as nanoseconds in some cases. It is also essential 


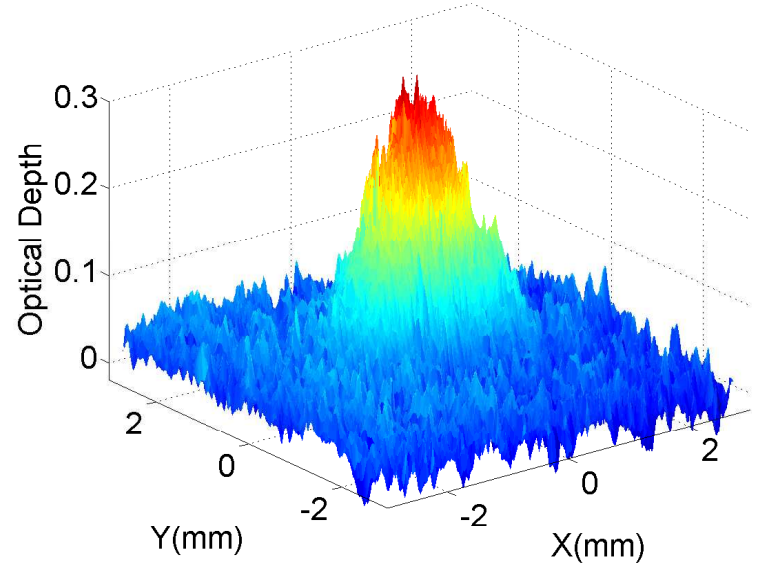

Fig. 6. Optical depth of a strontium ultracold neutral plasma. The delay between the formation of the plasma and image exposure is $85 \mathrm{~ns}$. The plasma contains $7 \times 10^{7}$ ions and the initial peak ion density is $\rho_{0 \mathrm{i}}=2 \times 10^{10} \mathrm{~cm}^{-3}$. Reused with permission from [12. Copyright 2004, American Physical Society.

to perform experiments with an ion whose ground state possesses electricdipole allowed transitions in the optical regime where cameras and lasers are available. Alkaline-earth elements possess this property, which is why they are used in quantum computing and optical frequency standard experiments using trapped ions. Strontium is a good choice for plasma experiments because its laser cooling is well-developed [51] and imaging can be performed using the $\mathrm{Sr}^{+}{ }^{2} S_{1 / 2} \rightarrow{ }^{2} P_{1 / 2}$ transition at $422 \mathrm{~nm}$ (Fig. $3 \mathrm{~B}$ ). Calcium is also a good choice because it has a similar level structure [13,70].

To record an absorption image of the plasma, a collimated laser beam, tuned near resonance with the principal transition in the ions (Fig. $3 \mathrm{~B}$ ), illuminates the plasma and falls on an image-intensified CCD camera. Ions scatter photons out of the laser beam and create a shadow that is recorded by the camera. The optical depth $(O D)$ is defined in terms of the image intensity without the plasma $\left(I_{0}\right)$ and with the plasma present $(I)$ as

$$
O D(x, y)=\ln \left[I_{0}(x, y) / I(x, y)\right]
$$

Figure [6] shows a typical absorption image. By varying the delay $\Delta t$ between the formation of the plasma and image exposure, the time-evolution of the plasma can be studied. The minimum camera exposure gate width for standard intensified CCD cameras is $\sim 10 \mathrm{~ns}$.

Valuable information is provided by the absorption spectrum, which can be recorded by varying the frequency of the probe beam $\nu[12$. For an average spectrum of the entire plasma, the optical depth is integrated over the plane perpendicular to the laser, 


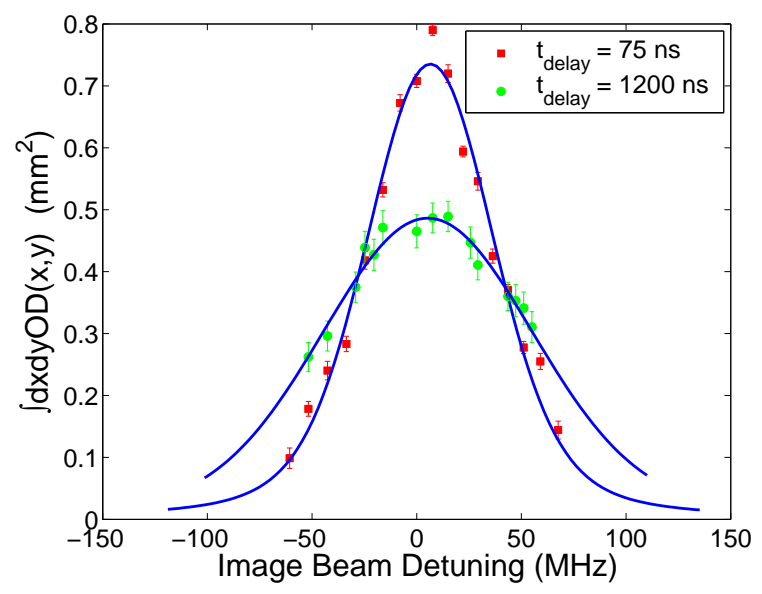

Fig. 7. Absorption spectra of ultracold neutral plasmas (Eq. (91)). The frequency is with respect to a Doppler-free absorption feature in a strontium discharge cell. Both spectra correspond to $T_{e}=56 \mathrm{~K}$ and an initial peak plasma density of $\rho_{0 \mathrm{i}}=2 \times 10^{10} \mathrm{~cm}^{-3}$. Data are fit with Voigt profiles (Eq. (12)). Note the increase in linewidth for longer delay $\Delta t$ (see Eq. (B.4) in appendix B). Reused with permission from [54]. Copyright 2005, Institute of Physics.

$$
S(\nu) \equiv \int d x d y O D(x, y)
$$

In reality the camera pixels are summed, and typical averaged absorption spectra are shown in Fig. 7.

In order to quantitatively interpret the spectra, it is important to account for the inhomogeneous density distribution of the plasma and other effects that will be discussed in subsequent sections. Among them are thermal ion motion, plasma expansion, and the lack of global thermal equilibrium for the ions. Detailed descriptions of the spectral analysis were given in [54] and [71]. Further aspects are also discussed in App. B. The $O D$ can be related to underlying physical parameters according to Beer's law,

$$
O D(x, y)=\int d z \rho_{\mathrm{i}}(x, y, z) \alpha\left[\nu, T_{\mathrm{i}}(r)\right]
$$

where $\rho_{\mathrm{i}}(x, y, z)$ is the ion density, and $\alpha\left[\nu, T_{\mathrm{i}}(r)\right]$ is the ion absorption cross section at the image beam frequency, $\nu$. The absorption cross section is a function of ion kinetic energy, or temperature, due to Doppler broadening. The ion temperature varies with density, so for a spherically symmetric but inhomogenous plasma (e.g., for a gaussian plasma cloud as in Eq. (2) ) $T_{\mathrm{i}}=$ $T_{\mathrm{i}}(r)$ and therefore $\alpha$ varies with position. Equation (10) can be used to relate the spectrum to the density weighted average of the absorption cross section

$$
S(\nu)=\int d^{3} r \rho_{\mathrm{i}}(r) \alpha\left[\nu, T_{\mathrm{i}}(r)\right]
$$


The absorption cross section $\alpha\left[\nu, T_{\mathrm{i}}(r)\right]$ for ions whose motion can be described by a temperature $T_{\mathrm{i}}$ is given by the Voigt profile (see appendix B. Eq. (B.1)). When this expression, generalized to include the effect of the plasma expansion (Eq. (B.3) $)$ is substituted into the expression for the spectrum (Eq. (11)), the result is reasonably complicated. However, to a good approximation [54,71, the density-averaged spectrum can be fit by a single Voigt profile characterized by an effective ion temperature $\left(T_{\mathrm{i}, \text { eff }}\right)$ that describes all the Doppler broadening due to ion motion (For the derivation, see appendix [B]),

$$
S(\nu)=N_{i} \frac{3^{*} \lambda^{2}}{2 \pi} \frac{\gamma_{0}}{\gamma_{\mathrm{eff}}} \int d s \frac{1}{1+4\left(\frac{\nu-s}{\gamma_{\mathrm{eff}} / 2 \pi}\right)^{2}} \frac{1}{\sqrt{2 \pi} \sigma_{D}\left(T_{\mathrm{i}, \mathrm{eff}}\right)} \exp \left[-\frac{\left(s-\nu_{0}\right)^{2}}{2 \sigma_{D}\left(T_{\mathrm{i}, \mathrm{eff}}\right)^{2}}\right]
$$

where $\sigma_{D}(T)=\sqrt{k_{B} T / m_{\mathrm{i}}} / \lambda$ is the Doppler width, and $\gamma_{\text {eff }}=\gamma_{0}+\gamma_{\text {ins }}$ is the effective Lorentzian linewidth due to the natural linewidth $\gamma_{0}$ of the transition and any instrumental linewidth $\gamma_{\text {ins }}$. The center frequency of the transition is $\nu_{0}=c / \lambda$, and the "three-star" symbol [72] is a numerical factor that accounts for the polarization state of the ions and the imaging light.

With reasonable assumptions valid for a wide range of plasma parameters, $T_{\mathrm{i} \text {,eff }}$ can be related to the average ion temperature in the plasma, $T_{\mathrm{i} \text {,ave }}=$ $\int d^{3} r T_{i}(r)$, but the relation varies as the plasma evolves. During the first microsecond after photoionization and while disorder-induced heating (Sec. 4.2.1) takes place, the plasma is not in local thermal equilibrium (LTE). (See Section 4.2.) Strictly speaking, ion temperature is not a well defined concept at in this situation. However, $T_{\mathrm{i} \text {,eff }}$ can always be related to the average ion kinetic energy and the rms ion velocity along the laser beam through $k_{B} T_{\mathrm{i}, \text { eff }}=$ $\frac{2}{3}\left\langle E_{\mathrm{kin}}\right\rangle=m_{i} u_{z, r m s}^{2}$.

Once LTE is established, it is possible to assign an ion temperature reflecting thermal motion, $T_{\mathrm{i}}(r)$, but it varies with position because the plasma lacks global thermal equilibrium [73,71]. The approach to global thermal equilibrium is governed by a heat diffusion equation. This leads to a equilibration time equal to

$$
\tau_{\text {global }} \approx \sigma^{2} / D_{t h}
$$

where the heat diffusion coefficient for a strongly coupled plasma is predicted to be on the order of [74]

$$
D_{\mathrm{th}} \sim a^{2} \omega_{\mathrm{p}, \mathrm{i}} .
$$

This depends on the plasma density through the Wigner-Seitz radius and the ion plasma oscillation frequency. $\tau_{\text {global }}$ is typically greater than $100 \mu \mathrm{s}$, which is longer than experiments or simulations have probed.

The average ion temperature just after establishment of LTE, $T_{\mathrm{i}, \text { ave }}^{L T E}$, is an 
important characteristic scale for thermal ion energy. For times much less than

$$
t_{\mathrm{exp}}=\sqrt{\frac{\sigma_{0}^{2} m_{\mathrm{i}}}{k_{B} T_{\mathrm{e} 0}}} \sqrt{\frac{T_{\mathrm{i}, \mathrm{ave}}^{L T E}}{T_{e}}} \equiv \tau_{\exp } \sqrt{\frac{T_{\mathrm{i}, \mathrm{ave}}^{L T E}}{T_{e}}},
$$

the Doppler shift due to plasma expansion is much less than the Doppler width due to thermal ion motion, and the expansion can be neglected. Here, $\tau_{\exp }$ is the characteristic time scale of plasma expansion which emerges from the kinetic theory of ultracold plasmas. (See Eq. (25) in Section 3.1.1) References [54] and [71] show through numerical simulation that under these conditions, $T_{\mathrm{i}, \mathrm{eff}} \approx C T_{\mathrm{i}, \text { ave }}$, where $C=0.95 \pm 0.05$.

At later times, the expansion is not negligible. But for a broad range of plasma initial conditions, the expansion is quite simple and can be described by assuming quasi-neutrality and a self-similar expansion of the plasma (Section 3.1.1). The effect of the expansion velocity on the Doppler width can then be incorporated into the effective temperature of Eq. (12) as shown in appendix B.

Regardless of the exact spatial dependence of $T_{\mathrm{i}}(\mathbf{r})$, the integral of the spectrum for the entire plasma is proportional to the number of ions $N_{\mathrm{i}}$ in the plasma and the natural linewidth of the transition. This provides an absolute calibration of the signal through

$$
\int d \nu S(\nu)=\int d \nu d^{3} r \rho_{\mathrm{i}}(\mathbf{r}) \alpha\left[\nu, T_{\mathrm{i}}(\mathbf{r})\right]=\frac{3^{*} \lambda^{2} N_{\mathrm{i}} \gamma_{0}}{8 \pi}
$$

If Eq. (9) is modified so that the $O D$ is integrated over a subsection of the plasma image, then $T_{\mathrm{i} \text {,eff }}$ describes ion motion in the region bounded by the integration limits and extending along the propagation direction of the imaging laser. The integral over the spectrum is then proportional to the number of ions in that region. Analysis of spectra in annular regions was described in [28,71] and proved very useful for studying kinetic energy oscillations (Section 4.2).

In fluorescence experiments, which have been performed with calcium [13,70], fluorescence from a cylindrical region formed by the intersection of the plasma with a tightly focused laser beam is collected on a photomultiplier tube. (See Fig. 8.) This approach has the advantage of recording the full time evolution of the system with each photoionizing pulse, but repeated scans must be taken with the laser at different positions in order to map the spatial distribution.

When the only Doppler-broadening of the optical transition arises from ther- 


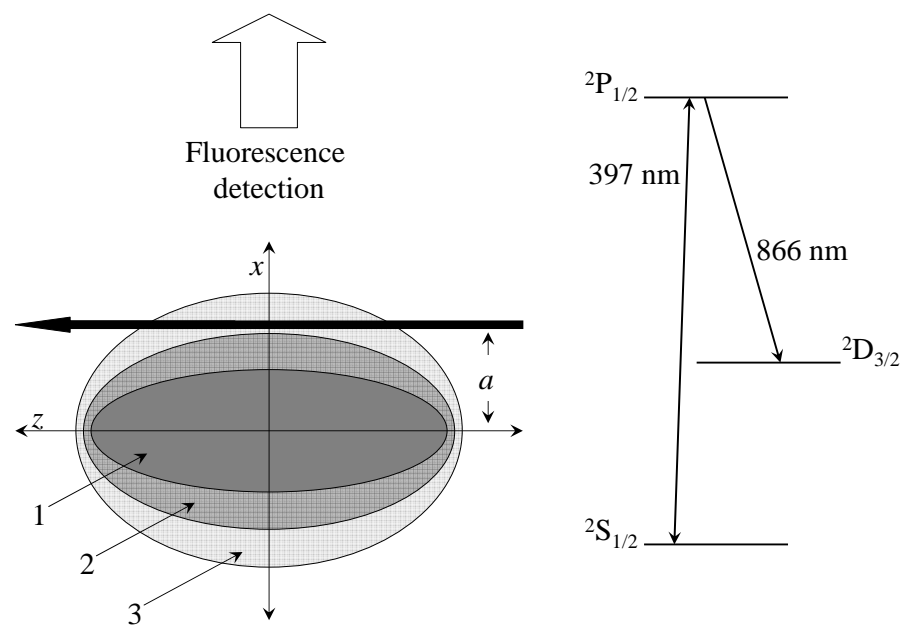

Fig. 8. Fluorescence detection in an expanding ultracold neutral calcium plasma. Left - The plasma is elongated along z. Regions 1-3 occur sequentially in time and show how the plasma expands predominantly in the $\mathrm{x}-\mathrm{y}$ plane. The laser excitation probe propagates parallel to the $\mathrm{z}$ axis. Right - Electronic levels of the calcium ion important for the experiment. Reused with permission from [70]. Copyright 2005, American Institute of Physics.

mal motion of the ions, and the ion velocity distribution along the laser beam can be written as a Maxwellian of the form $P\left(v_{z}\right)=\exp \left(-v_{z}^{2} / 2 v_{\mathrm{th}}^{2}\right) / \sqrt{2 \pi} v_{\mathrm{th}}$, the amplitude of the fluorescence signal on resonance is related to the thermal velocity, $v_{\text {th }}$, through [13]

$$
S \propto \frac{1}{v_{\mathrm{th}}} \exp \left(-\frac{b^{2}}{2 v_{\mathrm{th}}^{2}}\right) \operatorname{erfc}\left(\frac{b}{\sqrt{2} v_{\mathrm{th}}}\right),
$$

where $b=\gamma \lambda / 2$ is the velocity that corresponds to a Doppler shift equal to the natural linewidth of the transition, $\gamma$. If the temperature of the ions is known at some time, the signal can be calibrated and the evolution of the ion temperature can be extracted [13. When Doppler broadening due to the expansion velocity of the ions becomes important, an analogous expression can be derived that allows one to obtain information on the expansion dynamics [70].

\section{Theoretical Description}

In the last decades, a variety of methods have been developed to describe equilibrium states [75,76,77,78,79], relaxation processes [80,81, transport properties [82,83], and atomic processes [84,85,86,87] of plasmas. These methods 
cover a wide parameter space, ranging from cold, low-density plasmas in gasdischarges to warm dense matter systems. A typical ultracold plasma behaves classically, which facilitates its description. Indeed, the low particle density in an ultracold plasma implies a Brückner parameter $r_{\mathrm{s}}=\left(3 / 4 \pi \rho_{\mathrm{e}}\right)^{1 / 3} / a_{0} \gg 1$ and an electronic Fermi energy $E_{\mathrm{F}} \ll k_{\mathrm{B}} T_{\mathrm{e}}$. Hence, the values of both of these parameters justify a classical treatment of the free charges. Moreover, due to the low electron temperatures, recombination forms atoms in highly excited states, such that electron-atom collisions [44] and radiative transitions [88] can be described classically as well. One might be tempted to conclude that a macroscopic treatment based on kinetic theory incorporating some collision phenomena may suffice to describe ultracold plasmas.

However, as it has turned out this is not true. Even a classical description of ultracold neutral plasmas is far from trivial and poses several challenges. First of all (and this is of course one reason why ultracold plasmas are attractive) the ionic plasma component may be strongly coupled, rendering common kinetic theories for weakly coupled plasmas inapplicable. Strongly coupled problems are usually tackled numerically with a molecular-dynamics (MD) approach. Due to the open boundary conditions and long evolution times of ultracold plasmas, however, a full MD description of the plasma dynamics constitutes an intractable computational task currently. Moreover, the highly non-equilibrium plasma dynamics exhibits different relaxation processes on very different time scales, which all have to be appropriately taken into account for a reliable and realistic understanding of an ultracold plasma.

Hence, one may ask if it is possible at all to describe ultracold plasmas on a macroscopic level based on kinetic and hydrodynamic formulations. This is indeed possible to some extent, as we will detail in Section 3.1. Starting from a collisionless description of the plasma, additional effects such as inelastic collisions and strong ion correlations are successively incorporated into the model. More sophisticated, but also technically more demanding, approaches, which describe the plasma dynamics on a microscopic level, are introduced in Section 3.2. They can be used and are necessary to assess the validity and limitations of the macroscopic descriptions.

\subsection{Macroscopic Approaches}

\subsubsection{Collisionless plasma dynamics}

If we assume the Coulomb coupling parameter of both the electrons and the ions to be much less than unity and neglect any collisional processes in the plasma, the dynamical equations describing the one-particle phase space den- 
sity $f_{\alpha}\left(\mathbf{r}_{\alpha}, \mathbf{v}_{\alpha}\right)$ [89] simplify to the well known Vlasov equations

$$
\frac{\partial f_{\alpha}}{\partial t}+\mathbf{v}_{\alpha} \frac{\partial f_{\alpha}}{\partial \mathbf{r}_{\alpha}}-m_{\alpha}^{-1} \frac{\partial f_{\alpha}}{\partial \mathbf{v}_{\alpha}} q_{\alpha} \frac{\partial \varphi\left(\mathbf{r}_{\alpha}\right)}{\partial \mathbf{r}_{\alpha}}=0
$$

Here, $\alpha=\mathrm{e}$, i for electrons and ions, respectively $11, m_{\alpha}$ and $q_{\alpha}$ are the mass and charge of species $\alpha$, and $\varphi$ is the total mean-field potential determined by the Poisson equation

$$
\Delta \varphi=\frac{e}{\varepsilon_{0}}\left(\rho_{\mathrm{e}}-\rho_{\mathrm{i}}\right),
$$

with $\rho_{\alpha}=\int f_{\alpha} d \mathbf{v}_{\alpha}$. As a paradigmatic problem of plasma physics, the collisionless plasma expansion into vacuum has been considered for a long time [90,91 and solutions of Eqs. (18) have been investigated in a number of recent publications [92,93]. Ultracold plasmas for the first time allow for a combined theoretical and experimental study of this problem. Clearly, for the present situation of potentially strongly coupled plasmas, the Vlasov equations alone will not suffice. But they will serve as the basis of our hydrodynamic approach. A suitable approximate treatment of correlation effects will subsequently be added step by step, to clarify the influence of correlations on the expansion dynamics.

Due to its nonlinearity a general, closed-form analytical solution of Eqs. (18) cannot be given. Here we will employ the additional assumption of quasineutrality [91, under which a large set of self-similar solutions exists. Of particular interest in the context of ultracold plasmas is the case of a Gaussian phase space density (see Eq.(22)), discussed in [92], since this is the typical initial shape of the plasma created in a magneto-optical trap. For a spherically symmetric plasma it describes a local equilibrium in velocity space, which holds for arbitrarily long times of the plasma evolution. We will use a further approximation, enforcing a local equilibrium for the electrons (see Eq.(20)), in order to treat more general cases of strongly coupled or non-symmetric plasmas as discussed below. Due to the very small mass ratio $m_{\mathrm{e}} / m_{\mathrm{i}}$, the timescale for equilibration of the electrons is much smaller than the timescale for motion of the ions. Exploiting this fact, one may safely use an adiabatic approximation for the electrons, assuming instant equilibration, such that the electronic phase space density is given by a quasistationary distribution

$$
\begin{aligned}
f_{\mathrm{e}}(\mathbf{r}, \mathbf{v}, t) & =f_{\mathrm{e}}^{(\mathrm{qs})}\left(\mathbf{r}, \mathbf{v}, T_{\mathrm{e}}(t)\right) \\
& =\rho_{\mathrm{e}}(\mathbf{r}, t) \phi_{\mathrm{e}}^{(\mathrm{qs})}\left(\mathbf{v}, T_{\mathrm{e}}(t)\right) \propto \rho_{\mathrm{e}}(\mathbf{r}, t) \exp \left(-\frac{m_{\mathrm{e}} v^{2}}{2 k_{\mathrm{B}} T_{\mathrm{e}}(t)}\right),
\end{aligned}
$$

where the time dependence of the velocity distribution function $\phi_{\mathrm{e}}^{(\mathrm{qs})}$ is only implicit in the time dependence of $T_{\mathrm{e}}$.

$\overline{1}$ Here and in the remainder of this section, greek indices denote particle species while roman indices label individual particles of a given species. 
Substituting this expression into the electronic Vlasov equation (18) together with the quasineutrality condition $\left(\rho_{\mathrm{e}}(\mathbf{r}) \approx \rho_{\mathrm{i}}(\mathbf{r})\right)$ allows one to express the mean-field potential in terms of the ion density

$$
e \frac{\partial \varphi}{\partial \mathbf{r}}=k_{\mathrm{B}} T_{\mathrm{e}} \rho_{\mathrm{e}}^{-1} \frac{\partial \rho_{\mathrm{e}}}{\partial \mathbf{r}} \approx k_{\mathrm{B}} T_{\mathrm{e}} \rho_{\mathrm{i}}^{-1} \frac{\partial \rho_{\mathrm{i}}}{\partial \mathbf{r}}
$$

which together with the ionic Vlasov equation (18) yields a closed set of equations for the ionic phase space density $f_{\mathrm{i}}$. Note, that the condition $\rho_{\mathrm{e}}(\mathbf{r}) \approx \rho_{\mathrm{i}}(\mathbf{r})$ does not imply the space charge potential to be zero. Rather, it replaces the Poisson equation (19) by Eq. (21). In appendix C we provide a more detailed account of this fact, which may seem somewhat contradictorily and is rarely discussed in the literature.

For a Gaussian spatial plasma density, Eq. (21) implies a linear force such that the simple ansatz

$$
f_{\mathrm{i}} \propto \exp \left(-\sum_{k} \frac{r_{k}^{2}}{2 \sigma_{k}^{2}}\right) \exp \left(-\sum_{k} \frac{m_{\mathrm{i}}\left(v_{k}-\gamma_{k} r_{k}\right)^{2}}{2 k_{B} T_{\mathrm{i}, k}}\right)
$$

provides a selfsimilar solution of the ionic kinetic equation. Here the index $k=x, y, z$ labels the (cartesian) components, $\sigma$ is the rms-radius of the Gaussian spatial distribution, and $\gamma$ is a parameter determining the local mean of the velocity distribution, i.e. the hydrodynamic ion velocity. Coincidentally, the Gaussian form (22) corresponds to the initial distribution of ions, which reflects the Gaussian density profile and zero mean velocity (i.e. $\gamma=0$ ) of atoms in the magneto-optical trap. Hence, within the collisionless quasineutral approximation, the expanding plasma retains its shape for all times, and its evolution can be parameterized by the macroscopic parameters $\sigma_{k}, \gamma_{k}, T_{\mathrm{i}, k}$ and $T_{\mathrm{e}}$ only.

Considering a spherically symmetric plasma of Gaussian form as in Eq. (2) and substituting the ansatz Eq. (22) into Eq. (18) yields the following set of equations for the plasma parameters

$$
\begin{aligned}
\frac{\partial}{\partial t} \sigma^{2} & =2 \gamma \sigma^{2} \\
\frac{\partial}{\partial t} \gamma & =\frac{\left(k_{\mathrm{B}} T_{\mathrm{e}}+k_{\mathrm{B}} T_{\mathrm{i}}\right)}{m_{\mathrm{i}} \sigma^{2}}-\gamma^{2}, \\
\frac{\partial}{\partial t}\left(k_{\mathrm{B}} T_{\mathrm{i}}\right) & =-2 \gamma k_{\mathrm{B}} T_{\mathrm{i}}, \\
\frac{\partial}{\partial t}\left(k_{\mathrm{B}} T_{\mathrm{e}}\right) & =-2 \gamma k_{\mathrm{B}} T_{\mathrm{e}} .
\end{aligned}
$$

In addition to the conserved total energy $E_{\mathrm{tot}}=\frac{3}{2} N_{\mathrm{i}} k_{\mathrm{B}}\left(T_{\mathrm{e}}+T_{\mathrm{i}}\right)+\frac{3}{2} N_{\mathrm{i}} m_{\mathrm{i}} \gamma^{2} \sigma^{2}$ Eqs. 23 possess two integrals of motion $\sigma^{2} T_{\mathrm{e}}=$ const. and $\sigma^{2} T_{\mathrm{i}}=$ const., re- 
flecting the adiabatic cooling of both the electrons and the ions during the plasma expansion. Using these constants of motion, a simple analytical solution of Eqs. (23) describing the collisionless plasma expansion can be found

$$
\begin{aligned}
\sigma^{2}(t) & =\sigma^{2}(0)\left(1+t^{2} / \tau_{\exp }^{2}\right) \\
\gamma(t) & =\frac{t / \tau_{\exp }^{2}}{1+t^{2} / \tau_{\exp }^{2}}, \\
T_{\mathrm{i}}(t) & =\frac{T_{\mathrm{i}}(0)}{1+t^{2} / \tau_{\exp }^{2}}, \\
T_{\mathrm{e}}(t) & =\frac{T_{\mathrm{e}}(0)}{1+t^{2} / \tau_{\exp }^{2}}
\end{aligned}
$$

where the characteristic plasma expansion time is given by

$$
\tau_{\exp }=\sqrt{\frac{m_{\mathrm{i}} \sigma^{2}(0)}{k_{\mathrm{B}} T_{\mathrm{e}}(0)}},
$$

and the expansion velocity is

$$
\mathbf{u}(\mathbf{r}, t)=\gamma(t) \mathbf{r}
$$

Measurements of the average density dynamics for ultracold plasmas are in very good agreement with these simple relations for high initial electron temperature $\left(T_{\mathrm{e}}(0)>70 \mathrm{~K}\right)$ 32]. However, the same experiments have revealed significant deviations from this average density dynamics for lower electron temperature. As discussed in [42], they mainly arise from inelastic collisions, i.e., from the formation of Rydberg atoms during the plasma expansion. This is one consequence of collisions in the plasma which we will discuss next.

\subsubsection{Collisional processes}

There are two types of collisional processes in plasmas: elastic collisions, which do not affect the total system temperature $T_{\mathrm{e}}+T_{\mathrm{i}}$, and inelastic collisions, which may drastically change the temperature of the electrons. On the shortest timescale of several ns, elastic electron-electron collisions lead to an equilibration of the electron velocities, giving rise to the electronic temperature of the plasma. Implicitly, we have already accounted for this process in the previous section within the adiabatic approximation Eq. (20), which assumes an instantaneous relaxation towards a Maxwellian electron velocity distribution. This assumption is well justified since the timescale for electron-electron collisions is by far the shortest one for a typical ultracold plasma. The ion velocity distribution, on the other hand, has the initial Maxwellian form of 
the atomic velocity distribution. Hence, the ion-ion collision integral vanishes and does not change the functional velocity dependence of the ion distribution as described by Eq. (22).

While electron-electron and ion-ion collisions lead to an individual electron and ion temperature, respectively, electron-ion collisions tend to equilibrate the ion and the electron temperature. However, elastic electron-ion collisions may safely be neglected for the description of a freely expanding plasma 2 , since the corresponding ion heating rate [60] is much less than the rate of the initial disorder induced ion heating to be discussed in Section 4.2.1. During later times, the expansion leads to an adiabatic cooling of the ions, but also to a reduction of the collisional heating rate. Hence, elastic electron-ion collisions remain negligible at all times [94].

This is not the case, however, for inelastic electron-ion collisions due to the low temperature of the electrons, which leads to efficient recombination into bound Rydberg states. The formation of bound states during the evolution of the plasma can be described most accurately within a classical molecular dynamics treatment taking into account all electron-ion interactions [37,38. However, as discussed above, such an approach is limited to short times due to the fact that the small timescale on which the electronic motion takes place has to be resolved. Within the present macroscopic approach the problem is simplified significantly by adopting a chemical picture, i.e., by introducing neutral atoms as an additional particle species. Naturally, this transition is accomplished by splitting the electron-ion two-particle distribution function $f_{\mathrm{ei}}$ into a free and a bound part [84, where the bound part is identified with the distribution function $f_{\mathrm{a}}$ of the atoms. The evolution equation for the atom distribution function then involves an integral over the three-particle distribution function describing correlations between pairs of electrons and an ion, which accounts for inelastic collisions, i.e. recombination, ionization and electron impact (de)-excitation. Without going into detail, the corresponding inelastic processes can be identified as three-body recombination, electron-impact ionization, and electron impact excitation or deexcitation. Consequently, the electron-ion kinetic equation (18) splits into separate equations for free electrons, ions, and atoms. Neglecting atom-atom interactions and still retaining the Vlasov approximation, the kinetic equations for ions and atoms read

$$
\begin{aligned}
\frac{\partial f_{\mathrm{i}}}{\partial t}+\mathbf{v}_{\mathrm{i}} \frac{\partial f_{\mathrm{i}}}{\partial \mathbf{r}_{\mathrm{i}}}-e m_{\mathrm{i}} \frac{\partial f_{\mathrm{i}}}{\partial \mathbf{v}_{\mathrm{i}}} \frac{\partial \varphi}{\partial \mathbf{r}_{\mathrm{i}}} & =J_{\mathrm{ei}}^{(3)}, \\
\frac{\partial f_{\mathrm{a}}}{\partial t}+\mathbf{v}_{\mathrm{a}} \frac{\partial f_{\mathrm{a}}}{\partial \mathbf{r}_{\mathrm{a}}} & =J_{\mathrm{ae}} .
\end{aligned}
$$

2 Note that the situation is drastically different in laser-cooled plasmas considered in Section 5.1 .1 
The collision terms on the right-hand sides of Eq. (27) are given by

$$
J_{\mathrm{ei}}^{(3)}=\sum_{n} K_{\text {ion }}\left(n, \rho_{\mathrm{e}}\left(\mathbf{r}_{\mathrm{i}}\right), T_{\mathrm{e}}\right) f_{\mathrm{a}}\left(n, \mathbf{p}_{\mathrm{i}}, \mathbf{r}_{\mathrm{i}}\right)-\sum_{n} K_{\mathrm{tbr}}\left(n, \rho_{\mathrm{e}}\left(\mathbf{r}_{\mathrm{i}}\right), T_{\mathrm{e}}\right) f_{\mathrm{i}}\left(\mathbf{p}_{\mathrm{i}}, \mathbf{r}_{\mathrm{i}}\right)
$$

and

$$
\begin{aligned}
J_{\mathrm{ae}}= & \sum_{p}\left[K_{\mathrm{bb}}\left(p, n, \rho_{\mathrm{e}}\left(\mathbf{r}_{\mathrm{a}}\right), T_{\mathrm{e}}\right) f_{\mathrm{a}}\left(p, \mathbf{p}_{\mathrm{a}}, \mathbf{r}_{\mathrm{a}}\right)-K_{\mathrm{bb}}\left(n, p, \rho_{\mathrm{e}}\left(\mathbf{r}_{\mathrm{a}}\right), T_{\mathrm{e}}\right) f_{\mathrm{a}}\left(n, \mathbf{p}_{\mathrm{a}}, \mathbf{r}_{\mathrm{a}}\right)\right] \\
& +K_{\mathrm{tbr}}\left(n, \rho_{\mathrm{e}}\left(\mathbf{r}_{\mathrm{a}}\right), T_{\mathrm{e}}\right) f_{\mathrm{i}}\left(\mathbf{p}_{\mathrm{a}}, \mathbf{r}_{\mathrm{a}}\right)-K_{\mathrm{ion}}\left(n, \rho_{\mathrm{e}}\left(\mathbf{r}_{\mathrm{a}}\right), T_{\mathrm{e}}\right) f_{\mathrm{a}}\left(n, \mathbf{p}_{\mathrm{a}}, \mathbf{r}_{\mathrm{a}}\right)
\end{aligned}
$$

where the rate coefficients $K_{\mathrm{tbr}}, K_{\mathrm{ion}}$ and $K_{\mathrm{bb}}$ for three-body recombination, electron impact ionization and electron impact induced bound-bound transitions depend on the atomic principal quantum numbers $n$ and $p$, the electronic density $\rho_{\mathrm{e}}$, and the temperature $T_{\mathrm{e}}$. Since these coefficients describe threebody processes, no exact analytical expressions for the rates are known. For the highly excited Rydberg states in ultracold plasmas an accurate description is expected from the rate coefficients of [44], which have been obtained by fitting analytical functions to the results of classical trajectory Monte Carlo calculations. Since the collision integrals Eqs. (28) and (29) introduce a nonlinearity in the spatial particle densities, an exact selfsimilar solution of the corresponding kinetic equations can no longer be found. Nevertheless, we assume the phase space distribution to be the same for atoms and for ions and to still be given by Eq. (22), which was shown to yield a good description of freely expanding ultracold plasmas by comparison with more sophisticated calculations [39]. Based on this approximation and from the moments of the distribution function

$$
\begin{aligned}
& \left\langle r^{2}\right\rangle=3 \sigma^{2}=N_{\mathrm{i}}^{-1} \int r^{2} f_{\mathrm{i}}\left(\mathbf{r}_{\mathrm{i}}, \mathbf{v}_{\mathrm{i}}, t\right) d \mathbf{r}_{\mathrm{i}} d \mathbf{v}_{\mathrm{i}} \\
& \langle\mathbf{v r}\rangle=3 \gamma \sigma^{2}=N_{\mathrm{i}}^{-1} \int \mathbf{v}_{\mathrm{i}} \mathbf{r}_{\mathrm{i}} f_{\mathrm{i}}\left(\mathbf{r}_{\mathrm{i}}, \mathbf{v}_{\mathrm{i}}, t\right) d \mathbf{r}_{\mathrm{i}} d \mathbf{v}_{\mathrm{i}} \\
& \left\langle v^{2}\right\rangle=3\left(\frac{k_{B} T_{\mathrm{i}}}{m_{\mathrm{i}}}+\gamma^{2} \sigma^{2}\right)=N_{\mathrm{i}}^{-1} \int v_{\mathrm{i}}^{2} f_{\mathrm{i}}\left(\mathbf{r}_{\mathrm{i}}, \mathbf{v}_{\mathrm{i}}, t\right) d \mathbf{r}_{\mathrm{i}} d \mathbf{v}_{\mathrm{i}} .
\end{aligned}
$$

we finally obtain simple evolution equations for the parameters of the plasma 


$$
\begin{aligned}
\frac{\partial}{\partial t} \sigma^{2} & =2 \gamma \sigma^{2} \\
\frac{\partial}{\partial t} \gamma= & \frac{N_{\mathrm{i}}}{N_{\mathrm{i}}+N_{\mathrm{a}}} \frac{k_{\mathrm{B}} T_{\mathrm{e}}+k_{\mathrm{B}} T_{\mathrm{i}}}{m_{\mathrm{i}} \sigma^{2}}-\gamma^{2} \\
\frac{\partial}{\partial t}\left(k_{\mathrm{B}} T_{\mathrm{i}}\right)= & -2 \gamma k_{\mathrm{B}} T_{\mathrm{i}} \\
\frac{\partial}{\partial t} \mathcal{N}_{\mathrm{a}}(n)= & \sum_{p \neq n}\left[R_{\mathrm{bb}}(p, n) \mathcal{N}_{\mathrm{a}}(p)-R_{\mathrm{bb}}(n, p) \mathcal{N}_{\mathrm{a}}(n)\right] \\
& +R_{\mathrm{tbr}}(n) N_{\mathrm{i}}-R_{\mathrm{ion}}(n) \mathcal{N}_{\mathrm{a}}(n) \\
E_{\text {tot }}= & \frac{3}{2} N_{\mathrm{i}}\left[k_{\mathrm{B}} T_{\mathrm{e}}+k_{\mathrm{B}} T_{\mathrm{i}}+m_{\mathrm{i}} \gamma^{2} \sigma^{2}\right]-\sum_{n} \mathcal{N}_{\mathrm{a}}(n) \frac{\mathcal{R}}{n^{2}}=\text { const. },
\end{aligned}
$$

where $\mathcal{R}$ is the Rydberg constant which has the value $\mathcal{R}=13.6 \mathrm{eV}$ in electron Volts and $\mathcal{R}=2.18 \times 10^{-18} \mathrm{~J}$ in SI-units. The number of atoms with principal quantum number $n$ is denoted by $\mathcal{N}_{\mathrm{a}}(n)$, where $\sum_{n} \mathcal{N}_{\mathrm{a}}(n)=N_{\mathrm{a}}$, and the density averaged collision rates are defined as

$$
\begin{aligned}
R_{\mathrm{bb}}(n, p) & =N_{\mathrm{i}}^{-1} \int \rho_{\mathrm{i}}(\mathbf{r}) K_{\mathrm{bb}}\left(n, p, \rho_{\mathrm{i}}\left(\mathbf{r}, T_{\mathrm{e}}\right)\right) d \mathbf{r} \\
R_{\mathrm{ion}}(n) & =N_{\mathrm{i}}^{-1} \int \rho_{\mathrm{i}}(\mathbf{r}) K_{\mathrm{ion}}\left(n, \rho_{\mathrm{i}}\left(\mathbf{r}, T_{\mathrm{e}}\right)\right) d \mathbf{r} \\
R_{\mathrm{tbr}}(n) & =N_{\mathrm{i}}^{-1} \int \rho_{\mathrm{i}}(\mathbf{r}) K_{\mathrm{tbr}}\left(n, \rho_{\mathrm{i}}\left(\mathbf{r}, T_{\mathrm{e}}\right)\right) d \mathbf{r} .
\end{aligned}
$$

As can be seen from the energy relation Eq. (31e), inelastic collisions not only change the total number of atoms and ions, i.e., the degree of ionization of the plasma, but also affect the temperature of the electronic component. Threebody recombination heats the electron plasma, since one electron carries away the excess energy gained in the recombination of another electron with an ion. Subsequent de-exciting electron-Rydberg atom collisions further heat up the plasma, electron impact ionization and excitation tend to cool the electrons.

The total three-body recombination rate has a strong temperature dependence and scales as $\sum_{n} R_{\mathrm{tbr}}(n) \propto T_{\mathrm{e}}^{-9 / 2}$. Hence, for very high initial electron temperatures inelastic collisions are of minor importance and Eqs. (31) reduce to Eqs. (23)), such that the plasma expansion is well described by the simple relations Eqs. (24). On the other hand, at low initial $T_{\mathrm{e}}$, when the electronic Coulomb coupling parameter approaches unity, three-body recombination and subsequent electron-atom collisions quickly heat up the electron gas, strongly decreasing $\Gamma_{\mathrm{e}}$ [95]. This justifies our assumption of an ideal electron gas, inherent in the kinetic description. The ionic temperature, on the other hand, remains practically unaffected by inelastic collisions. Hence, ionic correlation effects may not necessarily be negligible, and will be accounted for in the next section. 


\subsubsection{Strongly coupled ions}

Kinetic theories of non-ideal plasmas are commonly based on a small- $\Gamma$ expansion. For ultracold plasmas such approaches are clearly not applicable, since the ion component can exhibit Coulomb coupling parameters on the order of or even much larger than unity. Alternatively, one can combine a hydrodynamical treatment of the expansion dynamics with numerical results for homogeneous strongly coupled plasmas [39]. The starting point is the kinetic equation for the ions, which accounts exactly for ion-ion correlations,

$$
\left(\frac{\partial}{\partial t}+\mathbf{v}_{\mathrm{i}} \frac{\partial}{\partial \mathbf{r}_{\mathrm{i}}}-\frac{e}{m_{\mathrm{i}}} \frac{\partial \varphi}{\partial \mathbf{r}_{\mathrm{i}}} \frac{\partial}{\partial \mathbf{v}_{\mathrm{i}}}\right) f_{\mathrm{i}}\left(\mathbf{r}_{\mathrm{i}}, \mathbf{v}_{\mathrm{i}}, t\right)=\frac{\partial}{\partial \mathbf{p}_{\mathrm{i}}} \int\left(\frac{\partial V_{\mathrm{ii}}}{\partial \mathbf{r}_{\mathrm{i}}}\right) c_{\mathrm{ii}}\left(\mathbf{r}_{\mathrm{i}}, \mathbf{v}_{\mathrm{i}}, \mathbf{r}_{\mathrm{i}^{\prime}}, \mathbf{v}_{\mathrm{i}^{\prime}}, t\right) d \mathbf{r}_{\mathrm{i}^{\prime}} d \mathbf{v}_{\mathrm{i}^{\prime}},
$$

where $c_{\mathrm{ii}}$ is the ion-ion correlation function. To simplify notation we neglect inelastic collisions whose contributions can straightforwardly be added, as described in the previous section.

As before, from Eq. (33) we derive the time evolution of the moments of $f_{\mathrm{i}}$ up to second order, which together with the ansatz Eq. (22) yields

$$
\begin{aligned}
\frac{\partial}{\partial t} \sigma^{2} & =2 \gamma \sigma^{2} \\
\frac{3}{2} m_{\mathrm{i}} \frac{\partial}{\partial t} \gamma \sigma^{2}= & \frac{3}{2} m_{\mathrm{i}} \gamma^{2} \sigma^{2}+\frac{3}{2} k_{\mathrm{B}} T_{\mathrm{i}}+\frac{3}{2} k_{\mathrm{B}} T_{\mathrm{e}} \\
& +\frac{1}{2 N_{\mathrm{i}}} \int \rho_{\mathrm{i}}\left(\mathbf{r}_{\mathrm{i}}\right) \mathbf{r}_{\mathrm{i}} \mathbf{F}_{\mathrm{ii}}\left(\mathbf{r}_{\mathrm{i}}\right) d \mathbf{r}_{\mathrm{i}} \\
\frac{3}{2} \frac{\partial}{\partial t}\left(k_{\mathrm{B}} T_{\mathrm{i}}+m_{\mathrm{i}} \gamma^{2} \sigma^{2}\right)= & \gamma k_{\mathrm{B}} T_{\mathrm{e}}-\frac{\partial U_{\mathrm{ii}}}{\partial t} .
\end{aligned}
$$

The correlation pressure force $\mathbf{F}_{\mathrm{ii}}$ and the correlation energy $U_{\mathrm{ii}}$ are obtained from

$$
\mathbf{F}_{\mathrm{ii}}=-\frac{1}{3}\left(\frac{u_{\mathrm{ii}}}{\rho_{\mathrm{i}}}+\frac{\partial u_{\mathrm{ii}}}{\partial \rho_{\mathrm{i}}}\right) \frac{\partial \rho_{\mathrm{i}}}{\partial \mathbf{r}_{\mathrm{i}}}
$$

and

$$
U_{\mathrm{ii}}=\frac{1}{N_{\mathrm{i}}} \int \rho_{\mathrm{i}}\left(\mathbf{r}_{\mathrm{i}}\right) u_{\mathrm{ii}}\left(\mathbf{r}_{\mathrm{i}}\right) d \mathbf{r}_{\mathrm{i}},
$$

respectively, where

$$
u_{\mathrm{ii}}=\frac{e^{2}}{2} \rho_{\mathrm{i}}\left(\mathbf{r}_{\mathrm{i}}\right) \int \frac{g_{\mathrm{ii}}\left(y, \rho_{\mathrm{i}}\left(\mathbf{r}_{\mathrm{i}}\right)\right)}{y} d \mathbf{y}
$$

is the mean correlation energy per particle and $g_{\mathrm{ii}}\left(r, \rho\left(\mathbf{r}_{\mathrm{i}}\right)\right)$ is the spatial pair correlation function of a homogeneous plasma of density $\rho\left(\mathbf{r}_{\mathbf{i}}\right)$. Using

$$
\frac{1}{N_{\mathrm{i}}} \int \rho_{\mathrm{i}}(\mathbf{r}) \mathbf{r} \mathbf{F}_{\mathrm{ii}}(\mathbf{r}) d \mathbf{r}=-\frac{1}{N_{\mathrm{i}}} \int u_{\mathrm{ii}} \rho_{\mathrm{i}} d \mathbf{r}=-U_{\mathrm{ii}},
$$


Eqs. (34) are rewritten as

$$
\begin{aligned}
\frac{\partial \sigma^{2}}{\partial t} & =2 \gamma \sigma^{2} \\
\frac{\partial \gamma}{\partial t} & =\frac{k_{\mathrm{B}} T_{\mathrm{e}}+k_{\mathrm{B}} T_{\mathrm{i}}+\frac{1}{3} U_{\mathrm{ii}}}{m_{\mathrm{i}} \sigma^{2}}-\gamma^{2} \\
\frac{\partial k_{\mathrm{B}} T_{\mathrm{i}}}{\partial t} & =-2 \gamma k_{\mathrm{B}} T_{\mathrm{i}}-\frac{2}{3} \gamma U_{\mathrm{ii}}-\frac{2}{3} \frac{\partial U_{\mathrm{ii}}}{\partial t} \\
\frac{\partial k_{\mathrm{B}} T_{\mathrm{e}}}{\partial t} & =-2 \gamma k_{\mathrm{B}} T_{\mathrm{e}},
\end{aligned}
$$

where the last equation follows from energy conservation,

$$
E_{\text {tot }}=N_{\mathrm{i}} \frac{m_{\mathrm{i}}}{2}\left\langle v^{2}\right\rangle+\frac{3}{2} N_{\mathrm{i}} k_{\mathrm{B}} T_{\mathrm{e}}+N_{\mathrm{i}} U_{\mathrm{ii}}
$$

While the description of the influence of ionic correlations on the plasma dynamics via the single macroscopic quantity $U_{\text {ii }}$ constitutes a significant simplification of the problem, the system of eqs. (39) is still not complete since it does not contain an equation for the time evolution of $U_{\text {ii }}$. An accurate description of the evolution of $U_{\mathrm{ii}}$ on a kinetic level is rather complicated, and beyond the capabilities of the simple approach pursued here. A useful and reasonable approximation for the evolution of $U_{\mathrm{ii}}$ is the so-called correlation-time approximation 96]

$$
\frac{\partial U_{\mathrm{ii}}}{\partial t} \approx-\frac{U_{\mathrm{ii}}-U_{\mathrm{ii}}^{(\mathrm{eq})}}{\tau_{\mathrm{corr}}}
$$

where $\tau_{\text {corr }}=\sqrt{m_{\mathrm{i}} \varepsilon_{0} /\left(e^{2} \rho_{\mathrm{i}}\right)}=\omega_{\mathrm{p}, \mathrm{i}}^{-1}$ is the timescale on which pair correlations relax towards their equilibrium value,

$$
U_{\mathrm{ii}}^{(\mathrm{eq})}=\int \rho_{\mathrm{i}}(\mathbf{r}) u_{\mathrm{ii}}^{(\mathrm{eq})}(\mathbf{r}) d \mathbf{r},
$$

and $u_{\mathrm{ii}}^{(\mathrm{eq})}$ is the correlation energy of a homogeneous one-component plasma (OCP) of density $\rho_{\mathrm{i}}(\mathbf{r})$ in thermodynamical equilibrium [97]. It is a special property of the Coulomb potential that the temperature scaled correlation energy is uniquely determined by the Coulomb coupling parameter $\Gamma_{\mathrm{i}}$, such that numerical values of $U_{\text {ii }}$ can be tabulated over a broad range of $\Gamma_{\mathrm{i}}$ 's [98, 99] and accurate formulae exist. The following formula interpolates between the low- $\Gamma$ Abe limit and the high- $\Gamma$ behavior [100]

$$
u_{\mathrm{ii}}^{\mathrm{eq}}(\mathbf{r})=k_{\mathrm{B}} T_{\mathrm{i}} \Gamma^{3 / 2}\left(\frac{A_{1}}{\sqrt{A_{2}+\Gamma}}+\frac{A_{3}}{1+\Gamma}\right),
$$

with $A_{1}=-0.9052, A_{2}=0.6322$ and $A_{3}=-\sqrt{3} / 2-A_{1} / \sqrt{A_{2}}$.

This expression is valid only for an OCP, i.e., a system of ions embedded in a neutralizing negative electron background. Therefore, ultracold plasma 
experiments provide an ideal testing ground for the validity of the widely used OCP model in real two-component systems. While the OCP model should yield a good description for $\Gamma_{\mathrm{e}} \rightarrow 0$, we would certainly expect deviations from Eq. (43) due to electron screening of the ion-ion interaction for low initial electron temperatures. For correlated but still weakly coupled electron components this effect is well described within the Debye-Hückel theory by an exponentially screened Yukawa-type interaction potential

$$
V_{\mathrm{ii}}=\frac{e^{2}}{4 \pi \varepsilon_{0}} \frac{e^{-r / \lambda_{\mathrm{D}}}}{r},
$$

where

$$
\lambda_{\mathrm{D}}=\sqrt{\varepsilon_{0} k_{B} T_{\mathrm{e}} /\left(e^{2} \rho_{\mathrm{e}}\right)}
$$

is the Debye screening length, characterizing the mean distance on which local charge imbalances are screened inside the plasma. As this screening effect introduces an additional length scale in the plasma the equilibrium correlation energy now depends on two parameters, $\Gamma_{\mathrm{i}}$ and $\kappa=a / \lambda_{\mathrm{D}}$,

$$
U_{\mathrm{ii}}^{(\mathrm{eq})}=k_{\mathrm{B}} T_{\mathrm{i}} \Gamma_{\mathrm{i}}\left(\tilde{U}+\frac{\kappa}{2}\right)
$$

The quantity $\tilde{U}\left(\Gamma_{\mathrm{i}}, \kappa\right)$ has been obtained from molecular dynamics simulations and tabulated over a wide range of $\Gamma_{\mathrm{i}}$ and $\kappa$ values [101,102].

The range of applicability of the Debye Hückel is not strictly defined. The theory is rigorously valid only for $a \ll \lambda_{\mathrm{D}}$. Ultracold plasma experiments, however, showed that Eq.(46) yields a surprisingly good description even for $a \approx \lambda_{\mathrm{D}}\left(\Gamma_{\mathrm{e}} \approx 1 / 3\right)$ - i.e. with only one electron per Debye sphere [12].

Eq. (41) and Eqs. (39) constitute a closed set of equations, describing the time evolution of a neutral plasma of electrons and ions. Compared to more involved approaches, such as, e.g., Molecular Dynamics methods, the macroscopic evolution equations are simple enough to allow for significant physical insight into effects of ion correlation on the plasma dynamics. While Eq. (39d) shows no direct influence on the electron temperature, the time evolution of the ion temperature is significantly modified by the interionic correlations. In addition to the adiabatic cooling, which is given by the first term on the right-hand side (rhs) of (39c) in complete analogy to the electronic component, the ionic temperature is increased (note that $U_{\mathrm{ii}}<0$ ) by the development of correlations (last term on the rhs of $(\underline{39 \mathrm{c}})$ ). This effect, which has been named "disorderinduced heating" or "correlation-induced heating," has been widely studied for homogeneous systems [80,37,41,40,103,104,73,30. Ultracold plasmas not only allow for experimental tests of present theories but also for studies of the ionic correlation dynamics in expanding systems with a steadily changing local equilibrium state, as described by the hydrodynamical evolution equations. 
The modified time evolution of the ionic temperature leads to a modified expansion dynamics of the plasma through the change in the ionic pressure, which contains a correlation part in addition to the thermal part. The development of ionic correlations also reduces of the total electrostatic interaction energy compared to a fully uncorrelated plasma. Together, these two effects result in the term $U_{\text {ii }} / 3$ on the rhs of (39b), which constitutes an effective negative acceleration slowing down the plasma expansion. This term can be regarded as a time dependent "external" force, giving rise to an additional effective potential in which the ions move. This potential, however, is not static, but changes during the expansion of the plasma, and leads (due to conservation of total energy) to an additional heating of the ions (second term on the rhs of (39c)).

Eqs. (39a)-(39c), (31d $)$ and (31e) form the final set of equations for the macroscopic parameters of the plasma state. They are quickly and easily solved numerically. Since the numerical effort is independent of the number of particles involved, the hydrodynamic approach provides a convenient method for simulations of large plasma clouds over long, i.e., millisecond, timescales. It can be used to quickly gain insight into the plasma dynamics by efficiently scanning a broad range of initial-state parameters, and it is able to simulate plasmas that are too large to be treated effectively with molecular dynamics methods. Moreover, and maybe even more importantly, the kinetic model provides physical insight by reducing the plasma dynamics to a few macroscopic parameters. However, the accuracy of these approximate macroscopic treatments, particularly when they describe dynamics influenced by ion correlation, must be assessed carefully. This can be done to some extent by comparison with experiment, but more rigorously by developing numerically accurate microscopic approaches relaxing most of the approximations invoked in the macroscopic approach.

\subsection{Microscopic Approaches}

Microscopic approaches are by definition more accurate. They are of great value for justifying macroscopic descriptions, and of course are the only alternatives for plasma conditions that preclude macroscopic descriptions. This refers to detailed information about the very early system evolution which is not accessible to hydrodynamics, and the effects of strong particle correlations, which are of particular interest in the context of ultracold plasmas. 


\subsubsection{Molecular Dynamics Simulations}

Molecular dynamics simulations of Coulomb-type interacting particles have been employed for investigating a variety of different problems in plasma physics and are a corner stone of astrophysical studies of galaxy and globular clusters [105]. Conceptually, the problem seems rather simple as it only involves the solution of a set of coupled differential equations describing the classical motion of charged particles. However, there are several technical challenges, which in the case of ultracold neutral plasmas arise from the open boundary of the expanding system, the attractive nature of the electron-ion interaction and the small mass ratio of the electrons and the ions.

A general difficulty for simulations of neutral plasmas arises from the bound state dynamics of atomic electrons, whose orbital timescale is many orders of magnitude smaller than the plasma evolution time. In particular for highly eccentric orbits conventional integrators, such as higher order Runge-Kutta methods, do not provide a stable solution, as they lead to a significant drift of the total energy. More appropriate propagation schemes such as symplectic integrators without energy drift and originally designed for the integration of gravitational systems, have clear advantages, but also several draw-backs and the method of choice depends on the particular problem to be studied. For simulating ultracold plasmas higher order symplectic integrators [106], predictor-corrector schemes [41] and timescaling methods combined with regularization procedures [107] can yield stable and reliable results.

Although a single electron and ion pair can be propagated very stably, manybody simulations have to fight additional complications. Electrons are bound to different ions with different binding energies and hence their orbital times can be very different. Moreover, these orbital times are generally much smaller than the timescale on which the heavier ions move. With a typical ion timescale on the order of microseconds, one has to cover timescales from femtoseconds to microseconds in order to describe the initial relaxation of the ionic component. Mazevet et al. [38] and Kuzmin and O'Neil [41] have used a small ion mass and scaled the obtained timescales to realistic experimental situations to obtain a quantitative estimate of the experimental results. Such a scaling, however, does not apply to all aspects of the plasma dynamics, since e.g., the timescales for electron-ion equilibration and ion-ion equilibration scale with a different power of the ion mass.

Several methods to numerically tackle very different timescales have been discussed in the literature (see, e.g., [108] and [105|109] for an overview and further references). The common idea of such approaches is to introduce individual timesteps for the different particles. The criteria determining the timestep and the procedures for the synchronization of the different particles can however be quite different and have to be adapted to the specific prob- 
lem under consideration. Kuzmin and O’Neil [4137] used a modified version of the method originally developed by Aarseth [110] to perform a full scale simulation of ultracold neutral plasmas with a realistic xenon ion mass and a total number of 8192 particles.

The number of particles $N$ is generally limited by the number of force evaluations that have to be carried out for every timestep. Since for every particle one has to sum up $N-1$ forces of the surrounding charges the numerical effort quickly increases as $N \cdot(N-1) \sim N^{2}$ with increasing number of particles. Simulations of large, extended plasmas typically consider a smaller part of the system and employ periodic boundary conditions to mimic the properties of the larger system [109. For ultracold plasmas such an approach is not very appropriate as it neglects an important part of the dynamics - the expansion of the plasma. The $N^{2}$-scaling of the numerical effort can, however, be reduced by so-called hierarchical tree-methods [111] or fast multipole methods [112, originally developed to simulate globular clusters. Such approaches still account for the exact forces of nearby charges but approximate the force of distant particles by the average force up to a certain multipole order of the corresponding charge distribution. This reduces the scaling of the numerical effort with the number of particles to $\sim N \ln N$ or even $\sim N$.

\subsubsection{Particle-In-Cell Approaches}

An alternative and conceptually different method to reduce the numerical effort connected with the summation of the inter-particle forces is to neglect correlations between the charges but retain their meanfield interaction due to the long-range part of the Coulomb potential. The so-called particle-in-cell simulations (PIC) [113] have become a standard method to treat various plasma physics problems, such as the collective dynamics of fusion devices [114,115] or of cold plasmas in gas discharges [116,117,118], i.e., weakly coupled plasmas. Clearly, for ultracold neutral plasmas this method seems questionable, as we are interested in the regime of strong coupling. However, the approach has proven to yield a reliable and efficient description of certain aspects of the plasma dynamics as long as the plasma expansion is mainly driven by the thermal pressure of the electrons [39].

The striking advantage of the PIC method is the possibility to treat very large systems, by replacing a collection of real particles by a single super-particle, with an increased charge and mass, retaining the charge-to-mass ratio of the physical particle. A single propagation timestep of such an ensemble of superparticles consists of the following sub-steps:

(1) At the beginning of each propagation timestep the positions of these super-particles are used to determine the charge density of the system, 
represented on an appropriately chosen grid. To reduce numerical noise due the finite particle number the charges are represented by a shape function. Most common shape functions are rectangles (particle in cell), tent functions (cloud in cell) or Gaussians centered around the actual particle position. Further problems arise for particle weighting in curved coordinates, as discussed in [119].

(2) Having obtained the charge density one evaluates the corresponding static electric field from the Poisson equation (Eq. 19). If the system exhibits additional spatial symmetries this is easily done by employing Gauss' theorem. In the general case the electric mean-field is most efficiently evaluated within an FFT procedure.

(3) The calculated electric field is subsequently transformed to the actual particle positions taking into account the chosen shape function, which finally allows advancement of all particle positions and velocities according to the classical equations of motion.

The positions and velocities of the test-particles have no physical meaning. The relevant quantities are the phase space distributions of the charges determined by the ensemble of test-particles. It is easy to see that the resulting dynamics provides a numerical solution of the Vlasov equation (18).

Hence, the PIC treatment as described so far does not account for any collisional processes, such as formation of Rydberg atoms and subsequent electronatom scattering. Such collisions can however be incorporated into the PICtreatment on the basis of the collision integrals introduced in Eqs. (27)(29). Within such so-called particle-in-cell-Monte-Carlo-collision simulations (PICMCC) [86] the collision terms in Eq. (18) are evaluated by random realizations of collision events, with a probability distribution determined by the respective collision integral.

For the collision integrals given in Eqs. (28) and (29) describing inelastic collisions and Rydberg atom formation, this stochastic evaluation procedure is particularly simple. First, one introduces an additional species by providing each ion with an additional label $n$, which characterizes its internal state. If the particle is an ion, then $n=0$, and $n>0$ for atoms, where $n$ denotes the principle quantum number of the atom. During each timestep of length $\Delta t$, an ion at position $\mathbf{r}$ is transformed into an atom with probability $P_{\text {tbr }}=\Delta t \sum_{n} K_{\text {tbr }}\left(n, \rho_{\mathrm{e}}\left(\mathbf{r}_{\mathrm{i}}\right), T_{\mathrm{e}}\right)$. In this case, the corresponding principal quantum number is sampled from the probability distribution $P_{\mathrm{tbr}}(n)=$ $\Delta t K_{\text {tbr }}\left(n, \rho_{\mathrm{e}}\left(\mathbf{r}_{\mathrm{i}}\right), T_{\mathrm{e}}\right) / P_{\mathrm{tbr}}$. At the same time the electron number is decreased by unity. Analogously, for bound states, an electron-atom collision occurs with the probability $P_{\text {ea }}=\Delta t\left(K_{\text {ion }}\left(n, \rho_{\mathrm{e}}\left(\mathbf{r}_{\mathrm{a}}\right), T_{\mathrm{e}}\right)+\sum_{p \neq n} K_{\mathrm{bb}}\left(n, p, \rho_{\mathrm{e}}\left(\mathbf{r}_{\mathrm{a}}\right), T_{\mathrm{e}}\right)\right)$. With a probability $P_{\text {ion }}=\Delta t K_{\text {ion }}\left(n, \rho_{\mathrm{e}}\left(\mathbf{r}_{\mathrm{a}}\right), T_{\mathrm{e}}\right) / P_{\text {ea }}$, this leads to ionization of the atom. Otherwise, the collision results in a bound-bound transition to a state with principal quantum number $p$ with probability $P_{\mathrm{bb}}(n, p)=$ 
$\Delta t K_{\mathrm{bb}}\left(n, p, \rho_{\mathrm{e}}\left(\mathbf{r}_{\mathrm{a}}\right), T_{\mathrm{e}}\right) / P_{\mathrm{ea}}$.

We finally note, that the tractable collision integrals are not limited to such a simple case, but can also be nonlinear in the distribution functions. In particular, elastic electron-ion and ion-ion collisions can be described in a similar way [33,94].

\subsubsection{Hybrid Methods}

So far we have discussed two complementary approaches which either provide the full microscopic information about the early plasma evolution or allow to follow the long-time dynamics on the cost of neglecting possible strong correlations between the plasma particles.

While the ionic component may indeed form a strongly correlated state, it turns out that the electrons remain weakly coupled during the entire plasma evolution (see Section 4.5 and 4.6). In [34,39] this different behavior of the two particle species has been used to develop a hybrid-molecular-dynamics approach. It treats the electrons and ions on different levels of approximations, combining the advantages of a mean field treatment (electron dynamics) and molecular dynamics simulations (ion motion). Collisions between electrons and the heavy particles (atoms and ions) are accounted for within the MonteCarlo procedure described in the previous subsection. By eliminating the short dynamical timescale of the bound electron motion this method significantly reduces the numerical workload, while simultaneously enabling a virtually exact numerical simulation of the ion dynamics fully incorporating ion-ion correlations.

The numerical effort may be further reduced within an adiabatic approximation for the electrons. As discussed in Section 3.1.1, the small electron-to-ion mass ratio permits an adiabatic approximation, where instantaneous (on the timescale of the ion dynamics) equilibration of the electrons is assumed. Keeping this approximation in a (hybrid) MD approach means that much larger timesteps may be chosen, since the electronic dynamics does not need to be resolved. In this way, an MD simulation of the ion motion over the whole timescale of the experiments becomes feasible. According to Eq. (20), the electronic distribution function is then given by a quasistatic distribution $f_{\mathrm{e}}^{(\mathrm{qs})}$ at all times.

The exact form of $f_{\mathrm{e}}^{\text {(qs) }}$, however, merits some discussion. The assumption of an ideal equilibrium, i.e. a Maxwell-Boltzmann distribution

$$
\rho_{\mathrm{e}} \propto \exp \left(\frac{e \varphi\left(\mathbf{r}_{\mathrm{e}}\right)}{k_{\mathrm{B}} T_{\mathrm{e}}}\right)
$$


leads to fundamental problems. Since the mean-field potential $\varphi$ has a finite depth, the corresponding Maxwell-Boltzmann distribution approaches a finite non-zero value even for $r \rightarrow \infty$ and hence is not normalizable. In [33], this problem was circumvented by introducing a spatially dependent electron temperature which is constant within a finite region around the plasma center, but decreases quadratically for large distances. An analogous problem was discussed long ago in an astrophysical context, in connection with the formation of star clusters, where the assumption of a Maxwellian velocity distribution implies an infinite mass of the star cluster in complete analogy to the present case. As discussed by Chandrasekhar [120], such a velocity distribution contains particles (stars) with arbitrarily high velocities which are not bound to the cluster potential. The rate with which these stars evaporate from the cluster has also been considered in [120], and later in [121]. If the evaporation is much slower than the relaxation of the stars remaining in the cluster, a quasistationary distribution forms. Comparison with numerical simulations shows that this distribution is well described by a truncated Maxwell-Boltzmann distribution

$$
f_{\mathrm{e}}\left(\mathbf{r}=\mathbf{0}, \mathbf{v}_{\mathrm{e}}\right) \propto \exp \left(-\frac{m_{\mathrm{e}} v_{\mathrm{e}}^{2}}{2 k_{\mathrm{B}} T_{\mathrm{e}}}\right)-\exp \left(-\frac{m_{\mathrm{e}} w_{\mathrm{e}}^{2}}{2 k_{\mathrm{B}} T_{\mathrm{e}}}\right)
$$

in the center of the cluster. The escape velocity $w_{\mathrm{e}}=\sqrt{2 e \delta \varphi / m_{\mathrm{e}}}$ is determined by the depth of the potential well, $\delta \varphi=\varphi(r \rightarrow \infty)-\varphi(\mathbf{0})$. Following [122], this may be generalized to arbitrary positions,

$$
f_{\mathrm{e}}\left(\mathbf{r}_{\mathrm{e}}, \mathbf{v}_{\mathrm{e}}\right) \propto \exp \left(\frac{e \varphi\left(\mathbf{r}_{\mathrm{e}}\right)}{k_{\mathrm{B}} T_{\mathrm{e}}}\right)\left[\exp \left(-\frac{m_{\mathrm{e}} v_{\mathrm{e}}^{2}}{2 k_{\mathrm{B}} T_{\mathrm{e}}}\right)-\exp \left(-\frac{m_{\mathrm{e}} w_{\mathrm{e}}^{2}}{2 k_{\mathrm{B}} T_{\mathrm{e}}}\right)\right]
$$

If the potential is a monotonic function of the distance from the cluster center, as in the case of gravitational forces, the space-dependent escape velocity is given by $w_{\mathrm{e}}=\sqrt{2 e\left[\varphi(\infty)-\varphi\left(r_{\mathrm{e}}\right)\right] / m_{\mathrm{e}}}$ for a spherical symmetric system, and one obtains the so-called Michie-King distribution [123]122]. In the present case of a neutral plasma, the potential may be non-monotonic due to the presence of charges with different sign. Hence, the escape velocity has to be defined more generally as

$$
\frac{m_{\mathrm{e}}}{2} w_{\mathrm{e}}^{2}=\max _{r \geq r_{\mathrm{e}}}\left[e \varphi\left(r_{\mathrm{e}}\right)-e \varphi(r)\right]
$$

With $W_{\mathrm{e}} \equiv m_{\mathrm{e}} w_{\mathrm{e}}^{2} /\left(2 k_{\mathrm{B}} T_{\mathrm{e}}\right)$, the electronic density is then given by

$$
\rho_{\mathrm{e}}\left(\mathbf{r}_{\mathrm{e}}\right)=\int_{0}^{w_{\mathrm{e}}} 4 \pi v_{\mathrm{e}}^{2} f_{\mathrm{e}} d v_{\mathrm{e}} \propto \exp \left(\frac{e \varphi\left(\mathbf{r}_{\mathrm{e}}\right)}{k_{\mathrm{B}} T_{\mathrm{e}}}\right) \int_{0}^{W_{\mathrm{e}}} e^{-x} x^{3 / 2} d x,
$$

which approaches a Maxwell-Boltzmann distribution in the center of the plasma for sufficiently low temperatures (i.e. $W_{\mathrm{e}} \gg 1$ ) but decays significantly faster towards the edge of the plasma. 
The Poisson equation, particle number conservation, energy conservation, and distribution Eq. (51) constitute a closed set of equations

$$
\begin{aligned}
\Delta \varphi & =4 \pi e\left(\rho_{\mathrm{e}}-\rho_{\mathrm{i}}\right) \\
\rho_{\mathrm{e}} & \propto \exp \left(\frac{e \varphi}{k_{\mathrm{B}} T_{\mathrm{e}}}\right) \int_{0}^{W_{\mathrm{e}}} e^{-x} x^{3 / 2} d x \\
N_{\mathrm{e}} & =\int \rho_{\mathrm{e}} d \mathbf{r}_{\mathrm{e}} \\
E_{\mathrm{ges}} & =\frac{m_{\mathrm{e}}}{2} \int v_{\mathrm{e}}^{2} f_{\mathrm{e}} d \mathbf{r}_{\mathrm{e}} d \mathbf{v}_{\mathrm{e}}-\int \rho_{\mathrm{e}} \varphi d \mathbf{r}_{\mathrm{e}}+E_{\mathrm{i}}+E_{\mathrm{a}}=\text { const. },
\end{aligned}
$$

that uniquely determines the electronic density, temperature, and mean-field potential $\varphi$ at all times. (In Eq. (52d), $E_{\mathrm{i}}$ is the sum of potential and kinetic energies of all ions, and $E_{\mathrm{a}}$ is the sum of the electronic binding energies and kinetic energies of all atoms.) Having calculated the electron density profile at time $t$, the knowledge of the corresponding electron mean field force is used to advance the ion positions and velocities under the influence of the electronic mean field and the full ion-ion interaction according to

$$
m_{\mathrm{i}} \ddot{\mathbf{r}}_{\mathrm{i}, j}=\mathbf{F}_{\mathrm{ei}}+\sum_{k \neq j} \mathbf{F}_{\mathrm{ii}}\left(\mathbf{r}_{\mathrm{i}, j}, \mathbf{r}_{\mathrm{i}, k}\right)
$$

where $\mathbf{r}_{\mathrm{i}, j}$ is the position of the $j$ th ion, $\mathbf{F}_{\mathrm{ei}}$ is the force due to the electronic mean-field,

and

$$
\mathbf{F}_{\mathrm{ei}}=-e \frac{\partial \varphi_{\mathrm{e}}}{\partial \mathbf{r}_{\mathrm{i}}} \quad ; \quad \Delta \varphi_{\mathrm{e}}=4 \pi e \rho_{\mathrm{e}}
$$

$$
\mathbf{F}_{\mathrm{ii}}\left(\mathbf{r}_{\mathrm{i}, j}, \mathbf{r}_{\mathrm{i}, k}\right)=\frac{\mathbf{r}_{\mathrm{i}, j}-\mathbf{r}_{\mathrm{i}, k}}{\left|\mathbf{r}_{\mathrm{i}, j}-\mathbf{r}_{\mathrm{i}, k}\right|^{3}} .
$$

In this propagation scheme it is still numerically quite costly to calculate the inter-ionic forces. However, as demonstrated in [30], with usage of hierarchical tree-methods for the direct propagation of the ions, this hybrid-MD approach allows the description of large systems, with $\sim 10^{6}$ ions over simulation times of several tens of microseconds.

\section{Physical Processes in Ultracold Neutral Plasmas}

The previous section presented the experimental and theoretical tools to generate, detect, and understand ultracold neutral plasmas. In this section we will use these tools to discuss physical processes. We proceed along the timeline of the dynamics (Fig. 4), i.e., we start in Section 4.1 with electron equilibration after photoionization. Then we will cover the establishment of local thermal equilibrium for the ions in Section 4.2. Before we come to the plasma expansion (Section 4.4) into the surrounding vacuum, we introduce collective 


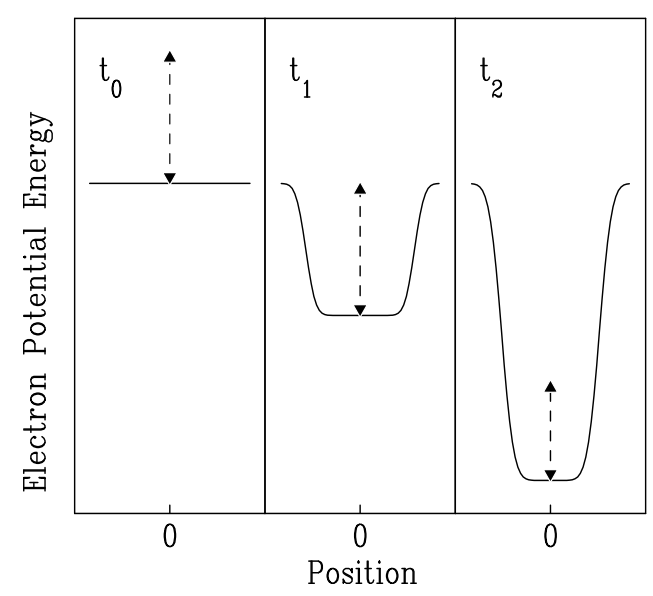

Fig. 9. Schematic of the potential energy seen by a test electron when enough atoms are photoionized to result in trapping of electrons. Photoionization occurs at $t_{0}=0$ and the sample is neutral everywhere. Because of the kinetic energy imparted by the laser, some electrons leave and a charge imbalance develops. At $t_{1} \approx 10 \mathrm{~ns}$ the resulting potential well equals the initial kinetic energy, trapping the remaining electrons. As electrons in the well thermalize, evaporation occurs. The well depth increases and the electrons cool slightly. By $t_{2} \approx 1 \mu$ s evaporation essentially stops. The dashed line indicates the average kinetic energy of the electrons. Reused with permission from [2]. Copyright 1999, American Physical Society.

excitations of the plasma electrons, which may be used to probe the plasma expansion dynamics (Section 4.3).

Two more subsections are devoted to important topics. First, a detailed analysis of the initial heating of the electrons, which affects the expansion dynamics is given in Section 4.5. Secondly, Section 4.6 will discuss in some detail the evolution of the electronic and ionic Coulomb coupling parameters, which are important since they indicate the degree of correlations that may be realized in a plasma.

\subsection{Initial Electron Equilibration}

\subsubsection{Trapping of Electrons}

The first experimental study of ultracold neutral plasmas [2] explored the creation of the plasma and trapping of the electrons by the ionic background charge. (See Fig. 9.) Immediately after photoionization, the charge distribution is neutral everywhere. Due to the kinetic energy of the electrons, the electron cloud expands on the timescale of the inverse electron plasma frequency $\tau_{\mathrm{e}}=\omega_{\mathrm{p}, \mathrm{e}}^{-1}=\sqrt{m_{\mathrm{e}} \varepsilon_{0} / \rho_{\mathrm{e}} e^{2}} \sim 1 \mathrm{~ns}$, where $\rho_{\mathrm{e}}$ is the electron density, and $\omega_{\mathrm{p}, \mathrm{e}}$ is the electron plasma oscillation frequency. On this timescale the ions are essentially immobile. The resulting local charge imbalance creates a Coulomb 


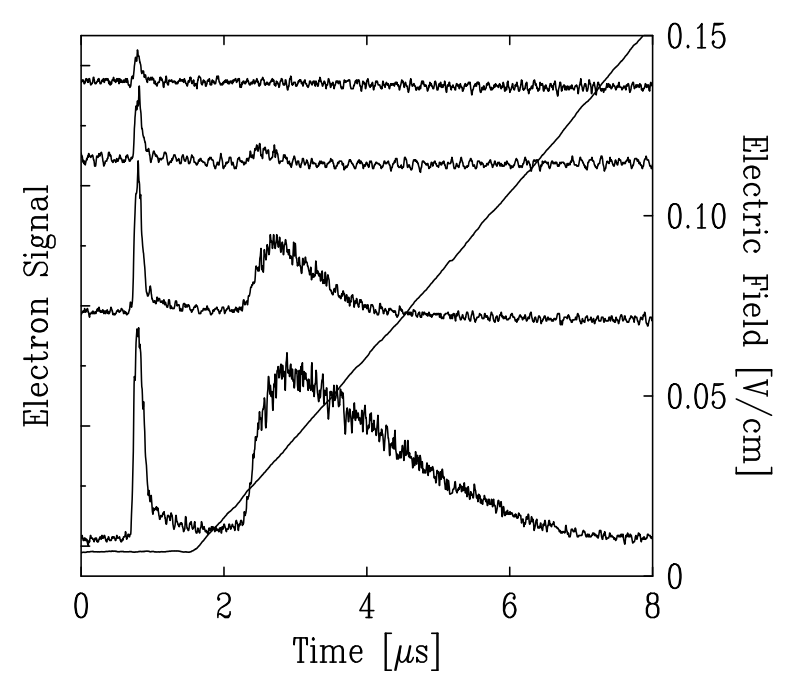

Fig. 10. Electron signals recorded for four different pulse energies of the photoionizing laser, i.e., different densities of charged particles $\left(10^{5}-10^{7} \mathrm{~cm}^{-3}\right)$. The uppermost curve corresponds to the lowest energy. The photoionization occurs at $t=0$. The initial kinetic energy of the electrons is $\Delta E / k_{B}=0.6 \mathrm{~K}$. The data shown is an average over 20 cycles of the experiment. Also shown is the magnitude of the applied electric field. Reused with permission from [2]. Copyright 1999, American Physical Society.

potential energy well that traps all but a small fraction $(<5 \%)$ of the electrons. Smaller clouds, more ions and electrons, and lower initial electron kinetic energy all lead to a larger fraction of electrons trapped by the ions. Simple PICC simulations [2] show that electrons escape mostly from the edges of the spatial distribution.

This description implies that there is a threshold for trapping electrons. If the well never becomes deeper than the initial kinetic energy, all the electrons escape. This is demonstrated in Fig. 10, and the diagnostic used to obtain this data was charged particle detection of electrons after they have left the plasma, as shown in Fig. 5. In each cycle of the experiment, the atoms are first laser cooled and an electric field of approximately $5 \mathrm{mV} / \mathrm{cm}$ is applied. Subsequently, the atoms are photoionized, and after about $500 \mathrm{~ns}$ of time of flight a pulse of electrons arrives at the detector (see the first peak in the curves of Fig. 10). A few microseconds later, the electric field is linearly increased. If electrons have been trapped by the ionic background potential, they will be liberated now to produce the second peak in the curves of Fig. 10, If the laser intensity is so low that the well never becomes deeper than the initial kinetic energy, no electrons are trapped and the second peak is missing (top trace). In addition, when electrons are trapped, the first peak develops a tail. Trapped electrons thermalize within $10-100 \mathrm{~ns}$ [60, and as charges are promoted to energies above the trap depth, they leave the well. On the time scale of this experiment, the ions are essentially stationary. 


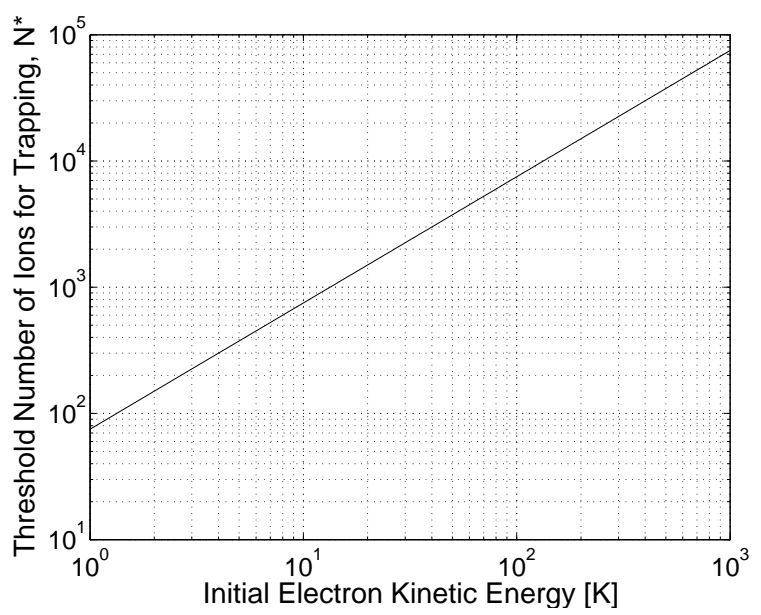

Fig. 11. $N^{*}$, threshold number of ions required for trapping electrons for a Gaussian plasma with $\sigma=1 \mathrm{~mm}$.

One can quantify the threshold for the trapping effect. The last electron to leave the plasma must climb out of the potential formed by the ions, with no neutralizing electrons. At threshold, the depth of this well equals $E_{\mathrm{e}}$. The density profile of the ions follows that of the neutral atoms Eq. (2), where the maximum density in the center is given by $\rho_{\mathrm{i}}=N_{\mathrm{i}} /\left(2 \pi \sigma^{2}\right)^{3 / 2}$ with the total number of ions, $N_{\mathrm{i}}$. The electrostatic potential produced by this charge distribution is

$$
U\left(r, N_{\mathrm{i}}\right)=-\int \frac{e^{2}}{4 \pi \varepsilon_{0}\left|\mathbf{r}-\mathbf{r}^{\prime}\right|} \rho_{\mathrm{i}}\left(r^{\prime}\right) d \mathbf{r}^{\prime}=-N_{\mathrm{i}} \frac{e^{2}}{4 \pi \varepsilon_{0} r} \operatorname{erf}\left(\frac{r}{\sqrt{2 \sigma}}\right)
$$

where $\operatorname{erf}(x)$ is the error function. The depth of the potential well is given by its value at the center $r=0$,

$$
U\left(0, N_{\mathrm{i}}\right)=-\frac{N_{\mathrm{i}} e^{2}}{4 \pi \varepsilon_{0} \sigma} \sqrt{\frac{2}{\pi}} .
$$

When $-U\left(0, N_{\mathrm{i}}\right)$ equals the electron kinetic energy, $E_{\mathrm{e}}$, then the last electron to leave the well is trapped. In other words, the minimum number of ions required to trap any electrons, defined as $N^{*}$, is found from $E_{\mathrm{e}}=-U\left(0, N^{*}\right)$. Figure 11 shows typical values of $N^{*}$.

The threshold condition $N_{\mathrm{i}}=N^{*}$ is mathematically equivalent to $\lambda_{D}=\sigma$, where $\lambda_{D}$ is the Debye screening length as defined in Eq. (45) with $T_{\mathrm{e}}=$ $\frac{2}{3} E_{\mathrm{e}} / k_{\mathrm{B}}$. Note, that the relation $\sigma>\lambda_{\mathrm{D}}$ is the standard condition for a system to be considered a plasma [124], namely that the system size $(\sigma)$ has to be larger than the characteristic length scale $\left(\lambda_{\mathrm{D}}\right)$ on which local charge imbalances are screened. In the present context, we can also interpret $\lambda_{D}$ as the displacement of electrons from their equilibrium positions when their energy in the local internal electric field in the plasma equals their kinetic energy [125]. 


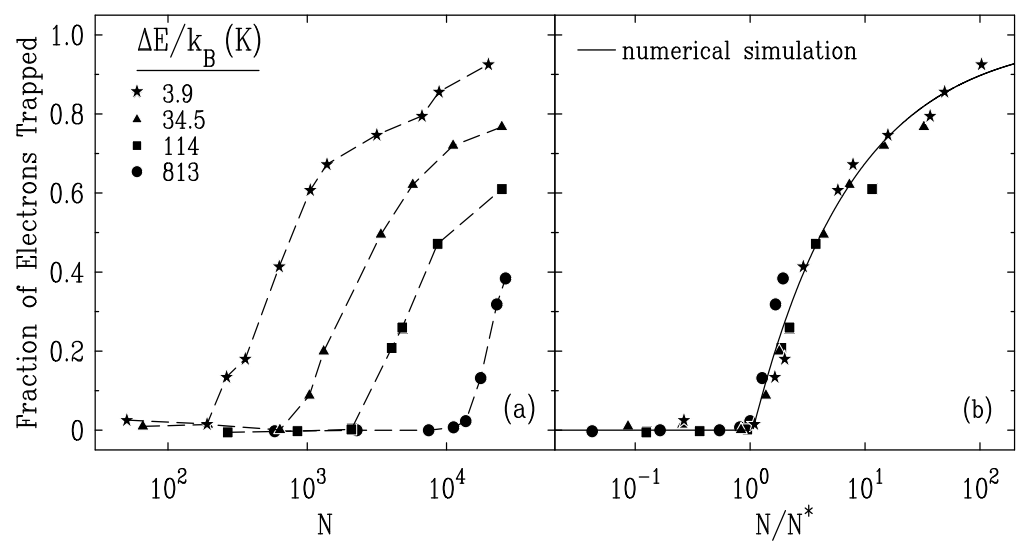

Fig. 12. (left) The fraction of electrons trapped is plotted versus the number of photoions created. Each curve corresponds to a different (green) laser frequency. The corresponding initial energies of the electrons are displayed in the legend. (right) Same as (left) but the number of ions is scaled by $N^{*}$, see text. There is a scale uncertainty of about $10 \%$ in determining the fraction of electrons trapped. Reused with permission from [2]. Copyright 1999, American Physical Society.

If $\lambda_{D}>\sigma$, the electrons are free to escape to infinity. If $\lambda_{D}<\sigma$, electrons are trapped by the ion cloud. In the center of a typical ultracold neutral plasma, $\lambda_{D}=1-10 \mu \mathrm{m}$. Typical plasma sizes are on the order of some hundred $\mu \mathrm{m}$, such that the requirement for the existence of a plasma state is well fulfilled under experimental conditions.

Fig. 12 [2] illustrates the trapping effect and the role of $N^{*}$. We see the number of ions required to begin trapping electrons grows with increasing $E_{\mathrm{e}}$ (left panel), i.e., the threshold $N^{*}$ increases. When the number of ions is scaled by $N^{*}$, the fraction of electrons trapped follows a universal curve, clearly exhibiting the threshold $N_{\mathrm{i}} / N^{*}=1$ (right panel). For $N_{\mathrm{i}}<N^{*}$ no electrons are trapped. This figure also shows that for $N / N^{*} \gg 1$, the non-neutrality is small with the fraction of trapped electrons almost reaching unity.

The fraction of trapped electrons is surprisingly well described by the simple formula [47,1]

$$
\frac{N_{\mathrm{e}}}{N_{\mathrm{i}}}=\frac{\sqrt{N_{\mathrm{i}} / N^{\star}}-1}{\sqrt{N_{\mathrm{i}} / N^{\star}}} .
$$

An analytic derivation of this dependence has not yet been found.

A theoretical discussion assuming a quasi-neutral plasma gives a better understanding of the degree and nature of non-neutrality in the plasma. First note that the quasi-neutrality condition does not imply an exact equality of the electron and ion densities and hence a vanishing space charge potential according to the Poisson equation (19). Instead, the Poisson equation is replaced by the electron Vlasov equation which in adiabatic approximation yields for 

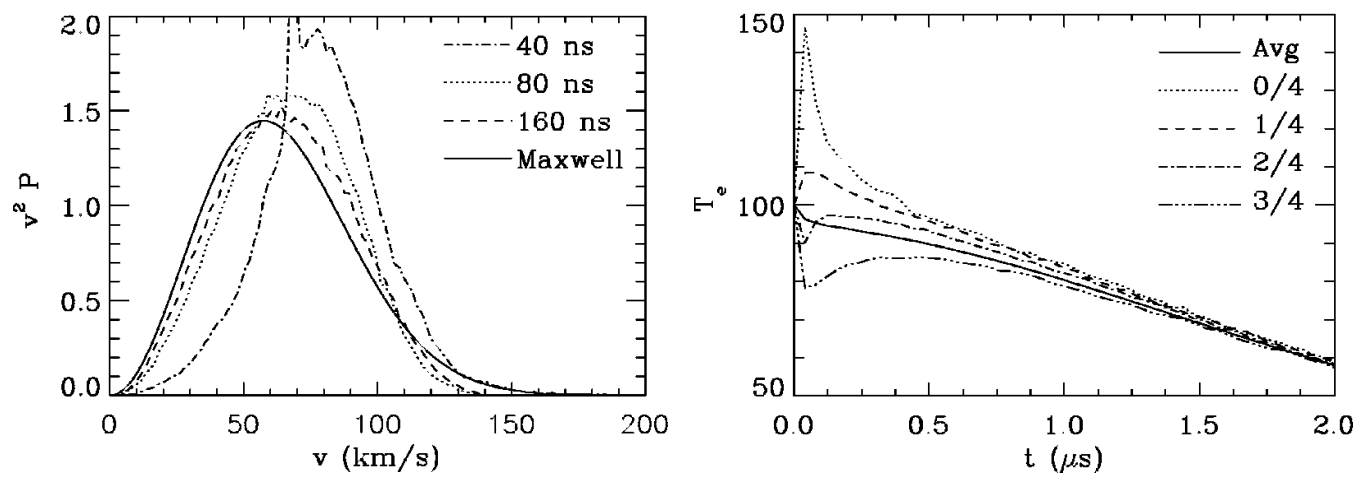

Fig. 13. (a) Electron velocity distribution at $t=40 \mathrm{~ns}$ (dash-dotted line), $t=80 \mathrm{~ns}$ (dotted line) and $t=160 \mathrm{~ns}$ (dashed line) compared to a Maxwell distribution (solid line). The distribution has been sampled from a shell $1 / 4$ out from the center of the plasma. (b) Time evolution of the electron temperature at the plasma center (dotted line), 1/4 out from the center (dashed line), 2/4 out from the center (dash-dotted line) and $3 / 4$ out from the center (dash-dot-dot-dot line). The solid line shows the average temperature of the innermost $80 \%$ of the electrons. The initial state parameters are $\bar{\rho}_{\mathrm{e}}=10^{9} \mathrm{~cm}^{-3}, T_{\mathrm{e}}=100 \mathrm{~K}$ and $\sigma=196 \mu \mathrm{m}$. Reused with permission from 33. Copyright 2003, American Institute of Physics.

the space charge potential (Eq. (21))

$$
e \varphi(r)=-k_{\mathrm{B}} T_{\mathrm{e}} \ln \frac{\rho_{\mathrm{i}}(r)}{\rho_{\mathrm{i}}(r=0)}=\frac{1}{2} k_{\mathrm{B}} T_{\mathrm{e}} \frac{r^{2}}{\sigma^{2}} .
$$

Now we can use this expression for a backward calculation of the charge separation from the Possion equation, which gives a spatially constant excess charge of

$$
\rho_{\mathrm{i}}-\rho_{\mathrm{e}}=3 \frac{k_{\mathrm{B}} T_{\mathrm{e}} \varepsilon_{0}}{e^{2} \sigma^{2}}
$$

Introducing the local Debye length, which is a function of density and thus radius, we find from Eq. (60), that the relative charge difference, i.e., the local degree of non-neutrality

$$
\frac{\rho_{\mathrm{i}}-\rho_{\mathrm{e}}}{\rho_{\mathrm{e}}}=\frac{\lambda_{\mathrm{D}}^{2}(r)}{\sigma^{2}}
$$

is solely determined by the ratio of the Debye length and the system size - just like the global non-neutrality (Eq. (58)). The fractional non-neutrality is small as long as $\lambda_{D}<\sigma$, typically less than $1 \%$ in the center of the plasma, where the density is high. It becomes large near the edge, however, violating the quasi-neutrality assumption, which may lead to deviations from the Gaussian density profile (see Section 4.4.4 for a more detailed discussion). For the major parts of the plasma volume $\lambda_{\mathrm{D}}^{2}(r)<\sigma^{2}$, justifying the statement that the plasma is essentially in a quasineutral state.

Non-neutrality is also related to a spatially dependent electron temperature. When using the definition of the Debye length $\lambda_{\mathrm{D}}$ in the above argumentat 
we have implicitly assumed the existence of an electronic temperature. While this is certainly a good approximation, a global thermal electron velocity distribution is just developing during the initial phase of the plasma relaxation. PICMCC simulations (Section [3.2.2), which yield very good agreement with the measured evaporating electron flux [33], show that the velocity distribution in the central plasma region quickly reaches its Maxwellian form within a few hundred $100 \mathrm{~ns}$ (see Fig. 13a). As shown in Fig. 13b it takes considerably longer for a global equilibrium to develop. The center of the plasma is seen to heat up, while the outer region cools down. The total electron kinetic energy is, however, almost unaffected by these local temperature imbalances. In reality, the electron gas heats up due to the formation of Rydberg atoms [66, 42,33,39] and an initial build-up of spatial electron correlations. The latter process takes place simultaneously with the initial electron evaporation, but is not observable in Fig. 13, due to the inability of the PIC treatment to describe the correlated dynamics of the electrons. A more detailed discussion of the various electron heating mechanisms will be given in Section 4.5.

\subsubsection{Spontaneous Ionization of a Dense Rydberg Gas}

Compared to photoionization of a trapped cloud of ground state atoms, the dynamics of plasma formation is more complicated when it is created by spontaneous ionization in a dense cloud of ultracold Rydberg atoms. Reference [56] showed that some Rydberg atoms are initially ionized by black-body radiation, collisions with hot background atoms, or Penning collisions with other Rydberg atoms. Attractive dipole-dipole and van der Waals interactions that pull Rydberg atoms together and enhance the Penning ionization rate are particularly important for high principal quantum number $(n>50)$ [126]. Resulting electrons escape the cloud in the first few microseconds until a few thousand ions build up to form a Coulomb well that traps subsequently produced electrons. This is reminiscent of the model presented in Fig. 9. At this point, the electrons seed an ionization avalanche. As much as $2 / 3$ of the initial Rydberg population is ionized, and the remaining fraction is collisionally de-excited to lower Rydberg levels, satisfying energy conservation [55]. During the avalanche, $l$-changing electron-Rydberg collisions, $n$-changing electronRydberg collisions, electron-Rydberg ionizing collisions, and Penning ionizing Rydberg-Rydberg collisions all play a role [55127]. By exciting Rydberg atoms with a relatively low density in a pre-existing ultracold plasma, it was shown in [68] that very tightly bound Rydberg atoms $(n \approx 20)$ could be ionized by electron-Rydberg collisions in these systems. The energetics of electrons in plasmas created in this way, such as the equilibrium temperature after the first few tens of nanoseconds, is a question that has not been well-explored. Theory [33] predicts no drastic change as the excitation laser crosses the ionization threshold and the experiment changes from direct photoionization to

initial excitation of Rydberg atoms. Recent experimental work supports this 


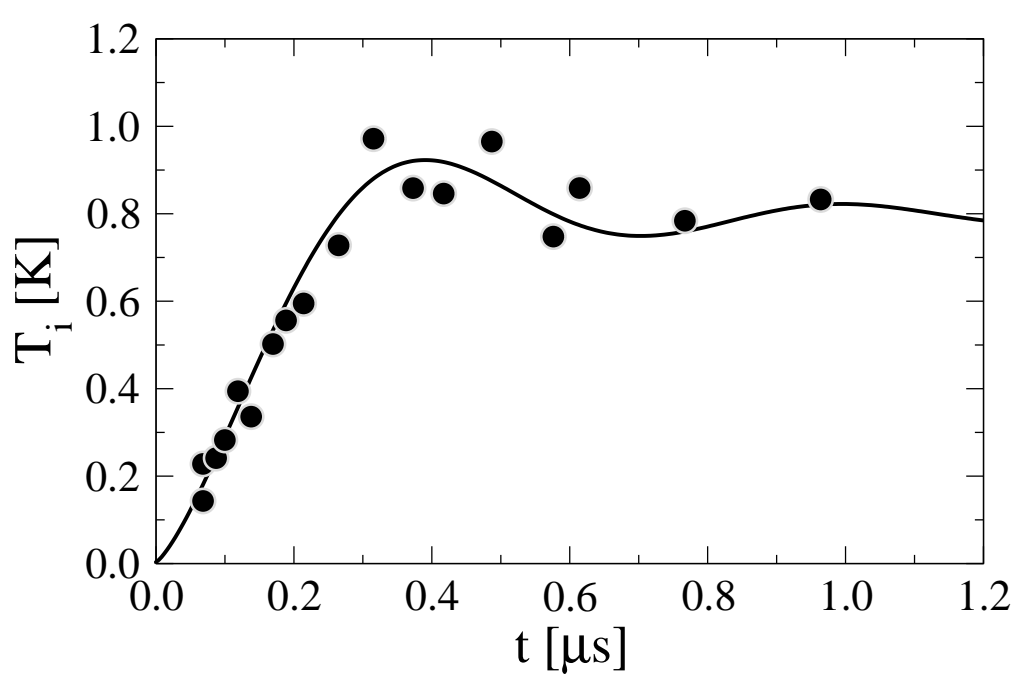

Fig. 14. Time evolution of the effective ion temperature for a Sr plasma with initial peak density $\rho_{0}(0)=2 \cdot 10^{9} \mathrm{~cm}^{-3}$ and electron temperature $T_{\mathrm{e}}(0)=38 \mathrm{~K}$. The results of a hybrid-MD calculation (solid line) are compared to experimental data extracted from an absorption imaging measurement (dots). Reused with permission from [30]. Copyright 2005, American Physical Society.

prediction [70].

\subsection{Initial Ion Equilibration}

The establishment of LTE for the ions occurs on a longer timescale than it does for the electrons, and it is conveniently studied experimentally. In addition, several inelastic processes (most notably three-body recombination) that heat the electrons do not affect the ions. In this unusual regime, interesting phenomena are expected to be observable.

Theoretically, the ion dynamics can be studied within the Hybrid Molecular Dynamics approach outlined in Section 3.2.3. In this approach, the ions are propagated individually, so that full information about their positions, velocities, as well as spatial correlations is available. Experimentally, on the other hand, the optical techniques described in Section 2.2 .2 provide an excellent tool for following the ion dynamics.

\subsubsection{Disorder-induced heating}

Figure 14 shows that the experimental evolution of the ion temperature of an ultracold plasma and the theoretical curve obtained from a hybrid-MD simulation are in good quantitative agreement over a time relevant for ion thermalization [30]. The most striking observation from Fig. 14] is the rapid heating of the 
plasma ions by several orders of magnitude, which is the so-called "disorderinduced heating" or "correlation heating." It originates from the fact that the system is created in an undercorrelated state far from thermodynamic equilibrium. Immediately after photoionization, the ions in an ultracold neutral plasma have very little kinetic energy. But they are spatially uncorrelated, and there is significant excess potential energy in the system compared to the equilibrium state in which spatial correlations keep ions from being close to each other [128,11]. During early evolution of the plasma, correlations develop and potential energy is converted into (thermal) kinetic energy [40,103,104] leading to equilibration. However, during the disorder-induced heating phase, the plasma is not in LTE, and the notion of an ion "temperature" should be interpreted as a measure of the average ion kinetic energy.

Within the framework of the hydrodynamical model of Section 3.1.3, the above interpretation of disorder-induced heating is immediately apparent in Eq. (39c): As long as expansion of the plasma is still negligible $(\gamma=0)$, the change in ion temperature is directly determined by the build-up of ion-ion correlations (last term on the right-hand side of (39c)). This process has been discussed in theoretical papers in many contexts. Early interest was generated by non-equilibrium plasmas created by fast-pulse laser irradiation of solid targets [129,130,131,80,81,132]. Experimental results were lacking, however, because of the fast timescales involved and limited possibilities for diagnostics. Ultracold neutral plasmas - much better suited for a detailed study of the process - reinvigorated interest in the mechanism of disorder-induced heating [37, 38, 40, 103, 104, 133].

Qualitatively, one expects the timescale for the ion heating to be given by the inverse of the ionic plasma frequency, $\omega_{\mathrm{p}, \mathrm{i}}^{-1}$, which is the typical timescale on which spatial correlations develop in a plasma. Physically, $\omega_{\mathrm{p}, \mathrm{i}}^{-1}$ is the time for an ion to move one interparticle spacing when accelerated by a typical Coulomb force of $e^{2} / 4 \pi \varepsilon_{0} a^{2}$, where $a=\left(4 \pi \rho_{\mathrm{i}} / 3\right)^{-1 / 3}$ is the Wigner-Seitz radius. For the typical plasma of Fig. 14, $\omega_{\mathrm{p}, \mathrm{i}}^{-1} \approx 270 \mathrm{~ns}$ in good agreement with the rise-time of the temperature observed in the figure. This timescale of $\sim 10^{2} \mathrm{~ns}$ is several orders of magnitude larger than in laser-generated highdensity plasmas as mentioned above, and it is easily resolved experimentally. One would guess the ion temperature after equilibration would be on the order of the Coulomb interaction energy between neighboring ions,

$$
k_{B} T_{C} \approx \frac{e^{2}}{4 \pi \varepsilon_{0}} \frac{1}{a} \equiv U_{a}
$$

which is a few kelvin. A quantitative analysis [40], assuming complete initial disorder and incorporating the screening effects of the electrons, predicts from a simple energy conservation argument an equilibrium ion temperature of 

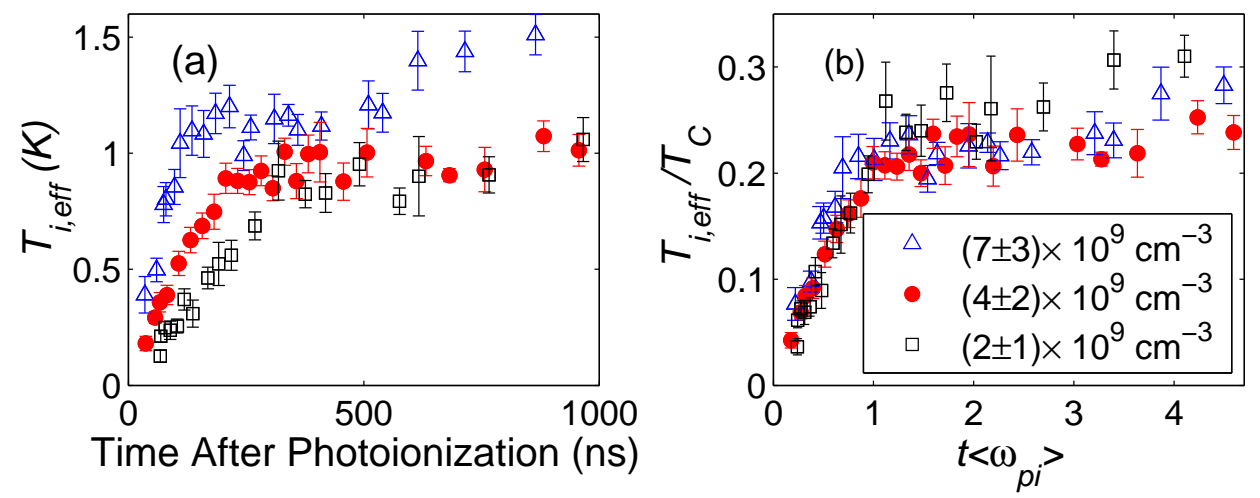

Fig. 15. The effective ion temperature, $T_{\mathrm{i}, \text { eff }}(\mathrm{Eq}$. (12)), versus time after photoionization for initial electron temperature of $T_{e}=2 E_{e} / 3 k_{B}=38 \pm 6 \mathrm{~K}$ and various plasma densities. (a) The data is plotted on absolute temperature and time scales. (b) The time is scaled by the inverse of the average plasma period, and $T_{\mathrm{i} \text {,eff }}$ is scaled by $T_{\mathrm{C}}$ (Eq. 62). Reused with permission from [28]. Copyright 2004, American Physical Society.

$$
T_{\mathrm{i}}=\frac{2}{3 k_{B}} U_{a}\left|\frac{\tilde{U}}{U_{a}}+\frac{\kappa}{2}\right| .
$$

Here, $\kappa=a / \lambda_{D}$ is the Debye screening constant, and $\tilde{U}$ is the excess potential energy per ion. $\tilde{U}$ has been studied with molecular dynamics simulations [134] for a homogeneous system of particles interacting through a Yukawa potential, $\phi(r)=U_{a}(a / r) \exp \left(-r / \lambda_{D}\right)$, which describes ions in the background of weakly coupled electrons [135]. To obtain $T_{\mathrm{i}}$ for given $\rho_{\mathrm{i}}$ and $T_{\mathrm{e}}$, one needs to selfconsistently solve Eq. (63) with an analytic expression for $\tilde{U}[102$ since $\tilde{U}$ depends itself on $T_{\mathrm{i}}$.

The arguments given above imply that both the timescale and the energy scale of the disorder-induced heating depend on the plasma density only (apart from a small dependence of the final temperature on the screening $\kappa$, which will be discussed below). Fig. 15 confirms this expectation. If the measured effective ion temperature $T_{\mathrm{i} \text {,eff }}$ is scaled with $T_{C}$ (Eq. (62)) and time with the inverse plasma frequency $\omega_{\mathrm{p}, \mathrm{i}}$, the measured curves for different densities coincide quite well (Fig. 15(b)). Small differences in the curves are due to electron screening of the ion-ion interaction. The effect of electron is demonstrated in Fig. 16, where $T_{\mathrm{i} \text {,eff }}$ obtained from absorption spectra (Eq. (12)) is shown for different initial electron energies but the same ion density distribution: Smaller initial electron energy (temperature) implies more screening and a lower equilibrium ion temperature. The latter is directly related to the initial excess potential energy (Eq. (63) ), which is lowered if screening through electrons is increased. It was found that the measured ion temperature just after the plasma reaches LTE agrees well with the theoretical value $T_{\mathrm{i} \text {,ave }}$ according to Eq. (63) and averaged over the ion distribution [28]. 


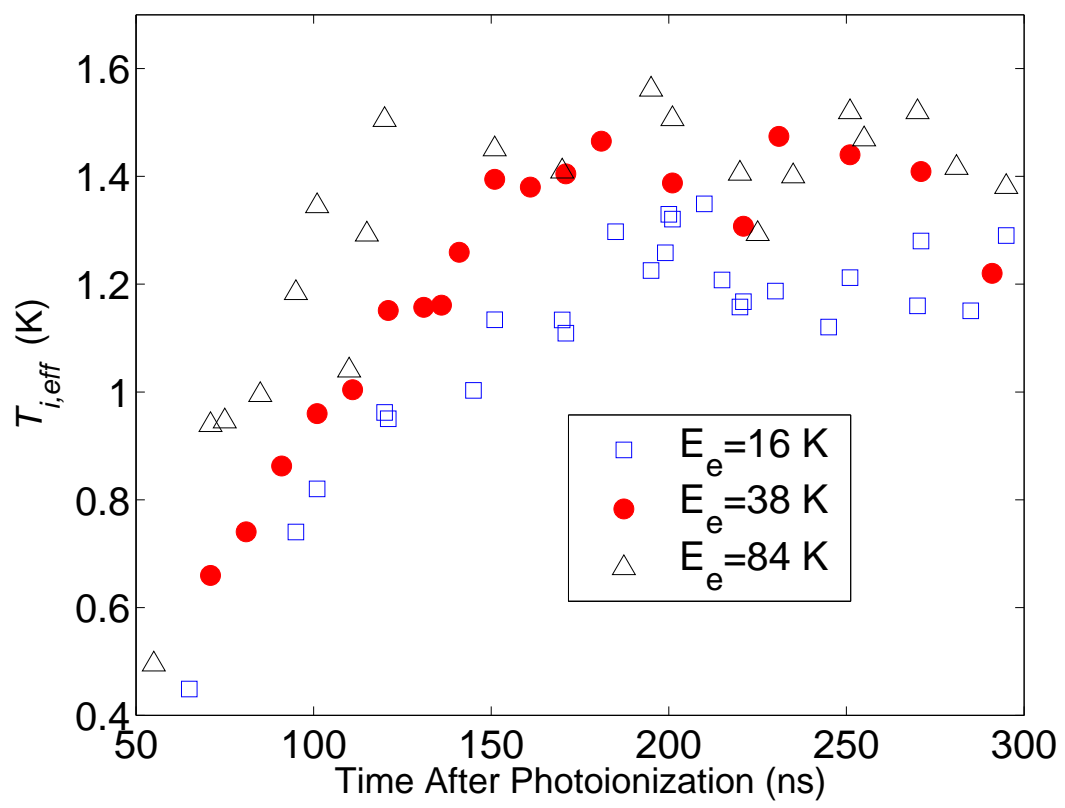

Fig. 16. Effective ion temperature versus time after photoionization for various initial electron kinetic energies. The peak ion density for all data is $\rho_{0 \mathrm{i}}=(1.4 \pm 0.5) \times 10^{10} \mathrm{~cm}^{-3}$. Reused with permission from [28]. Copyright 2004, American Physical Society.

As emphasized in [133], it is not surprising that that Eq. (63) accurately predicts the ion temperature for equilibrating ultracold neutral plasmas, because it basically expresses energy conservation. However, it is worth pointing out that for data in Ref. [28] with the lowest fit $T_{\mathrm{e}}$ and highest $\rho_{0 \mathrm{i}}$, the peak value of $\kappa$ in the plasma is 0.7 . This corresponds to three electrons per Debye sphere $\left(\kappa^{-3}=\rho_{\mathrm{e}} 4 \pi \lambda_{D}^{3} / 3\right)$. One might not necessarily expect Eq. (63) to be accurate in this regime because it assumes a Yukawa potential for ion-ion interactions, which is based on Debye screening and is normally derived for $\kappa^{-3} \gg 1$.

\subsubsection{Kinetic energy oscillations}

A close inspection of Figs. 15 and 16 reveals that at the end of the disorderinduced heating phase, the ion temperature overshoots its equilibrium value before settling to it. This phenomenon is even more evident in Fig. 17, where $T_{\mathrm{i}, \text { eff }}$ is shown for an inner and outer region of the plasma image $\left(\rho=\sqrt{x^{2}+y^{2}}<\right.$ $0.9 \sigma$ and $\rho>1.48 \sigma$, respectively). Each selected annular region contains $1 / 3$ of the ions and probes a region with significantly less variation in density than in the entire plasma. The region with lower density has lower ion temperature, as expected from Eq. (63), but the oscillation is the most striking observation. The oscillation was also observed in a calcium plasma with fluorescence measurement [13] (Fig. 18). The contrast of the oscillations is strong in this case because the small volume of the plasma excited by the fluorescence excitation laser significantly reduced the variation of the sampled density. Numerical 


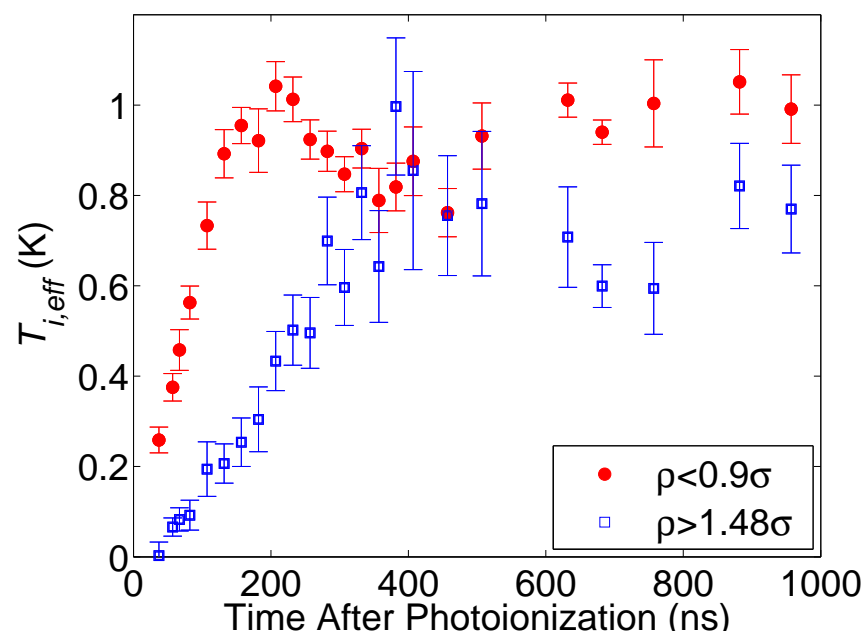

Fig. 17. Effective ion temperature obtained from different selected regions of a plasma cloud with $\rho_{0 \mathrm{i}}=(4 \pm 2) \times 10^{9} \mathrm{~cm}^{-3}$ and initial $T_{\mathrm{e}}=2 E_{\mathrm{e}} / 3 k_{B}=38 \pm 6 \mathrm{~K}$. Oscillations are clearly visible at the end of the disorder-induced heating process. Reused with permission from [28]. Copyright 2004, American Physical Society.

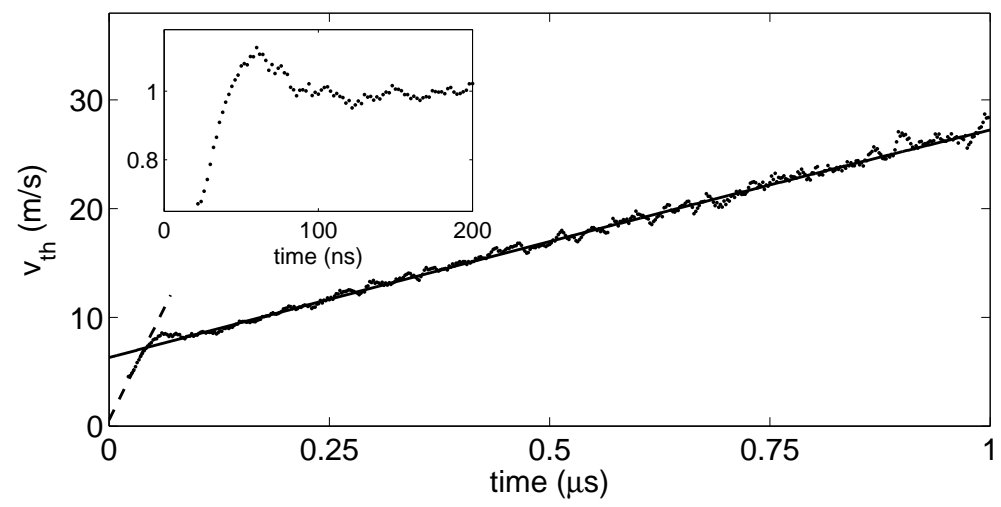

Fig. 18. The mean $z$-component of the ion velocity in a calcium plasma as a function of time after photoionization in a region defined by the excitation laser beam. The velocity was extracted using Eq. (17), and the absolute calibration was obtained by assuming the ions had an initial velocity distribution equal to that of the laser-cooled atoms. The rapid increase of the velocity at early times is due to disorder-induced heating. The subsequent slower increase reflects the expansion of the plasma, which will be discussed in Section 4.4. The solid line is a fit of the velocity to a model for the plasma expansion plus an offset representing the random thermal ion velocity. The inset shows the ion velocity divided by the fit, which highlights the oscillation of the kinetic energy. Reused with permission from [13. Copyright 2005, American Physical Society.

simulations clearly show the oscillations and also provide their temperature and spatial dependence (Fig. 19).

These oscillations display universal relaxation dynamics of a strongly coupled Coulomb system. More precisely, they reflect the fact that the two-particle 


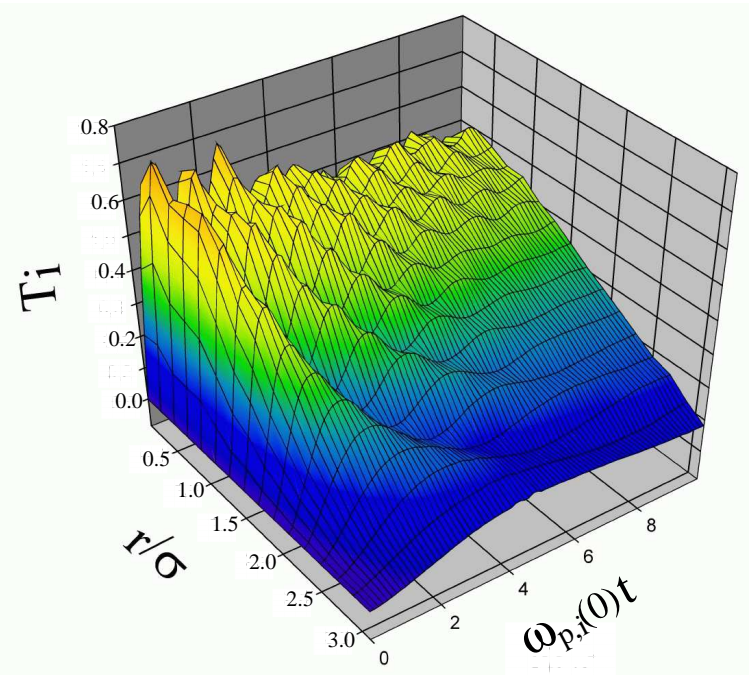

Fig. 19. Spatio-temporal evolution of the ion temperature, obtained from HMD-simulations for 40000 ions and electrons with an initial Coulomb coupling parameter of $\Gamma_{\mathrm{e}}(0)=0.07$. Reused with permission from [29]. Copyright 2005, Institute of Physics.

distribution function (i.e. spatial correlations) and the one-particle distribution function (i.e. temperature) relax on the same timescale $\left(1 / \omega_{\mathrm{p}, \mathrm{i}}\right)$ due to the strong long-range interactions. Normally, correlations relax much more quickly, and the separation of these time scales is known as the Bogoliubov assumption [136]. Studies with ultracold neutral plasmas represent the first experimental observation of kinetic energy oscillations, but it has been the subject of intense theoretical study through analytic calculations [130] and simulations [129, 131,80,81,132,133,30] of equilibrating strongly coupled plasmas. Calculations [129]137] also show oscillations in the velocity autocorrelation function in equilibrium systems.

Intuitively, one may relate this phenomenon to the motion of each ion in its local potential energy well. The discussion of disorder-induced heating in the previous section qualitatively ascribed the increase in ion kinetic energy to each ion moving towards the minimum of its well. The time scale for this motion is $\omega_{\mathrm{p}, \mathrm{i}}^{-1}$. However, the ion will not suddenly stop at the bottom of its potential; it will overshoot and climb up the hill again. It is reasonable to expect harmonic motion to persist for some time, as kinetic and potential energy are interchanged. This suggests a kinetic energy oscillation at $2 \omega_{\mathrm{p}, \mathrm{i}}$, which is in line with the observations. Also consistent with this description, the oscillation period observed experimentally in Fig. 17 is longer in the outer region where the average density is lower and hence the plasma frequency is smaller. This also explains why averaging over the entire cloud such as in Fig. 14 obscures the oscillation: the motion dephases because of the variation in $\omega_{\mathrm{p}, \mathrm{i}}$. In a disordered system corresponding to the ultracold plasma scenario, there is probably no collective or long-range coherence to the motion, so in 
spite of the fact that $\omega_{\mathrm{p}, \mathrm{i}}$ sets the timescale of the oscillation, one must be cautious about describing the motion as an ion plasma oscillation.

The numerical hybrid-MD approach permits the definition of a local temperature. Hence, it provides better temporal and spatial resolution of $T_{\mathrm{i}}$ than the experimental optical probes. As was shown in [73,29], the ion temperature shows clear oscillations in space (i.e. as a function of the distance from the center of the plasma) and, in fact, a wavelike oscillation pattern emanating from the plasma center (Fig. 19).

From the experimental data, it is not straightforward to comment on the damping of the observed kinetic energy oscillations because even in Fig. 17, which provides an annularly-resolved, more-local probe of the system, the analysis still averages over the density variation along the z-axis of the plasma. This introduces dephasing because the ion temperature oscillates with the local plasma frequency. A phenomenological model was developed in [71 to describe the ion equilibration and kinetic energy oscillations in a manner that could extract information on damping. The main assumptions of this model are that ions execute damped harmonic motion at their local plasma oscillation frequency and Eq. (63) determines the equilibrium ion kinetic energy and the amplitude of oscillation around this value. Fits to the data using this model showed that the damping time is close to $1 / \omega_{\mathrm{p}, \mathrm{i}}$, and higher particle density and lower electron temperature give slightly stronger damping. Numerical studies

of homogeneous strongly coupled plasmas [80,29] suggest a similar damping time for $\Gamma_{\mathrm{i}} \geq 5$, with lower $\Gamma_{\mathrm{i}}$ leading to faster damping.

\subsection{Collective Electronic Plasma Modes}

Oscillation of the electron plasma, or more precisely of the electron density, is a fundamental collective plasma mode first described by Tonks and Langmuir [138]. Excitation of this mode in an early experiment [32] firmly established that the photoionized ultracold gas forms a plasma.

A straightforward way to resonantly excite this mode is with a radio-frequency (rf) electric field (Fig. 5). A small DC bias directs electrons that escape the plasma to a charged particle detector in order to measure the response. Typical electron signals from such an experiment [32] are shown in Fig. 20a. The plasma is created at $t=0$ and some electrons promptly leave the sample and arrive at the detector at about $1 \mu \mathrm{s}$, producing the first peak in the signal. The resulting excess positive charge in the plasma creates the Coulomb potential well that traps the remaining electrons [2]. As the plasma expands, the depth of the Coulomb well decreases, allowing the remaining electrons to leave the trap. This produces the broad peak at $\sim 25 \mu \mathrm{s}$. 

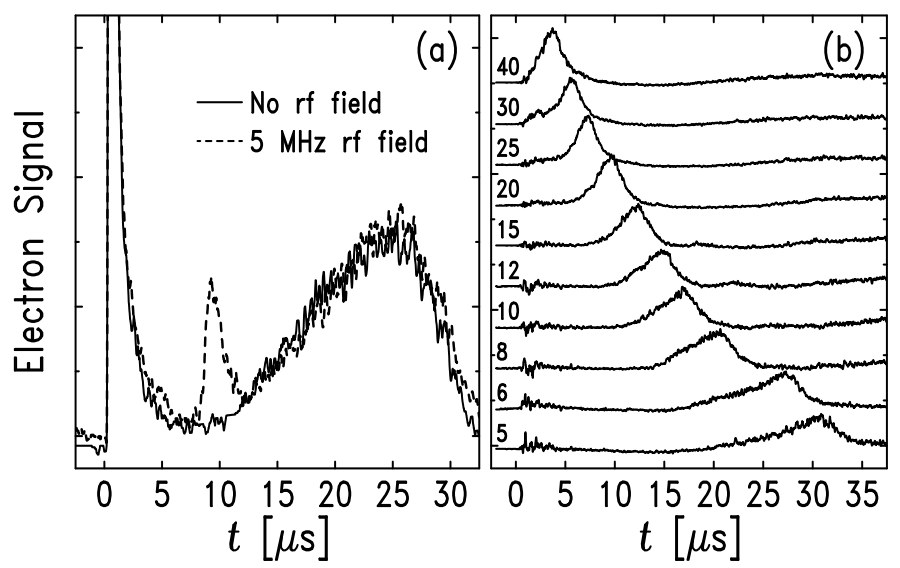

Fig. 20. Electron signals from ultracold xenon plasmas created by photoionization at $t=0$. (a) $3 \times 10^{4}$ atoms are photoionized and $E_{\mathrm{e}} / k_{\mathrm{B}}=540 \mathrm{~K}$. Signals with and without rf field are shown. The rf field is applied continuously. (b) $8 \times 10^{4}$ atoms are photoionized and $E_{\mathrm{e}} / k_{\mathrm{B}}=26 \mathrm{~K}$. For each trace, the rf frequency in $\mathrm{MHz}$ is indicated, and the nonresonant response has been subtracted. The signals have been offset for clarity. The resonant response arrives later for lower frequency, reflecting expansion of the plasma; Reused with permission from [32]. Copyright 2000, American Physical Society.

In the presence of an rf field an additional peak appears in the electron signal, indicating that resonant excitation of plasma oscillations has pumped energy into the plasma and raised the electron temperature. The elevated electron temperature causes more electrons to reach the detector because it increases the evaporation rate of electrons out of the Coulomb well.

This data 32] was initially analyzed assuming an expression for the angular oscillation frequency that is valid in a homogeneous gas $\left(\omega_{\mathrm{p}, \mathrm{e}}=\sqrt{e^{2} \rho_{\mathrm{e}} / \varepsilon_{0} m_{\mathrm{e}}}\right.$ [138]), and assuming that the excited mode was localized in regions of near resonant density. By assuming the peak response arrived when the average density in the plasma was in resonance, the changing resonant frequency showed the decrease in plasma density with time as the plasma expanded into the surrounding vacuum (Fig. 20b). Section 4.4 will discuss the expansion dynamics in detail.

Reference [49] pointed out that even in the zero-temperature limit, the electron plasma resonance frequency should be modified because of the inhomogeneous density distribution. This effect also causes the well known result that for a spherically symmetric flat-top density distribution, the frequency becomes $\omega=\omega_{\mathrm{p}, \mathrm{e}} / \sqrt{3}$. For a Gaussian density distribution, the plasma has a continuous spectrum of electron-density oscillation frequencies, but spectral weight is concentrated in a damped quasimode that is concentrated in lower density regions of the cloud and has a frequency between $1 / 4$ and $1 / 3$ of $\omega_{\text {p,e }}$ for the peak density in the distribution. This implies that the approach of 32 ] underestimated the density by about a factor of three, but does not change 


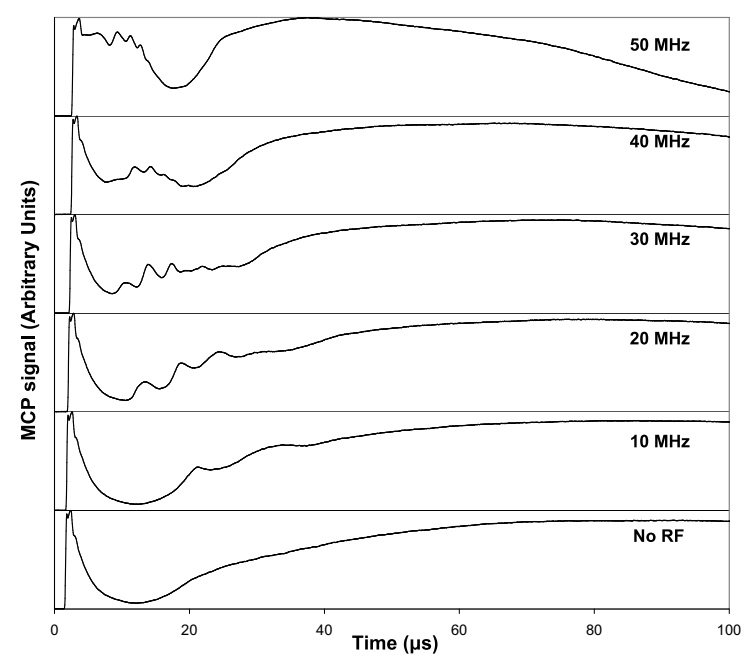

Fig. 21. Electron emission from an expanding ultracold plasma. Data is taken as in Fig. 20, but the plasma is more dense and the applied rf field is more intense. For a given rf frequency, the earliest rf response peak is the cold plasma mode at the frequency predicted by the theory of [49]. The later modes are thought to be Tonks-Dattner resonances [139]. Reused with permission from [50]. Copyright 2006, American Physical Society.

the general conclusion that the plasma oscillation can be used to follow the evolution of the plasma density.

In very recent work, this idea has been extended by Fletcher et al. [50] who observed what appear to be Tonks-Dattner modes, which are electron densitywaves characteristic of plasmas with inhomogeneous density distributions in which thermal effects are important. (See Fig. 21.) If one goes beyond cold plasma theory, and includes the effect of electron temperature, electron plasma oscillations follow the Bohm-Gross dispersion relation [140]

$$
\omega^{2}=\omega_{\mathrm{p}, \mathrm{e}}^{2}(r)+\frac{3 k_{\mathrm{B}} T_{\mathrm{e}}}{m_{\mathrm{e}}} k^{2}(r),
$$

where $k(r)$ is the local wavenumber. When the temperature or wavenumber becomes high, the thermal term becomes dominant and the electron density oscillation becomes a sound-wave. Tonks-Dattner modes are resonant electrondensity standing waves resulting from this thermal effect, and they were originally seen in cylindrical plasmas designed to simulate meteor tails [141,142]. In these experiments, the plasma density increased towards the central axis, 
and the modes resonated between the outer edge of the plasma and the region where $\omega=\omega_{\mathrm{p}, \mathrm{e}}$. For higher frequency modes, more wavelengths fit in the resonant region. It is at first surprising that thermal effects are important in ultracold plasmas. But because of the small plasma size, the wavelength for density waves must be small and the wavevector must be large. This makes the thermal term in the Bohm-Gross dispersion relation (Eq. (64) ) comparable or larger than $\omega_{\mathrm{p}, \mathrm{e}}$.

An accurate quantitative description of Tonks-Dattner modes was given by [139], and it was adapted to explain the observations in ultracold plasmas. The agreement is striking in spite of the fact that the authors had to make a somewhat ad hoc assumption about the outer boundary for the standing wave since there is not a well-defined edge to a Gaussian-shaped ultracold plasma. With additional theory input to understand this point, Tonks-Dattner modes should provide an accurate diagnostic of the electron temperature with good temporal resolution.

Hence, both types of collective electron excitations can provide information on the time evolution of the plasma, its changing density and electron temperature, and may be used to monitor dynamical effects in the plasma expansion.

\subsection{Plasma Expansion}

\subsubsection{Physical Description}

In all experiments performed so far, the plasma is unconfined and expands into the surrounding vacuum. On a time scale of about $10 \mu \mathrm{s}$, the plasma changes in size noticeably, as shown with absorption images of strontium plasmas in Fig. 22.

As discussed in Section 4.1.1 in conjunction with the initial electron trapping, the local charge imbalance due to the finite electron temperature causes a confining space charge potential. This traps the electrons such that a quasineutral plasma state develops, but the potential experienced by the ions has opposite sign, which drives the expansion of the plasma at later times. In an alternative, but equivalent description, simple hydrodynamic arguments [32] show that the expansion can also be related to the thermal pressure of the electrons, since the hydrodynamic force per ion due to the electron thermal motion and plasma spatial gradient is given by (see eq.(21) )

$$
\mathbf{F}=-\frac{k_{\mathrm{B}} T_{\mathrm{e}}}{\rho_{\mathrm{i}}} \nabla \rho_{\mathrm{i}}=\frac{k_{\mathrm{B}} T_{\mathrm{e}}}{\sigma^{2}} \mathbf{r} .
$$

This yields the same results as found from collisionless plasma dynamics using 


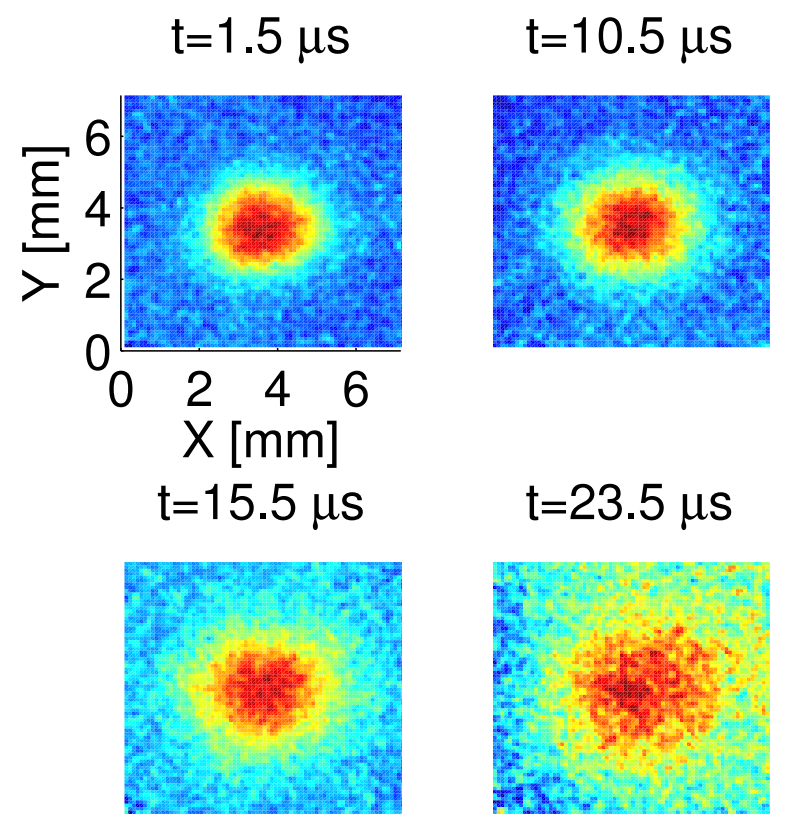

Fig. 22. False color images of expanding ultracold neutral strontium plasmas. The time after photoionization is indicated.

the Vlasov equation as described in Section 3.1 .13

This model predicts that the plasma phase space density retains its Gaussian shape (Eq. (22) ) during the expansion, with a quadratically increasing rms-size (Eq. 24a)

$$
\sigma(t)^{2}=\sigma(0)^{2}+v_{0}^{2} t^{2}
$$

where the expansion velocity $v_{0}=\sqrt{k_{\mathrm{B}} T_{\mathrm{e}}(0) / m_{i}}$ is solely determined by the initial electron temperature. The mechanical effect of the electrons on the ions is to induce a radially outward directed ion velocity (Eq. 26)

$$
\mathbf{u}(\mathbf{r}, t)=\frac{k_{\mathrm{B}} T_{\mathrm{e}}(0)}{m_{\mathrm{i}}} \frac{t}{\sigma(0)^{2}+v_{0}^{2} t^{2}} \mathbf{r}
$$

that is distinct from random thermal velocity, such that the ion kinetic energy due to expansion increases as $E_{\mathrm{i}}=\frac{3}{2} k_{\mathrm{B}} T_{\mathrm{e}}(0) v_{0}^{2} t^{2} / \sigma^{2}(t)$. This energy increase has to be compensated by a decreasing temperature of the electrons which cool according to $T_{\mathrm{e}}(t)=T_{\mathrm{e}}(0) \sigma^{2}(0) / \sigma^{2}(t)$ (Eq. $\left.24 \mathrm{~d}\right)$. Thus, asymptotically all the initial electron energy is transfered to the ions. Note that this energy transfer is not due to elastic collisions between the electrons and the ions, which would require milliseconds to equilibrate the energy of two plasma components.

The expansion of neutral plasmas in different geometries has been intensively studied for decades, and much theoretical work has recently been done on the

$\overline{3}$ Strong coupling of the ions [39] causes negligible modifications of the expansion dynamics (Section 3.2.3). 


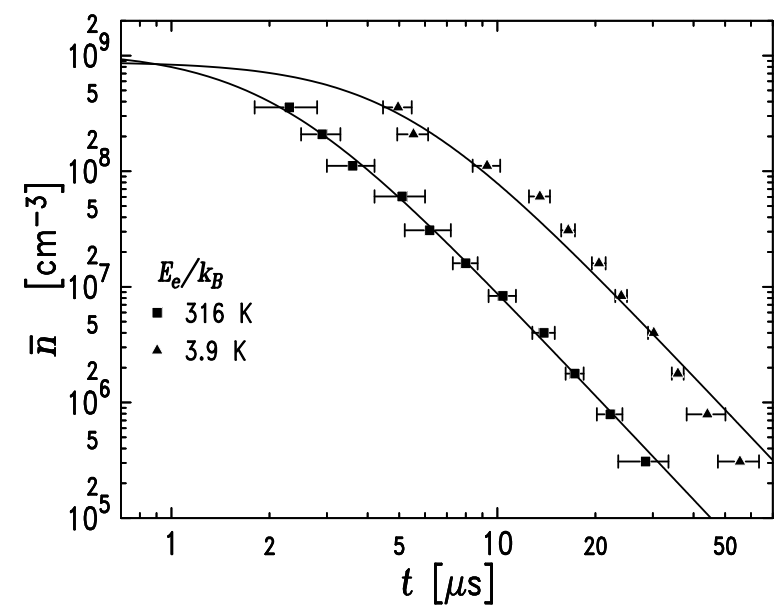

Fig. 23. Expansion of the plasma for $N=5 \times 10^{5}$ photoionized atoms. The expansion is described by $\bar{n}=N /\left[4 \pi\left(\sigma_{0}^{2}+v_{0}^{2} t^{2}\right)\right]^{3 / 2}$, where $\sigma_{0}$ is the initial rms radius, $v_{0}$ is the rms radial velocity at long times, and $\bar{n}$ is the density in resonance with the $\mathrm{rf}$ field, which is assumed to equal the average density in the plasma. Horizontal error bars arise from uncertainty in peak arrival times in data such as Fig. 20 b. The fits were consistently poor at low $E_{\mathrm{e}}$, as in the $3.9 \mathrm{~K}$ data. Reused with permission from [32]. Copyright 2000, American Physical Society.

dynamics of quasineutral plasmas expanding into a vacuum [92,143,144,145]. This problem is important for understanding plasmas created by irradiation of solid and thin film targets [146,17,16,19,20], clusters [25], and gas jets [27] by intense laser pulses, and dusty plasmas [147], which are important in astrophysical systems and plasma processing.

\subsubsection{Studying the Expansion with Electron Plasma Oscillations}

The first quantitative experimental study of the expansion was performed using excitation of electron plasma oscillations as a probe of the density as described in Section 4.3. Data such as in Fig. 23 was fitted assuming $\sigma(t)^{2}=$ $\sigma(0)^{2}+\left(v_{0} t\right)^{2}$ for the expansion. The oscillation probe is only sensitive to times when the plasma has already reached a final velocity. Figure 24] shows the extracted values of $v_{0}$. For data with $E_{\mathrm{e}} \geq 70 \mathrm{~K}$, the expansion velocities approximately follow $v_{0}=\left[E_{\mathrm{e}} /\left(\alpha m_{\mathrm{i}}\right)\right]^{1 / 2}$, where $m_{\mathrm{i}}$ is the ion mass and $\alpha=1.7$ is a fit parameter. This equates to $v_{0}=\left[0.9 k_{B} T_{\mathrm{e}}(0) / m_{\mathrm{i}}\right]^{1 / 2}$ if $k_{B} T_{\mathrm{e}}(0)=\frac{2}{3} E_{\mathrm{e}}$, in reasonable agreement with the theory.

For very low $E_{\mathrm{e}}$, the plasma expands as if the electrons had much more energy than $E_{\mathrm{e}}$ (Fig. 24). Different authors addressed this issue in a series of theory papers pointing to the importance of continuum lowering [148, disorder-induced heating [37,38, and recombination 42 as sources of electron heating. A more detailed discussion of these issues is given in Section 4.5. The heating becomes important just as $\Gamma_{\mathrm{e}}$ is approaching one, which is interesting, as it might in- 


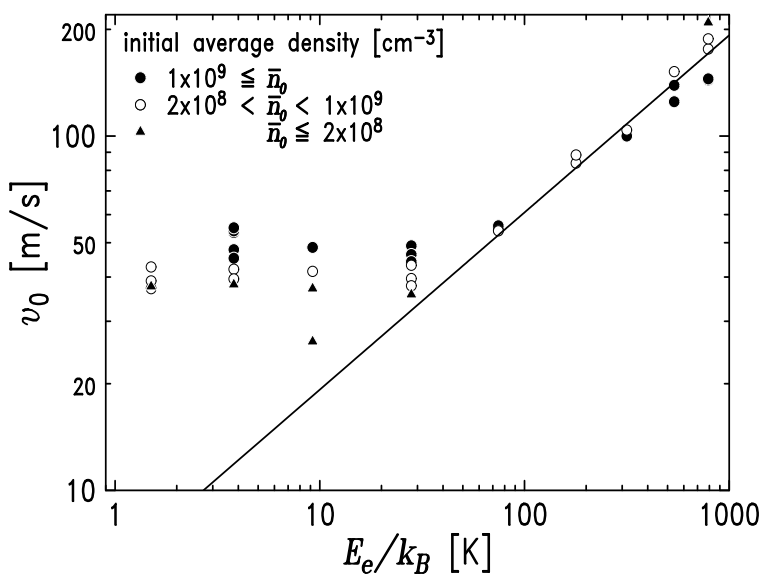

Fig. 24. Expansion velocities, $v_{0}$, found from fits to data such as in Fig. 23. The initial average density, $\bar{n}_{0}$, varies from $6 \times 10^{6}$ to $2.5 \times 10^{9} \mathrm{~cm}^{-3}$. The solid line, $v_{0}=\sqrt{E_{\mathrm{e}} / \alpha m_{\mathrm{i}}}$, with $\alpha=1.7$, is a fit to data with $E_{\mathrm{e}} / k_{B} \geq 70 \mathrm{~K}$. The behavior of low $E_{\mathrm{e}}$ data is discussed in the text. Uncertainty in $v_{0}$ is typically equal to the size of the symbols. There is a $0.5 \mathrm{~K}$ uncertainty in $E_{\mathrm{e}} / k_{B}$ reflecting uncertainty in the dye laser wavelength. Note that for $E_{\mathrm{e}} / k_{B}<70 \mathrm{~K}, v_{0}$ shows a systematic dependence on $\bar{n}_{0}$. Reused with permission from [32]. Copyright 2000, American Physical Society.

dicate the onset of strong coupling effects of the electrons. On the other hand this heating also implies that it is difficult to create strongly coupled electrons. There is still more work to be done to study the dependence of the heating on various parameters in order to work out the details of the dominant heating sources.

\subsubsection{Studying the Expansion with Optical Probes}

The expansion can also be studied with optical probes. Because of the better time resolution of these techniques, it is possible to follow the entire expansion from plasma formation, through ion acceleration, to terminal velocity. One can thus make an accurate comparison between experiment and theory.

References [70] and [13] used the ion fluorescence to follow the spatial expansion of a calcium plasma. By placing the focused excitation laser at various distances from the center of the plasma, they measured fluorescence profiles up to $50 \mu$ s after photoionization (Fig. 1 of [13]) and found reasonable agreement with a modified version of the theory [33,73] that addressed the lack of spherical symmetry. They also found that for $E_{e}<100 \mathrm{~K}$, the plasma expanded with more energy than expected from $E_{e}[70]$. The ion velocity was probed spectrally using fluorescence from a resonant excitation beam passing through the plasma center [13]. Changes in the fluorescence level could be ascribed to changes in Doppler broadening and ion velocity, resulting in the data in Fig. 18. 


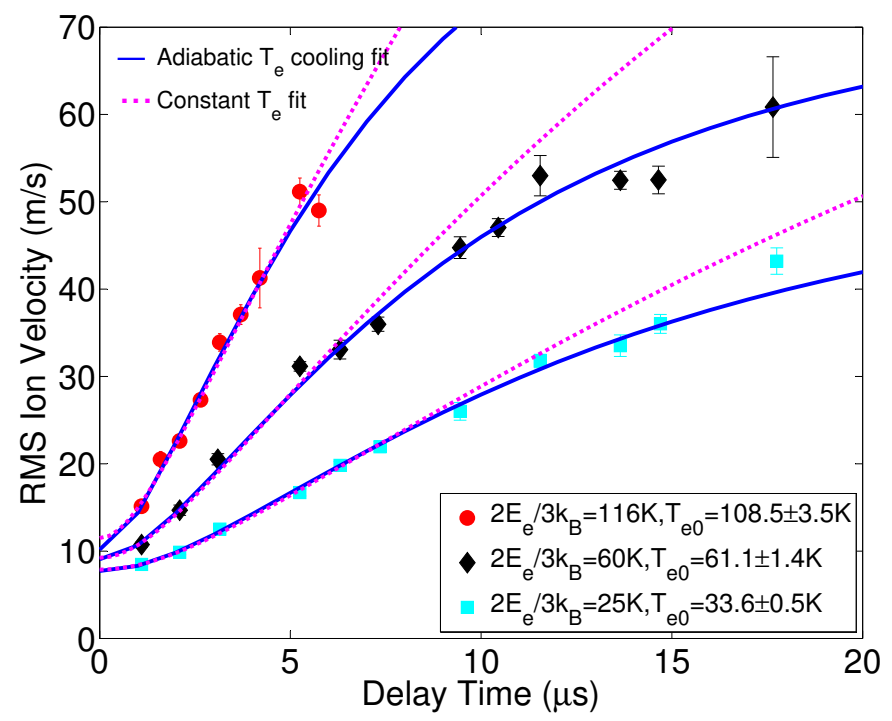

Fig. 25. Studying plasma expansion with optical absorption imaging. The rms velocity along the laser beam is extracted from the width of the absorption image (2.2.2). The dominant contribution is from the expansion velocity but there is a contribution from the ion thermal velocity that produces the $10 \mathrm{~m} / \mathrm{s}$ offset at short times. The initial peak density of these plasmas was $n_{0}=3.5 \times 10^{15} \mathrm{~m}^{-3}$ and initial size $\sigma_{0}=1 \mathrm{~mm}$. The initial electron kinetic energies are indicated in the legend. The solid-line fits to the data assume a self-similar expansion of the plasma as described in Eq. 67. This implies that the 1-dimensional $\mathrm{rms}$ ion velocity is given by $\sqrt{k_{B} T_{\mathrm{i}, \text { eff }} / m_{i}}$, where $T_{i, e f f}$ is given by Eq. (B.4). The initial electron temperature can be extracted from the fits, and the agreement with expected values for the two higher temperature plasmas indicates that three-body recombination and disorder-induced heating of electrons is not significant for this data. If the electron temperature did not drop due to adiabatic expansion, the ion velocity would follow the dashed-line curves.

In [28], the ion kinetic energy was found with the absorption spectra, and this was related to the expansion velocity and the average ion temperature. Initially, the technique was only applied to early times before adiabatic cooling of the electrons became important, but recent improvements have allowed study of longer times (Fig. 25). For a broad range of conditions, agreement is excellent with Eq. (B.4), which assumes a self-similar expansion (Eq. 24) and that after the first few microseconds the only mechanism effecting the electron temperature is adiabatic cooling. At lower $E_{e}$ and higher density, similar measurements show that electron heating effects contribute more significantly and for longer times (Section 4.5.2). The data shows strong indications of the approach to a terminal velocity, connecting the acceleration phase to the measurements of Figs. 23 and 24. 

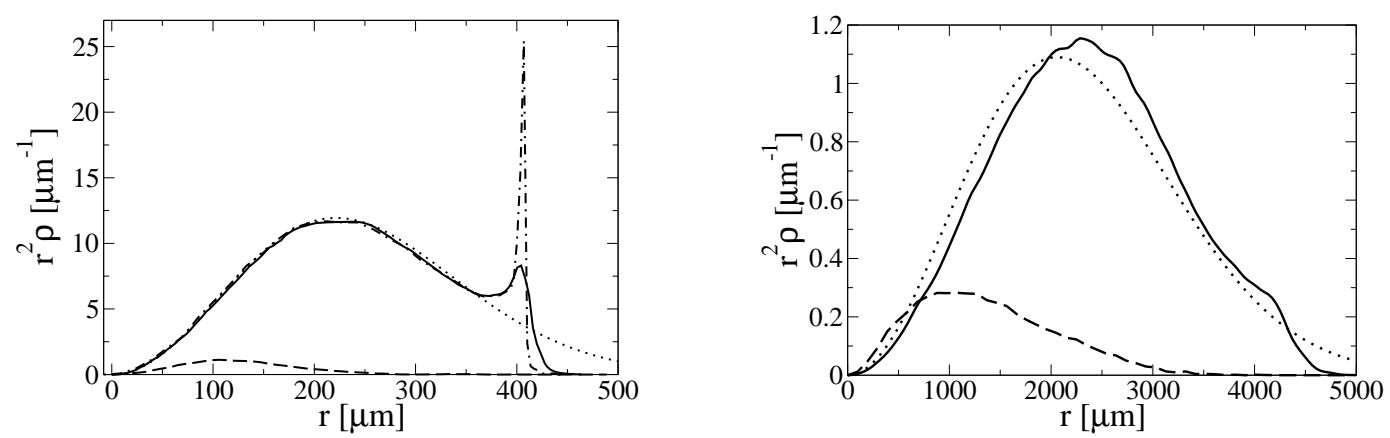

Fig. 26. Spatial densities $\rho_{\mathrm{i}}$ (solid) and $\rho_{\mathrm{a}}$ (dashed) of the ions and recombined atoms, respectively, at $t=3 \mu \mathrm{s}$ (a) and $t=31.3 \mu \mathrm{s}$ (b), compared to the Gaussian profile assumed for the hydrodynamical model (dotted). Additionally, $\rho_{\mathrm{i}}$ obtained from the particle simulation using the mean-field interaction only is shown as the dot-dashed line in (a). Reused with permission from [73]. Copyright 2004, American Physical Society.

\subsubsection{Plasma expansion and ion dynamics}

The effect of the plasma expansion on the ionic temperature and, in turn, the influence of the ion relaxation on the expansion, can be understood qualitatively from the hydrodynamical description of Section 3.1. First, neglecting ionic correlations $\left(U_{\mathrm{ii}}=0\right)$ the expansion leads to adiabatic cooling of the ions, analogous to the electron dynamics (see Eq. (24)). In addition, however, the ion temperature also changes due to the development of ionic correlations mainly through disorder-induced heating discussed in Section 4.2.1 and described by eqs. (39). Moreover, these correlations reduce the ion-ion interaction (compared to the spatially uncorrelated case, where pairs of closely neighboring atoms carry a large potential energy), and therefore lead to an effective negative acceleration, expressed by the $U_{\text {ii }} / 3$-term in eq. (39b), in addition to the ideal thermal pressure. This contribution, which corresponds to the average nonideal pressure known from homogeneous systems [3],97, also leads to an effective potential in which the ions move. As they expand in this potential, the thermal energy increases due to energy conservation. Finally, (39b) shows that the expansion is to the largest extent driven by the electronic pressure, since $T_{\mathrm{e}} \gg T_{\mathrm{i}}$ even after the disorder-induced heating, since $U_{\text {ii }}$ is of the same order as $T_{\mathrm{i}}$.

As the plasma expands, the spatial profile of the ions must deviate from its original Gaussian shape [33. This is mainly due to deviations from quasineutrality, e.g., deviations from the linear space dependence of the outward directed acceleration, at the plasma edge. The influence of the nonlinear correlation pressure on the density profile is of minor importance, as can be seen by comparing the solid and dot-dashed line of Fig. 26a in the inner plasma region. As known from earlier studies of expanding plasmas based on a meanfield treatment of the particle interactions [149,150,33], a sharp spike develops at the plasma edge, shown by the dot-dashed line in Fig. 26a. At later times, 


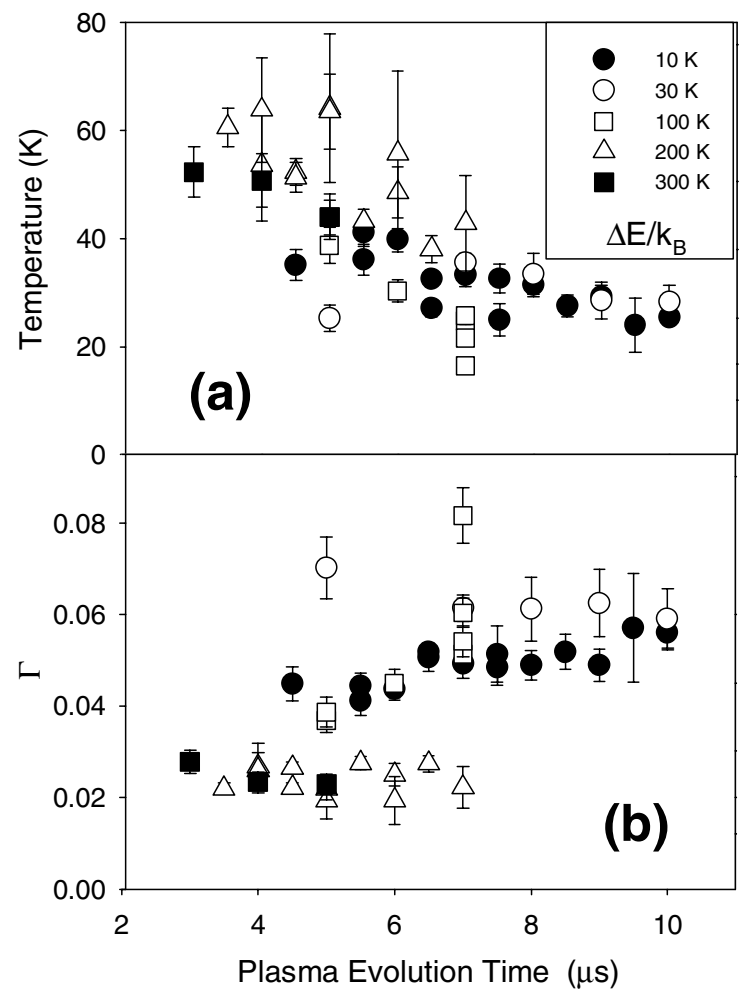

Fig. 27. Measured time evolution of the electron temperature (a) and electronic Coulomb coupling parameter (b) for initial ion numbers of $\sim 10^{6}$, initial plasma sizes of $0.4 \mathrm{~mm} \leq \sigma \leq 0.7 \mathrm{~mm}$ and different initial electron temperatures. Reused with permission from [67]. Copyright 2004, American Physical Society.

this spike decays again when the maximum of the hydrodynamic ion velocity passes the position of the density peak, so that the region of the peak is depleted. Ultimately, at long times, the entire plasma approaches the profile of a quasineutral selfsimilar expansion [150]. From Fig. 26a it becomes apparent that with ionic correlations, the peak structure is less pronounced than in mean field approximation. This is due to dissipation caused by ion-ion collisions which are fully taken into account in the hybrid-MD simulation. As shown in [150], by adding an ion viscosity term to the hydrodynamic equations of motion, dissipation tends to stabilize the ion density and prevents the occurrence of wavebreaking which was found to be responsible for the diverging ion density at the plasma edge in the case of a dissipationless plasma expansion. Furthermore, the initial correlation heating of the ions largely increases the thermal ion velocities leading to a broadening of the peak structure compared to the zero-temperature case. 


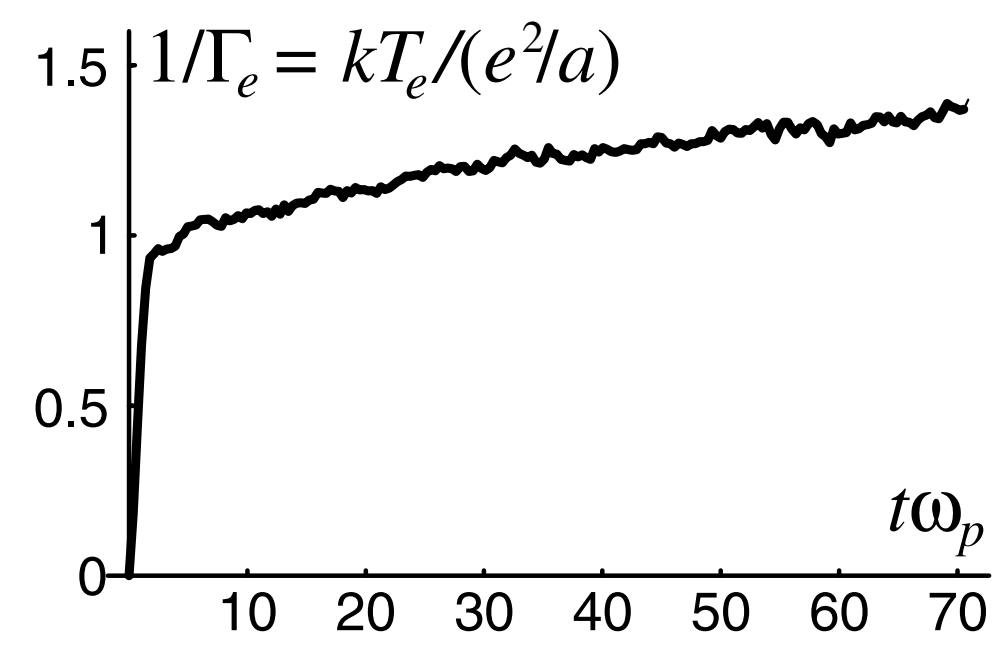

Fig. 28. Simulated time evolution of electron temperature scaled by the electron density with $T_{\mathrm{e}}(0)=0$. Reused with permission from [41]. Copyright 2002, American Physical Society.

\subsection{Electron Heating Mechanisms}

Figures 23 and 24 suggest that plasmas strongly deviate from the the simple selfsimilar expansion (Eq. (24) ) when the plasma becomes dense and the initial kinetic energy of the electrons, $E_{\mathrm{e}}$, becomes small, suggesting that additional physical processes heat the electrons. This question has been addressed in a series of theory papers [43, 148,38, 127, 41,37, 42,33,151], proposing different heating mechanisms. The general conclusion is that the electron heating processes restrict the temperature of the electrons such that the electron Coulomb Coupling parameter is limited to $\Gamma_{\mathrm{e}} \lesssim 0.2$.

The NIST group [67] determined the electron temperature directly as a function of time after photoionization by tipping the confining Coulomb potential and measuring the fraction of electrons that escaped over the lowered barrier. The potential was tipped by applying a small electric field pulse. The result in Fig. 27 shows that over a wide range of initial electron kinetic energies, $E_{\mathrm{e}}$, the electrons cooled or heated within a couple of microseconds to $T_{\mathrm{e}} \approx 60$ K. $T_{\mathrm{e}}$ subsequently dropped over the next $10 \mu \mathrm{s}$ along a similar curve for all conditions. While the high-temperature data are consistent with the collisionless expansion (Eq. (24)), the low-temperature results clearly indicate electron heating as the final temperature is well above the initial kinetic energy given to the electrons. 


\subsubsection{Disorder-induced electron heating}

While the temperature measurements of [67] were limited to times larger than a few microseconds due to the finite duration of the electric field pulse, detailed numerical studies of the short-time plasma dynamics have been performed by several groups [38,41,37]. Since an accurate molecular dynamics treatment requires huge numerical efforts, in [38,41,37] this aspect of the plasma dynamics has been studied for a smaller model system with a reduced ion-electron mass ratio. Fig. 28 shows the calculated time evolution of the scaled electron temperature $1 / \Gamma_{\mathrm{e}}$, for the extreme case of both zero initial ion temperature and zero initial electron kinetic energy, i.e., for an ionizing laser frequency that exactly matches the ionization potential of the cold atoms. Naively, one might expect very strong particle correlations at these low temperatures. However, the electron component is not created in a correlated equilibrium state and heats up in the same way as dicussed in Section 4.2 .1 for the ionic case. Immediately after photoionization spatial electron-electron and electron-ion correlations are practically negligible. They begin to develop on a timescale of the inverse electronic plasma frequency $\omega_{\mathrm{p}, \mathrm{e}}^{-1}=\sqrt{m_{\mathrm{e}} \varepsilon_{0} / e^{2} \rho_{\mathrm{e}}}$ lowering the electron potential energy, which is released as heat to the electrons. Due to the small electron mass the correlation build-up is much faster than for the ions, heating up the electrons within a few tens of nanoseconds. Since there are two contributions from electron-electron and from electron-ion correlations, we may estimate the final equilibrium excess correlation energy as two times the expression for $u_{\text {corr }}$ given in Eq. (43). Energy conservation, $3 \Gamma_{\mathrm{e}}^{-1} / 2=2 u_{\text {corr }}$, yields $\Gamma_{\mathrm{e}} \approx 1$, in agreement with the temperature dynamics shown in Fig. 28. An estimate of the released energy for the expansion velocity measurement of Fig. 24yields a plateau velocity of $13 \mathrm{~m} / \mathrm{s}$. The disorder induced heating alone is therefore not sufficient to explain the observed enhanced plasma expansion.

\subsubsection{Rydberg atoms formed through three-body recombination}

In three-body recombination (TBR) [152]44], an electron and an ion recombine to form a highly excited Rydberg atom, while a second electron participates in the collision to conserve energy and momentum. TBR is an important process in ultracold neutral plasmas, which may be surprising because at typical plasma temperatures of $1000 \mathrm{~K}$ or more, TBR is only important in dense plasmas. At low densities on the order of $10^{10} \mathrm{~cm}^{-3}$, recombination typically proceeds by radiative recombination (RR) and dielectronic recombination (DR) [152]153].

TBR dominates in ultracold plasmas because the total three-body rate varies with temperature as $T^{-9 / 2}$. This divergent temperature dependence has motivated investigations into whether TBR theory must be modified in the ultracold regime [154,155,156,43]. In addition to the fundamental interest in 


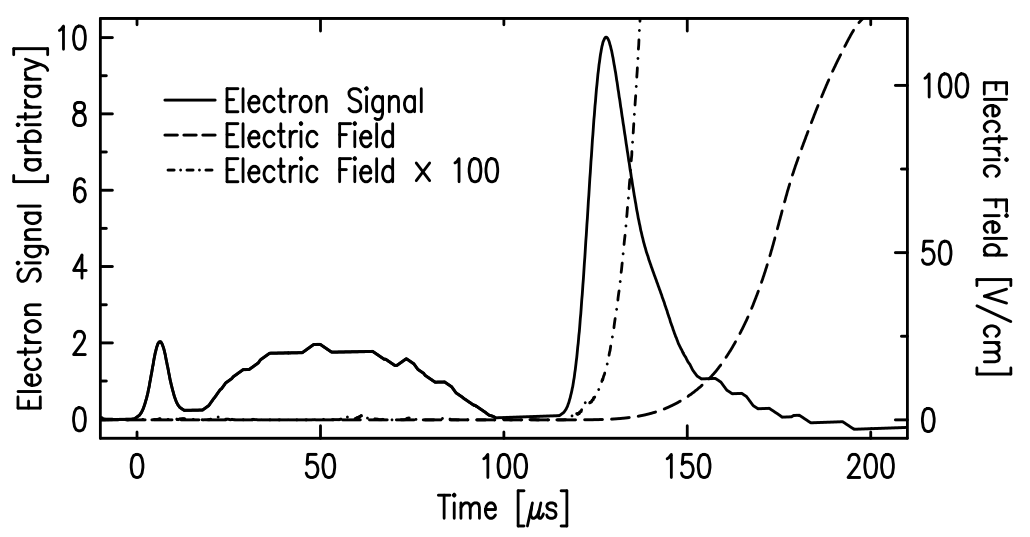

Fig. 29. Electron signal from a plasma created by photoionization of $10^{5}$ atoms at $t=0$, with $E_{\mathrm{e}} / k_{B}=206 \mathrm{~K}$. The first and second features represent free electrons escaping from the plasma. The third feature arises from ionization of Rydberg atoms. A field of $5 \mathrm{mV} / \mathrm{cm}$ is present before the large field ramp commences at about $120 \mu \mathrm{s}$. Electrons from the first two features are collected and detected with 10 times less efficiency than the Rydberg electrons. Reused with permission from [66]. Copyright 2001, American Physical Society.

TBR at low temperatures, the process is important in the formation of cold antihydrogen through positron-antiproton recombination [45,46]. In ion storage rings, collisions between ions and electrons in electron coolers can be in the $10-100 \mathrm{~K}$ energy range, and anomalously large recombination rates have been observed for highly charged ions [157]. Some authors have suggested an enhanced contribution from TBR to explain a portion of the excess 158 .

In addition to the fast initial rise of the temperature due to disorder-induced heating, Fig. 28 indicates further electron heating on a slower timescale, which is due to the formation of highly excited Rydberg atoms. Figure 29] shows experimental evidence of recombination in an ultracold neutral plasma. The origin of the time axis corresponds to the photoionization pulse. Immediately after that, some electrons leave the sample and are directed to the detector by a small DC field $(5 \mathrm{mV} / \mathrm{cm})$, producing the first peak in the signal. The ions are essentially immobile on this timescale, and the resulting excess positive charge in the plasma creates the Coulomb potential well that traps the remaining electrons [2]. The broad peak corresponds to the escape of free electrons as the Coulomb potential well vanishes as the plasma expands. After the free electrons have escaped, the electric field is increased to $120 \mathrm{~V} / \mathrm{cm}$ in $50-100 \mu \mathrm{s}$. This field can ionize Rydberg atoms bound by as much as $70 \mathrm{~K}$, corresponding to a principal quantum number of about $n=47$. From the number of electrons reaching the detector, the number of Rydberg atoms formed can be inferred, and from the field at which the atoms ionize the distribution of Rydberg atoms as a function of $n$ can be constructed. The Rydberg atoms survive for hundreds of microseconds with no significant change in their distribution. This implies that most of the atoms are in high angular momentum states which have long radiative lifetimes [58]. 

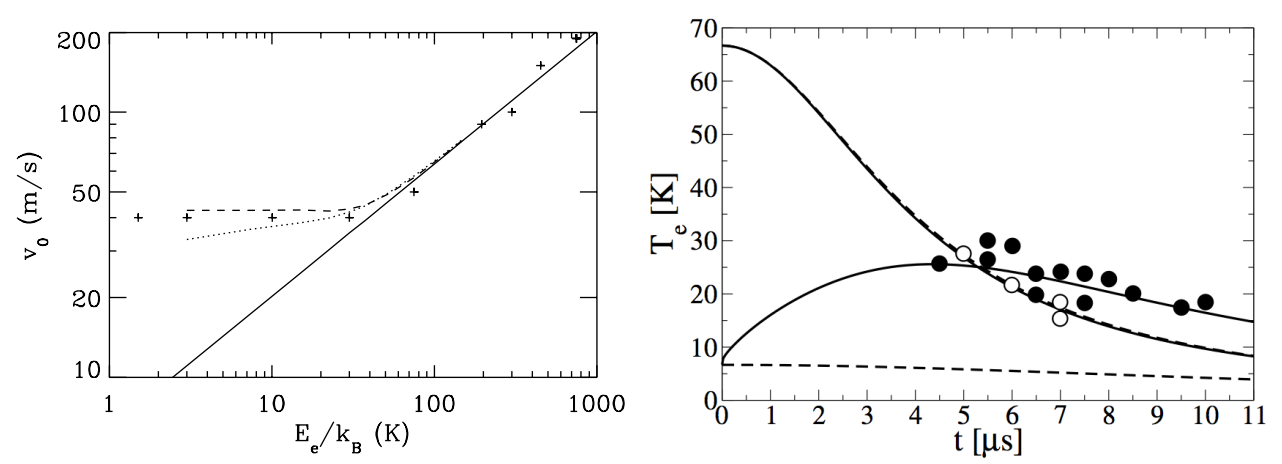

Fig. 30. (left): Comparison of PICMCC-calculations [42] and measurements [32] of the plasma expansion velocity. Initial state parameters are the same as in fig 24 . Reused with permission from [42]. Copyright 2002, American Physical Society. (right): Calculated time evolution of the electron temperature 29] compared to experiment 67] for two different initial electron temperatures of $6.7 \mathrm{~K}$ (filled circles) and 66.7 (open circles). The remaining parameters are the same as in fig.27, Reused with permission from [29]. Copyright 2005, Institute of Physics.

The classical three-body recombination theory, which is well confirmed for high temperature plasmas, predicts a total recombination rate of

$$
K_{\mathrm{tbr}}^{(\mathrm{tot})} \approx 3.8 \cdot 10^{-9} T_{\mathrm{e}}^{-9 / 2} \rho_{\mathrm{e}}^{2} s^{-1},
$$

where $T_{\mathrm{e}}$ is given in $\mathrm{K}$ and the density $\rho_{\mathrm{e}}$ is in $\mathrm{cm}^{-3}$. For typical parameters of $T=9 \mathrm{~K}$ and $\rho_{\mathrm{e}}=3 \cdot 10^{9} \mathrm{~cm}^{-1}$ this yields an extremely large recombination rate of $K_{\mathrm{tbr}}^{(\mathrm{tot})}=2.4 \cdot 10^{6} \mathrm{~s}^{-1}$. Hence, one would expect the plasma to almost completely convert into a Rydberg gas within a few microseconds. Contrary to this, only a few percent of recombination is observed after $12 \mu \mathrm{s}$ for the above initial conditions [66. This has led to a series of theoretical investigations questioning the validity of the standard recombination theory [43] and proposing strong coupling effects on the recombination rate [154 159]. Subsequent theory and experiments [66,37,42,67] indicate the opposite, namely that the plasma is driven out of the strongly coupled regime, by subsequent electron-atom collisions involving the formed Rydberg atoms, which tend to additionally heat the electron gas. The corresponding increase in temperature is in fact more rapid than from direct recombination and quickly quenches further recombination due to the strong $T_{\mathrm{e}}^{-9 / 2}$ dependence of the rate. The simple hydrodynamic description with standard collision rates [44, as described in Section 3.1.2, yields a surprisingly good description of the plasma dynamics. As shown in Fig. 30 the enhanced expansion velocity of Fig. 24 can indeed be reproduced upon inclusion of electron-Rydberg-atom collisions, and the hydrodynamic model even yields a quantitative description of the temperature measurement [67].

The measured distribution of principal quantum numbers for the Rydberg atoms also revealed that moderately tightly bound levels $(n<50)$ were populated in the plasma, and that the binding energy of the Rydberg atoms closely 
matched the excess energy that appeared in the expansion as shown in Fig. 24 [66].

Although the discussion about the validity of the conventional recombination theory under the conditions of an ultracold plasma is still not settled, the presented measurements [66,67] together with supporting theoretical calculations provide strong arguments for the applicability of the classical recombination rates even in the low -temperature regime.

\subsection{Coulomb Coupling Parameters}

As stated many times before in this review, fundamental interest in ultracold neutral plasmas to a large extent stems from the possibility of creating strongly coupled plasmas. For a system of charges embedded in a uniform neutralizing background, formally called a one-component plasma (OCP) [97, local spatial correlations characteristic of a strongly coupled fluid appear for $\Gamma \geq 2$. As $\Gamma$ further increases and eventually exceeds a value of 174 the system crystallizes. While the OCP model was originally developed to describe dense neutron stars, with ions embedded in a degenerate electron background, a clean experimental realization of an OCP is provided by laser cooled ion clouds confined in Penning [160,161,97] or Paul [162,163] traps.

In a real two-component plasma (TCP) the situation can be very different. Besides the possibility of forming bound states, electrons will tend to shield the ion-ion interaction potential. For weakly coupled electrons, within the DebyeHückel theory, the Coulomb interaction is replaced by an interaction potential $V_{\mathrm{ii}}=\exp \left(-r / \lambda_{\mathrm{D}}\right)$ of Yukawa-type. This shielding can drastically reduce the ion-ion interaction energy and the correlations, as approximately expressed by an effective coupling constant $\Gamma_{\mathrm{i}}^{\star}=\Gamma_{\mathrm{i}}^{-\kappa}$ [37]134], where $\kappa=a / \lambda_{\mathrm{D}}=\sqrt{3 \Gamma_{\mathrm{e}}}$. While this is certainly a good approximation for $\Gamma_{\mathrm{e}} \ll 1$ it breaks down as $\kappa>1$.

Ultracold plasmas provide the first precise experimental studies of two-component plasmas with both components in or near the strongly coupled regime. As we have discussed, however, various heating mechanisms currently preclude the exploration of plasmas deep in the strongly coupled regime $(\Gamma \gg 1)$.

\subsubsection{Electronic Coulomb Coupling Parameter}

At the lowest possible initial electron temperatures of about $0.1 \mathrm{~K}$, limited by the linewidth of the ionizing laser, and typical densities of $10^{9} \mathrm{~cm}^{-3}$ very large Coulomb parameters of about 30 might be expected. As outlined in the previous section there are however various heating mechanisms quickly decreas- 


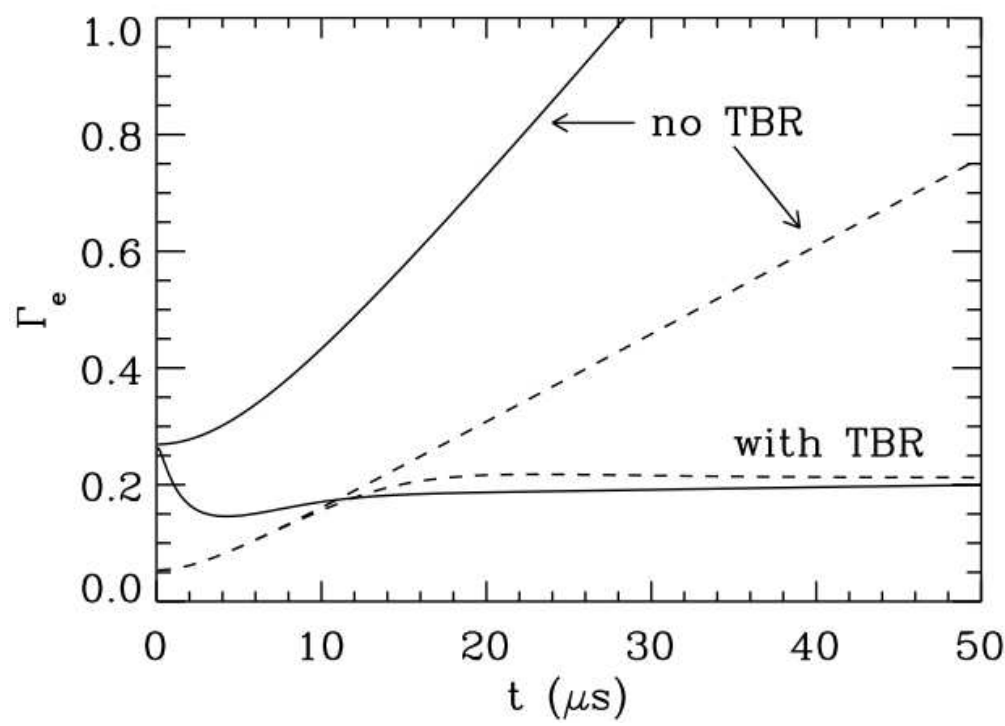

Fig. 31. Time evolution of the electron Coulomb coupling parameter for an initial average density of $10^{15} \mathrm{~cm}^{-3}$ and two different initial electron temperatures of $10 \mathrm{~K}$ (solid lines) and $50 \mathrm{~K}$ (dashed lines). Two simulations, including and excluding Rydberg atom formation are shown for each parameter set. Reused with permission from [42]. Copyright 2002, American Physical Society.

ing this value. After an initial disorder induced heating phase, the electrons are further heated by Rydberg atom formation and subsequent electron-atom collisions. Interestingly, $\Gamma_{\mathrm{e}}$ approaches an almost universal value largely independent of the initial plasma parameters. This is demonstrated in Fig. 31, showing results of PICMCC simulations (see Section 3.1) for two different initial temperatures [42]. When three-body recombination is turned off, $\Gamma_{\mathrm{e}}$ increases in time due to the adiabatic cooling of the electron gas. When the formation of Rydberg atoms is taken into account this increase is strongly suppressed by the heating of the electron gas. While the initial evolution is qualitatively different for the high and low temperature case, respectively, both curves approach a value of $\Gamma_{\mathrm{e}} \approx 0.2$ within about $10 \mu \mathrm{s}$. This prediction has been confirmed by subsequent temperature measurements, discussed in Section 4.5. Although such a PICMCC treatment can not describe any type of particle correlations of the plasma and hence neglect the disorder-induced electron heating, the measured value of the plateau is consistent with the theoretical result of 0.2 , within the experimental uncertainty. This suggests that Rydberg atom formation indeed constitutes the dominant heating mechanism and correlation effects do not play a significant role for the electron dynamics.

\subsubsection{Ionic Coulomb Coupling Parameter}

This situation is different for the ionic component, whose temperature is practically not affected by inelastic collisions due to the much larger mass of the ions. Heating can therefore only arise from correlation build-up. PICMCC 
simulations (Fig. 31) are unable to describe $\Gamma_{\mathrm{i}}$, since an accurate treatment of ion-ion correlations is required, as can be achieved within the hybrid-MD approach introduced in Section 3.2.3.

In order to calculate $\Gamma_{\mathrm{i}}$ one needs to know the ion temperature and density. Experimentally, $T_{\mathrm{i} \text {,ave }}$ can be determined from the absorption spectra [28] or ion fluorescence [13]. The density can be determined from absorption images, knowledge of the neutral atom parameters and the ionization fraction, or the timescale of the disorder-induced heating. In order to estimate the effect of screening, the electron temperature must be known. Finally, the inhomogeneity of the density distribution must be taken into account. One way to do this is to calculate the density-averaged coupling parameter, $\Gamma_{\mathrm{i}, \text { avg }}^{*}=\left\langle\exp [-\kappa(r)] e^{2} /\left[4 \pi \varepsilon_{0} a(r) k_{B} T_{\mathrm{i}, \text { ave }}\right]\right\rangle$. Measurements find for strontium plasmas $\Gamma_{\mathrm{i} \text {,avg }}^{*} \approx 2$ after the disorder-induced heating phase for a wide range of initial conditions [28]. With lower $T_{\mathrm{e}}$ and higher density, $\Gamma_{\mathrm{i}, \text { ave }}^{*}$ was only slightly higher. A similar result, $\Gamma \approx 4$, was found for calcium plasmas [13].

The surprisingly small variation in $\Gamma_{\mathrm{i} \text {,avg }}^{*}$ suggests that disorder-induced heating is a natural feedback mechanism that leads to equilibration into a state just barely in the strongly coupled fluid regime independent of the initial conditions. This conclusion is supported by the qualitative discussion on disorderinduced heating in Section 4.2.1, where it was argued that the final temperature should be of the order of the Coulomb interaction energy between neighboring ions, which directly translates into $\Gamma \approx 1$.

The question remains of how the Coulomb coupling parameter evolves in time as the system expands. The simple hydrodynamical model (Eq. 24) predicts that the ion temperature decrease during the expansion should overcompensate the accompanying decrease in density, so that the Coulomb coupling parameter should increase $\propto \rho^{-1 / 3}$ without bounds as the system expands. Experimentally, such a prediction is hard to check, since the directed radial expansion velocity quickly overwhelms the thermal velocity component. Theoretically, on the other hand, it is straightforward to separate the thermal velocity components in the hybrid-MD simulation [39].

Figure 32 shows that $\Gamma_{\mathrm{i}}$ does increase as the system expands. Hence, one might conclude that it is sufficient to simply wait long enough to allow the system to cool down in order to reach the crystallized state. However, it is questionable whether one should speak of a "crystal" if the density drops below that of interstellar space by the time it reaches a $\Gamma$ of 200 . But there is also a fundamental problem; the coupling parameter $\Gamma_{\mathrm{i}}$ measures the amount of correlations in a plasma if the system is in in thermodynamic equilibrium, but the plasma is created far from equilibrium in a completely uncorrelated initial state. Disorder-induced heating leads to local local thermal equilibrium, but the plasma continuously expands, so a true static equilibrium is never 


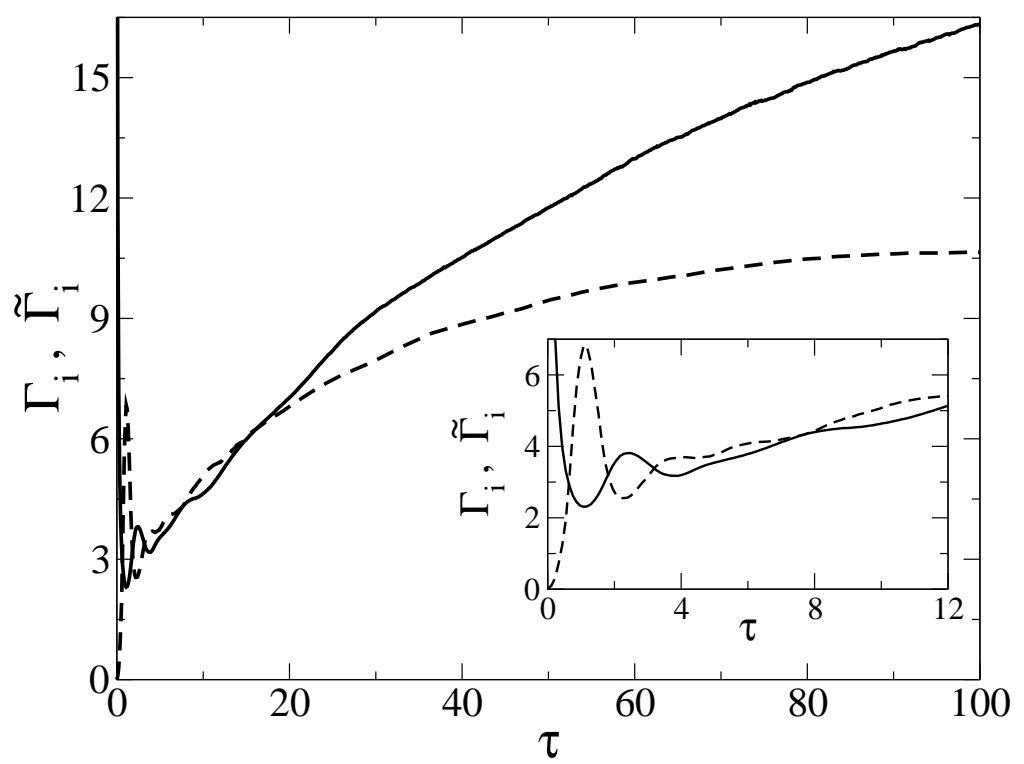

Fig. 32. Ionic Coulomb coupling parameter for a plasma with $N_{\mathrm{i}}(0)=5 \cdot 10^{4}$, $\bar{\rho}_{\mathrm{i}}(0)=1.1 \cdot 10^{9} \mathrm{~cm}^{-3}$ and $T_{\mathrm{e}}(0)=50 \mathrm{~K}$. The solid line shows the Coulomb coupling parameter calculated from the average temperature and density, the dashed line marks the coupling parameter extracted from pair correlation functions (see text). Inset: blow-up of the short-time behavior. Reused with permission from [30]. Copyright 2005, American Physical Society.

reached. One must compare the coupling parameter formally calculated from temperature and density with the spatial correlation properties of the system at all times.

In [30, this question was studied by extracting an alternative coupling parameter $\tilde{\Gamma}_{\mathrm{i}}$ from the pair correlation functions $g_{\mathrm{ii}}(r)$ obtained with the hybrid-MD approach (dashed line in Fig. 32). In line with the preceding discussion, $\Gamma_{i}$ and $\tilde{\Gamma}_{\mathrm{i}}$ initially have no similarity at all, reflecting the fact that the plasma is very far away from any kind of equilibrium. (In addition, Fig. 3(a) of reference [30] shows that the pair correlation function at early times is not well fit by that of an equilibrium system, so that a parametrization of $g_{\mathrm{ii}}$ by a single scalar quantity $\tilde{\Gamma}_{\mathrm{i}}$ does not adequately describe the plasma's spatial correlation properties. For $5<\omega_{\mathrm{p}, \mathrm{i}} t<20$, the two curves for $\Gamma_{\mathrm{i}}$ and $\tilde{\Gamma}_{\mathrm{i}}$ approach each other and run in parallel, suggesting that the plasma is close to a changing quasi-steady state during this stage.

For $\omega_{\mathrm{p}, \mathrm{i}} t>20$, however, the two curves diverge again, and $\tilde{\Gamma}_{\mathrm{i}}$ settles towards a finite value of $\tilde{\Gamma}_{\mathrm{i}} \approx 11$ deep in the strongly coupled regime, but still far away from a value where the occurrence of long-range order might be observed. In other words, while the system expands and cools down (reflected in the further increase of $\Gamma_{\mathrm{i}}$ ), the spatial correlations in the system (parametrized by $\tilde{\Gamma}_{\mathrm{i}}$ ) do not change anymore. On a formal level, this freeze-out of correlations is caused by another violation of the Bogoliubov assumption [136], namely a 
cross-over of the timescale for relaxation of correlations with the hydrodynamic timescale on which macroscopic system parameters change. More intuitively, the build-up of spatial correlations requires strong interactions between the ions in order to cause a rearrangement of their spatial correlational structure. As the system expands, the timescale on which such rearrangement takes place becomes longer and longer as the inter-ionic forces become weaker and weaker. At some point, the system simply becomes so dilute that the rearrangement does not occur anymore, and the spatial structure of the plasma freezes out in a selfsimilar expansion. As a consequence, the plasma remains in a state governed by strong short-range correlations, but no long-range order should form.

\section{$5 \quad$ Achieving Strong Coupling}

As emphasized in various sections of this article, one of the main motivations for studying ultracold plasmas from a plasma-physics perspective is the fact that they are strongly coupled, and one might hope to create crystalline plasmas. Coulomb crystallization is actively studied in one-component systems, such as magnetically confined ions [164,165] and dusty plasmas [166, 167,168]. Equivalent studies in two-component plasmas, on the other hand, are more scarce, as their experimental realization poses several additional constraints [169. In this respect ultracold neutral plasmas have attracted considerable attention as a promising opportunity to observe this phenomenon in the laboratory.

However, as discussed above, three-body recombination strongly heats the plasma electrons, while the disorder-induced heating rooted in the (spatially) uncorrelated nature of the initial plasma state (i.e., after creation of the plasma by photoionization) rapidly heats electrons and ions. This was realized soon after the first experiment, and different suggestions have been put forward to mitigate these effects.

For ions, these suggestions may be divided into two groups. On the one hand, one may avoid the disorder-induced heating. Since this heating effect is directly related to the uncorrelated initial state of the plasma, such approaches always aim at introducing some amount of correlation in the spatial distribution of the atoms before the plasma is formed by photoionization. We will discuss such approaches in Section 5.1 .2 below. On the other hand, one might counteract the disorder-induced heating by removing energy from the system during its evolution rather than avoiding the heating from the outset. In this case, one needs a way to couple the system to its environment in order to dissipate part of its internal energy, i.e., to cool it. Doppler laser cooling has been suggested as a way to achieve this goal. Since it is, in our opinion, the most promising 
perspective for achieving plasma crystallization at this point, we will discuss it in detail in Section 5.1.1. Finally, a recent proposal to increase the coupling of the plasma electrons by adding Rydberg atoms to the plasma, the only suggestion so far aiming at the electronic component of the plasma, will be briefly discussed in Section 5.2.

\subsection{Ionic coupling}

\subsubsection{Laser-cooling the ions}

As stated above, the idea behind laser cooling the plasma ions is to counteract disorder-induced heating by removing energy from the system via an external cooling mechanism. Such an approach is experimentally feasible if alkaline earth elements are used for the plasma, as their singly charged ions have a single outer electron which provides a convenient cooling transition for currently available laser systems. Hence, a "standard" Doppler cooling scheme may be used to cool the ions, with an optical molasses created by six counterpropagating lasers in a $3 \mathrm{~d}$ configuration (cf. Fig. 2). The plasma ions can then be cooled during the whole evolution of the system.

Experimentally, the scheme outlined above has not yet been realized. However, there is little doubt that it should be feasible 4 . In fact, a first step in this direction has already been taken with the Doppler imaging of the ion dynamics described in detail in Section 2.2.2, since the same transition that is used for the imaging can also be used for the cooling. Theoretically, the above scenario has been studied in detail in [34,94].

Originally, the idea of Doppler laser cooling the ions was proposed in [37] and [170]. These studies also correctly pointed out the potentially crucial role of elastic electron-ion collisions in the plasma in such a scenario. In a freely evolving plasma, such collisions are usually negligible, due to the fact that the timescale for electron-ion thermalization is much longer than the timescale of the plasma expansion. Hence, the heating of the ions during the first microsecond of evolution due to these collisions is limited to a few milli-Kelvin, which is negligible compared to the additional disorder-induced heating which is orders of magnitude stronger. In the case of laser cooling, however, the final ion temperature one is aiming for is also in the milli-Kelvin range, i.e., of the same order as the energy transferred from electrons to the ions via elastic collisions. Hence, these collisions should be taken into account when discussing the possibility of plasma crystallization. In [37,170], their influence on the achievable ionic temperature was estimated using reasonable values for initial-state

4 It should be noted, though, that the required cooling rates are more easily achieved for light ions such as Be. 
temperatures and densities. It was concluded that the ionic Coulomb coupling parameter would be limited to a value $\Gamma_{\mathrm{i}} \approx 50$ due to these elastic collisions, outside of the crystallized regime. However, these estimates assumed a constant heating rate corresponding to the chosen initial conditions. What was not taken into account is the expansion of the plasma, which strongly reduces the electron temperature and density, which reduces the collisional heating. As a consequence, these estimates are too pessimistic.

A detailed numerical investigation of the laser cooling process was carried out in [34.94], using both the hydrodynamic approach and the hybrid-MD approach introduced in Section 3. Insight into the modifications induced by the laser cooling can be obtained from a hydrodynamical description. On the level of a kinetic description, the influence of the cooling laser can be modeled via a Fokker-Planck term in the time evolution of the ionic distribution $f_{i}$,

$$
\left(\frac{\partial f_{\mathrm{i}}}{\partial t}\right)_{c}=\beta\left[\nabla_{\mathbf{v}}\left(\mathbf{v} f_{\mathrm{i}}\right)+\frac{k_{B} T_{D}}{m_{\mathrm{i}}} \Delta_{\mathbf{v}} f_{\mathrm{i}}\right],
$$

where $\beta$ is the cooling rate and $T_{D}$ the Doppler temperature. A derivation analogous to Section 3.1 leads to the macroscopic equations for the evolution,

$$
\begin{aligned}
\frac{\partial}{\partial t} \sigma^{2} & =2 \gamma \sigma^{2} \\
\frac{\partial}{\partial t} \gamma & =\frac{\left(k_{\mathrm{B}} T_{\mathrm{e}}+k_{\mathrm{B}} T_{\mathrm{i}}\right)}{m_{\mathrm{i}} \sigma^{2}}-\gamma(\gamma-\beta) \\
\frac{\partial}{\partial t}\left(k_{\mathrm{B}} T_{\mathrm{i}}\right) & =-2 \gamma k_{\mathrm{B}} T_{\mathrm{i}}-2 \beta\left(T_{\mathrm{i}}-T_{c}\right) \\
\frac{\partial}{\partial t}\left(k_{\mathrm{B}} T_{\mathrm{e}}\right) & =-2 \gamma k_{\mathrm{B}} T_{\mathrm{e}} .
\end{aligned}
$$

As readily observed from Eq. (70) the laser cooling dramatically modifies the expansion, not only quantitatively, but even qualitatively. While the width $\sigma$ of the plasma cloud increases linearly in time for the free expansion, it only varies as $\propto t^{1 / 4}$ in the laser-cooled case. As a consequence, the plasma expands much slowly than in the case of free expansion. This raises the hope of achieving a long-range ordered state, since we have seen in the previous section that it is the decrease in density and the corresponding increase in relaxation timescale that leads to a freeze-out of spatial correlation properties for a freely expanding plasma. In the laser-cooling scenario, the much slower decrease in density should allow for much longer times during which efficient rearrangement processes may occur, a prerequisite for the build-up of long-range order. One may, at first glance, think that this problem might be circumvented in any case by just increasing the cooling rate $\beta$ sufficiently. However, this is not the case, since a too rapid cooling could freeze the plasma in a metastable 

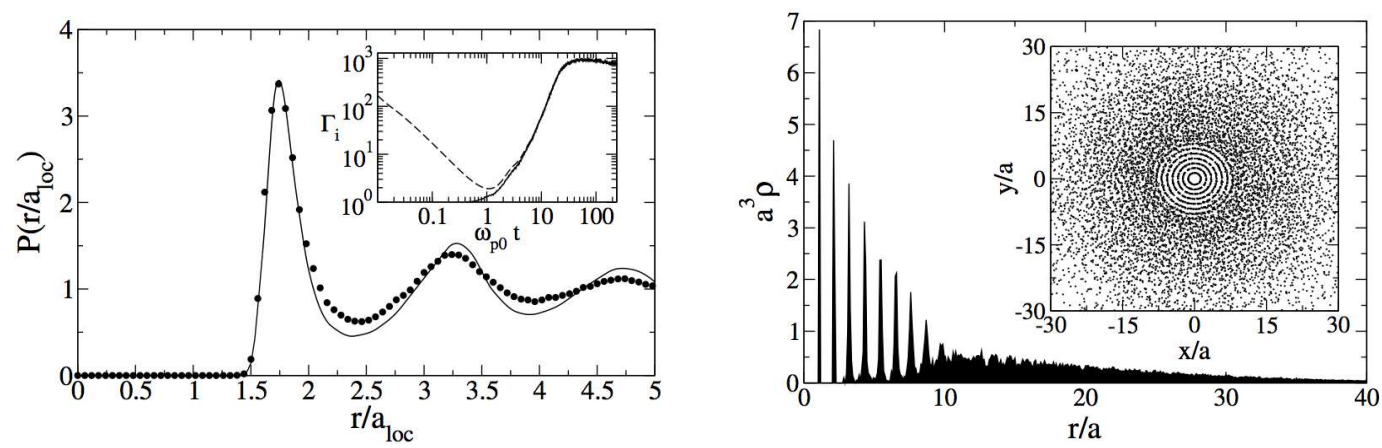

Fig. 33. Left: Distribution of scaled ion distances after an expansion time satisfying $\omega_{\mathrm{p}, \mathrm{i}} t=240$ (dots). The comparison with the correlation function of an OCP at $\Gamma_{\mathrm{i}}=700$ indicates the development of strong order. The inset shows the evolution of the ionic Coulomb coupling parameter determined from the correlation energy (solid line) and from the ion temperature and density (dashed line). Right: Shell structure formation during the plasma expansion demonstrated by the radial ion density and a two dimensional cut through the plasma. Reused with permission from 34]. Copyright 2004, American Physical Society.

disordered state. The system needs a certain time for spatial rearrangement to occur, which will not happen if the cooling is too fast.

In order to obtain information about the build-up of spatial correlations, one needs to go beyond a kinetic description of the ion distribution function. In 34 this was achieved within the hybrid-MD approach of Section 3.2.3, where the influence of the cooling lasers on the ions is described by a Langevin term consisting of a dissipative cooling force and a stochastic diffusive part. In addition, elastic electron-ion collisions should be taken into account in the description as discussed above. Such collisions can be implemented using a Monte Carlo procedure described in detail in [94]. It is found that the resulting structures formed during the system evolution may depend strongly on the initial-state parameters, such as number of ions, electron temperature, and cooling rate. Thus, the electronic temperature must be chosen sufficiently high in order to keep three-body recombination at a negligible level. As discussed in [34], three-body recombination creates doubly-excited and hence autoionizing states with an excess energy of several thousand Kelvin above the continuum limit. Hence, any autoionization process results in a fast electron which would not be bound in the ionic potential well, thereby destroying the plasma.

There are also significant constraints on the cooling rate $\beta$. As discussed above, if the cooling is too fast, the plasma will be frozen in a disordered glass-like state. On the other hand, if the cooling is too slow, the density decreases too strongly for effective inter-ionic interactions to take place. As we have seen in Section 4.6, reaching low temperatures and correspondingly high coupling parameters does not yet ensure spatial order in the plasma. However, it was demonstrated in [34,94] that, depending on particle number and cooling rate, 

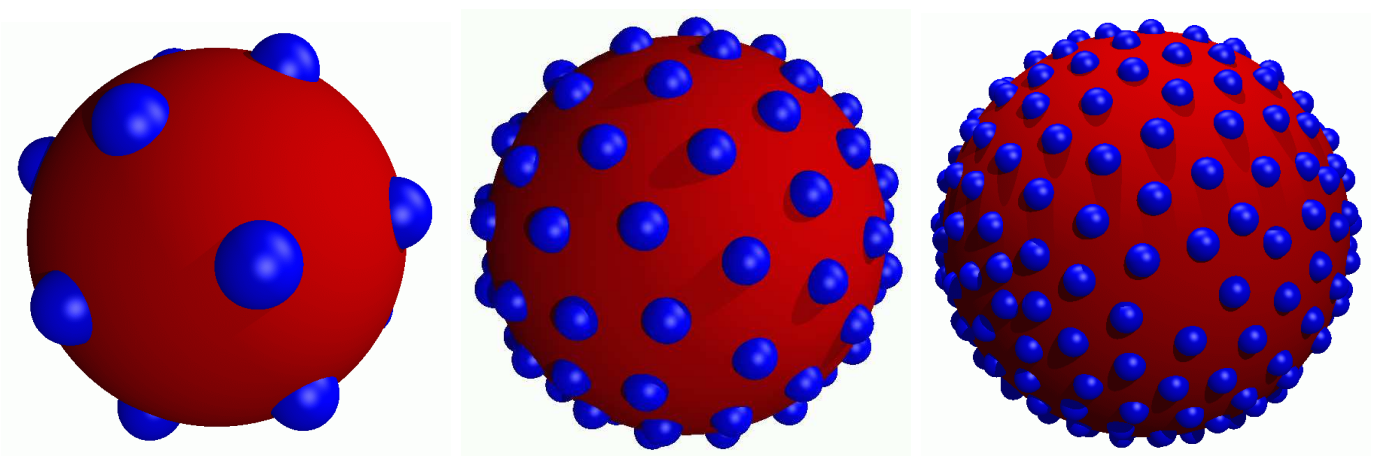

Fig. 34. Ionic intra-shell structure of the 1st, 3rd and 5th shell (from left to right). Reused with permission from [29], see also [34].

glass-like order or the formation of concentric shells may occur (see Fig. 33 and Fig, 34).

While the precise dynamics of this structure formation is not yet completely understood, it appears sufficiently robust that the crystallization should be observable experimentally with current state-of-the-art cooling techniques. Such an experimental confirmation, together with further detailed experimental and theoretical studies, would allow significant physical insight into the dynamics of strongly coupled two-component plasmas at the liquid-crystal phase transition. In particular, there are both similarities and differences with laser-cooled ion clouds in ion traps, where shell structures are also observed and intensively studied [160,171,172,173,174,175,176]. It may appear surprising that long-range order may occur in the present case of an unconfined plasma where, in contrast to trapped ion clouds, the crystallization process can only take place over a finite amount of time. Pronounced differences also exist in the dynamics of the crystallization process, which starts at the outer edge of the ion cloud in the case of ions in a trap, but is observed to proceed from the center of the plasma in the present scenario. Moreover, the spherical structure of small ion crystals can be attributed to the spherical symmetry of the trapping potential, while in the present case it must be traced to the spherical symmetry of the initial density distribution. This raises the question of what is the expected symmetry resulting from an ellipsoidal initial-state density [13.

\subsubsection{Other Approaches}

The crystallized regime would be reached more directly by avoiding the disorderinduced heating right from the start, rather than counteracting it by external cooling. Avoiding (or at least weakening) it requires a certain amount of spatial order in the initial state, i.e., one must generate a significant degree of spatial correlation before the plasma is created by photoionization. A number of proposals exist for how such an ordered state may be obtained, namely $(i)$ 
using fermionic atoms cooled below the Fermi temperature, so that the Fermi hole around each atom prevents the occurrence of small interatomic distances [40]; (ii) an intermediate step of exciting atoms into high Rydberg states, so that the interatomic spacing is at least twice the radius of the corresponding Rydberg state [103; and (iii) using optical lattices to arrange the atoms [103 133]. Calculations show that, indeed, these proposals could achieve a significant reduction of the disorder-induced heating, and the latter may even result in correlation-induced cooling of the plasma [133]. However, realizing the crystallized regime in this way turns to be very challenging experimentally and the laser-cooling approach discussed in the previous section seems to be more practical with current experimental capabilities.

\subsection{Electronic coupling}

All the scenarios discussed in the previous Section 5.1 aim at increasing the coupling of the ions in order to reach the crystallized regime. On the other hand, it would also be highly desirable to achieve strong coupling of the plasma electrons. Many previously employed assumptions, such as the dependence of three-body recombination rates on density and temperature, or the Thomson cut-off for the existence of Rydberg states are expected to fail in this regime. Moreover, this would provide an intriguing realization of a classical two-component system where both components are strongly coupled, with exciting prospects for observing new and interesting phenomena.

The only proposal so far targeting the electronic coupling parameter was introduced in [68]. The basic idea consists of deliberately adding Rydberg atoms to the plasma, which should then be collisionally ionized by the free plasma electrons, thereby reducing the kinetic energy of the electrons and cooling them. Such a scenario has been studied systematically in [177], and it was found that a moderate but still significant manipulation of the electron temperature is possible in this way. Figure 35 shows the time evolution of $T_{\mathrm{e}}$ for a plasma when Rydberg atoms with varying principal quantum number $n$ are added at $t=0$. As can be seen, adding Rydberg atoms with $n=36$ to the plasma leads to a significant reduction of $T_{\mathrm{e}}$ compared to the pure plasma without additional Rydberg atoms, in agreement with the original proposal [68. On the other hand, this effect strongly depends on the principal quantum number of the added Rydberg atoms. For lower excitation $(n=26$ in the present case), even the reverse effect of a heating of the plasma electrons rather than a cooling can be observed. This behavior, which might be surprising at first glance, is connected with the existence of a bottleneck in the transition rates for the inelastic electron-impact induced bound-bound and bound-free transitions of the atoms. If the binding energy $E_{\mathrm{b}}$ of an atom is larger than a critical (temperature-dependent) energy $E_{\mathrm{c}} \approx 4 k_{\mathrm{B}} T_{\mathrm{e}}$, the atom is predom- 


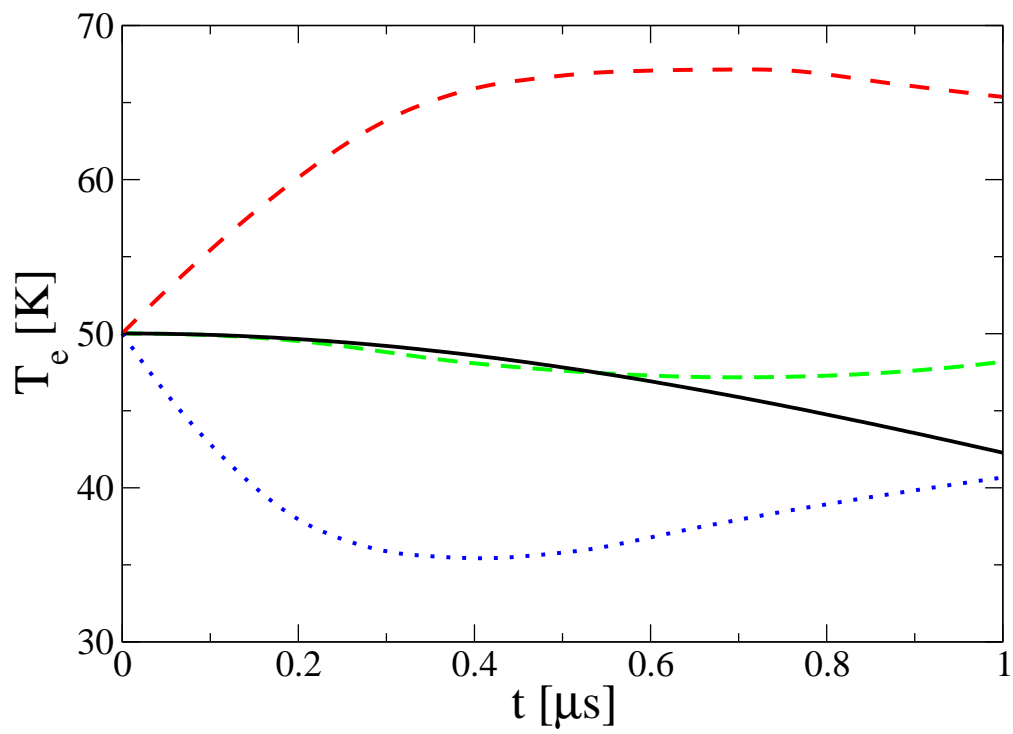

Fig. 35. Time evolution of $T_{\mathrm{e}}$ for a plasma of $N_{i}=80,000$ cesium ions with $T_{\mathrm{e}}(0)=50 \mathrm{~K}$ and $\sigma(0)=155 \mu \mathrm{m}$, after addition of $N_{\mathrm{R}}=80,000$ Rydberg atoms with principal quantum numbers $n=26,31,36$ (dashed lines), compared to that of the pure plasma without additional Rydberg atoms (solid line). Reused with permission from 68]. Copyright 2005, American Physical Society.

inantly driven to even lower states by collisions with the plasma electrons, thereby heating them. If, on the other hand, $E_{\mathrm{b}}$ is smaller than $E_{\mathrm{c}}$, collisions tend to excite the atom to higher bound states or ionize it, and the plasma electrons are cooled as originally envisioned.

Hence, by varying the principal quantum number of the added Rydberg atoms, it is possible to control the rate and even the sign of the change of the electron temperature. On the other hand, varying their number influences the absolute magnitude of the temperature change as well as the timescale over which cooling can be achieved. Together, a remarkable amount of control over the time evolution of the electronic temperature appears possible, in particular if one considers the possibility of more sophisticated schemes of adding the Rydberg atoms. Rather than adding all atoms simultaneously at $t=0$, one may think of adding them continuously over a certain amount of time, or in a number of individual pulses (or generally speaking "on demand"). One can also change the principal quantum number of the atoms in time. (The latter might be necessary, e.g., to stay away from the bottleneck separating cooling from heating, which changes as $T_{\mathrm{e}}$ changes in time.)

In addition to the temperature reduction discussed above, the collisional ionization of Rydberg atoms also increases the density of the plasma. Thus, both effects act cooperatively, increasing the Coulomb coupling parameter $\Gamma_{\mathrm{e}}$ as desired. It was found in [177] that, using relatively simple schemes for the addition of the atoms, $\Gamma_{\mathrm{e}}$ reaches values of $\Gamma_{\mathrm{e}} \approx 0.5$, as compared to $\Gamma_{\mathrm{e}} \lesssim 0.2$ 
found in the experiments with pure plasmas 5 . In more advanced scenarios as outlined above, reaching the strongly coupled state of $\Gamma_{\mathrm{e}}>1$ should most probably be realizable. The coupling strengths obtainable are certainly far away from the regime where a crystallization of the electrons might be observed. Still, pronounced liquid-like correlation effects may be anticipated, and the first realization of a two-component system where both the electronic and the ionic component are strongly coupled promises exciting new developments.

\section{Conclusions and Future Directions}

In this article we have reviewed the development in research on ultracold neutral plasmas since their first creation in 1999. We have described numerous experimental tools to probe various aspects of the system behavior, ranging from optical imaging of individual ions to employing collective plasma excitations as probes for macroscopic quantities. By combining such techniques, traditionally rooted in both atomic and plasma physics, these experiments have studied a number of plasma physics problems. They have revealed several surprises, which fosters continuing theoretical interest. We have outlined the theoretical concepts, developed to describe and understand the non-equilibrium dynamics of ultracold neutral plasmas. Our discussion has covered diverse approaches, such as simple reduced dynamical equations, kinetic or hydrodynamic descriptions and demanding microscopic approaches.

Based on the techniques introduced we have given a detailed description of the underlying physical processes driving this quite complex dynamical behavior. Within a hand-in-hand experimental and theoretical discussion a step-wise developed physical picture of the plasma dynamics over various evolution stages from its creation until its complete expansion into the surrounding vacuum has been given. Thereby we have covered a wide spectrum of phenomena, ranging from low-temperature collisions and Rydberg atom formation to the physics of collective plasma excitations and effects of strong correlations between the plasma constituents.

This wealth of interesting physics revealed in the past years demonstrates the rapid advancement in our understanding of these fascinating systems, but also raises various open questions, which we have pointed out in this article. Future studies in this young research field will certainly produce unexpected results, but several trends are likely to continue for the field.

5 Experimental results so far are consistent with this theoretical prediction, but still inconclusive since current schemes of extracting an electron temperature from the experiment are not very precise. 
As discussed in Section 5, the pursuit of stronger coupling in both the electrons and ions will remain an exciting and therefore intensively followed avenue of research. Experimental routes for increasing the ion coupling constant, such as laser-cooling [37,170] or photionizing a pre-ordered sample [40,133], may enable the formation of Wigner Crystals 34. This will hopefully allow exploration of the phase diagram of particles interacting through a screened Yukawa potential [178,101,102] that will compliment studies of dusty plasmas [9]. Creation of colder and denser electrons will show whether strongly coupled electrons can be realized in ultracold plasmas or three-body recombination precludes this possibility.

Exploring the regime of stronger electron coupling will necessarily probe the complicated evolution of the electron temperature when rapid thermalization, disorder-induced heating, threshold lowering, and three-body recombination play significant roles. Powerful theoretical tools have been developed to address these phenomena [41,37,38,42,33,43,73, but there is still need for a comprehensive study of evolution in various regimes of initial conditions and a disentangling of the many contributing effects. As emphasized in [33], the synthesis of ideas from atomic physics and plasma physics is crucial for this research. While techniques exist to study electron temperature at later times [67,50], at present there is no experimental diagnostic to cleanly explore the electron equilibration phase because electrons evolve so quickly. The development of such diagnostic would be a significant advance.

As the field grows, there is great possibility to increase the connection to traditional areas of plasma physics. The role of strong-coupling and the equilibration and expansion dynamics partly resemble the behavior of laser-produced plasmas in high energy-density experiments. But as we have emphasized from the start of this review, the slower time scales, excellent diagnostics, and control over initial conditions in ultracold plasmas provide unique opportunities to make significant contributions to these other areas.

The recent success to produce cold Rydberg gases and plasmas in strong magnetic fields of several Tesla [179,180] has opened up a new regime, where the atomic scale and macroscopic system dynamics is fundamentally different from the field-free situation. Theory has just started to treat collisional [181,106]107] and radiative [182 183,184] processes under such conditions, while the macroscopic dynamics of magnetized, ultracold, $\mu \mathrm{m}$-scale neutral plasmas still awaits a deeper understanding - all being essential questions for ongoing efforts to produce antihydrogen atoms [45,46].

New or improved experimental capabilities wait just over the horizon and will certainly open many avenues of research. As the resolution for imaging techniques approaches $\sim 10 \mu \mathrm{m}$, this will allow studies of collective modes or other features of the ion density distribution. The use of light scattering 
to directly observe spatial correlations [161] and the application of magnetic fields to confine the plasma will also provide new possibilities.

In light of the rapid development reviewed in this article, the study of ultracold neutral plasmas promises to remain an exciting and growing field of research for many years to come.

\section{A Important Quantities}

CCP - Coulomb Coupling Parameter

LTE - Local Thermal Equilibrium

MD - Molecular Dynamics

OCP - One Component Plasma

TCP - Two Component Plasma

PIC - Particle in Cell codes

PICMCC - Particle in Cell Monte Carlo Calculation

TBR - Three body recombination

$\mathrm{RR}$ - Radiative recombination

DR - Dielectronic recombination

* in all expressions $\alpha$ stands either for ions $(\alpha=\mathrm{i})$ or electrons $(\alpha=\mathrm{e})$

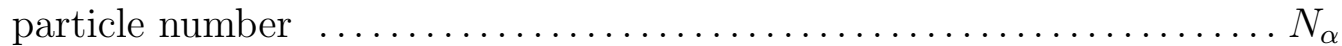

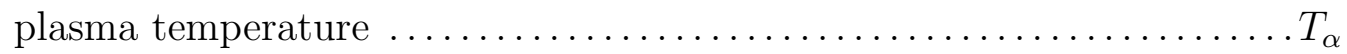

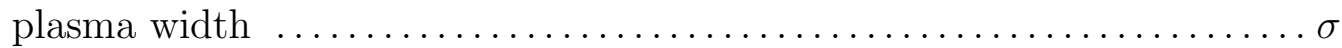

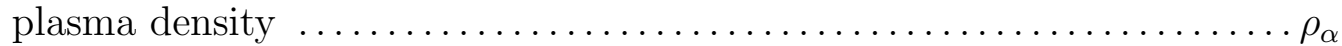
peak density $\ldots \ldots \ldots \ldots \ldots \ldots \ldots \ldots \ldots \ldots \ldots \rho_{0}=N_{\alpha} /\left(2 \pi \sigma^{2}\right)^{3 / 2}$

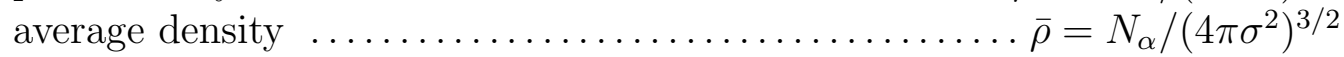
plasma frequency $\ldots \ldots \ldots \ldots \ldots \ldots \ldots \ldots \ldots \omega_{\mathrm{p}, \alpha}=\left[e^{2} \rho_{\alpha} /\left(m_{\alpha} \varepsilon_{0}\right)\right]^{1 / 2}$ Wigner-Seitz radius $\ldots \ldots \ldots \ldots \ldots \ldots \ldots \ldots \ldots \ldots a_{\alpha}=\left[3 /\left(4 \pi \rho_{\alpha}\right)\right]^{1 / 3}$

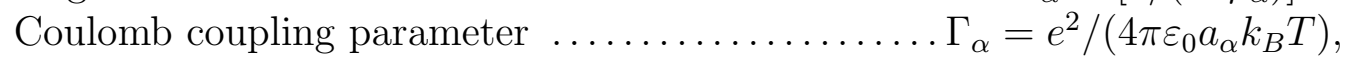

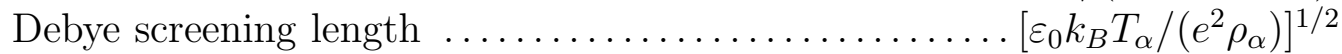
Brückner parameter $\ldots \ldots \ldots \ldots \ldots \ldots \ldots \ldots \ldots \ldots \ldots \ldots r_{s}=a_{\alpha} / a_{0}$ (ratio of interparticle distance to Bohr radius)

\section{B Extracting Plasma Parameters from the Absorption Spectrum}

The Voigt profile for the ion absorption cross section reads

$$
\alpha\left(\nu, T_{\mathrm{i}}\right)=\int d s \frac{3^{*} \lambda^{2}}{2 \pi} \frac{\gamma_{0}}{\gamma_{\mathrm{eff}}} \frac{1}{1+4\left(\frac{\nu-s}{\gamma_{\mathrm{eff}} / 2 \pi}\right)^{2}} \frac{1}{\sqrt{2 \pi} \sigma_{D}\left(T_{\mathrm{i}}\right)} \exp \left[-\frac{\left(s-\nu_{0}\right)^{2}}{2 \sigma_{D}\left(T_{\mathrm{i}}\right)^{2}}\right]
$$


where the parameters are described below Eq. (12) in Section 2.2.2.

The expansion of the plasma described in Section 4.4 eventually becomes the dominant source of Doppler broadening for the ion absorption spectrum in an ultracold neutral plasma (Fig. 7). Fortunately, the expansion is approximated well by a self-similar expansion of a Gaussian density distribution, and the dynamics takes a particularly simple form when there are no heat sources for the electrons and ion-electron thermalization is negligible. (See Section 3.1.2 and $32,33,34$.) These assumptions are reasonable for plasmas of low density and high initial electron energy $E_{e}$, although the exact regime of validity is the subject of current study. In this regime, the dynamics is determined by the parameters $T_{\mathrm{e}}(0)$ and $\sigma$, where $T_{\mathrm{e}}(0)$ is the electron temperature after any initial heating (Section 4.5). To be more specific, we assume that electron heating is only important at very early times before plasma expansion is important, and subsequently the electron temperature evolves only under the influence of the adiabatic expansion. Hence, $k_{B} T_{\mathrm{e}}(0)$ may be slightly higher than $\frac{2}{3} E_{\mathrm{e}}$.

The expansion velocity profile $\mathbf{u}(\mathbf{r}, t)$ (Eq. (26) ) gives rise to an average Doppler shift of the resonant frequency that varies with position,

$$
\delta \nu(\mathbf{r})=u_{z} / \lambda=\frac{r k_{B} T_{\mathrm{e}}(0) t}{m_{\mathrm{i}} \sigma^{2} \lambda} \cos \theta
$$

where $r \cos \theta$ is the displacement from the center of the cloud along the direction of laser propagation and $t$ is the time after photoionization. The positiondependent Doppler shift must be included in the exponent of the Gaussian describing the Doppler broadening in the Voigt convolution of the absorption cross section $\alpha\left(\nu, T_{\mathrm{i}}\right)$ (Eq. (B.1) $)$. The latter becomes explicitly position dependent and takes the form

$$
\begin{aligned}
& \alpha=\frac{3^{*} \lambda^{2}}{2 \pi} \frac{\gamma_{0}}{\gamma_{\mathrm{eff}}} F(\mathbf{r}) \\
& F(\mathbf{r})=\int d s \frac{1}{1+4\left(\frac{\nu-s}{\gamma_{\mathrm{eff}} / 2 \pi}\right)^{2}} \frac{1}{\sqrt{2 \pi} \sigma_{D}\left(T_{\mathrm{i}}(\mathbf{r})\right)} \exp \left[-\frac{\left(s-\nu_{0}-\delta \nu(\mathbf{r})\right)^{2}}{2 \sigma_{D}\left(T_{\mathrm{i}}(\mathbf{r})\right)^{2}}\right],
\end{aligned}
$$

where we have also included position dependence if the local thermal equilibrium temperature (Eq. (63) ). This variation of the ion temperature with position is the major complication to calculation of the full absorption $S(\nu)=$ $\int d^{3} r \rho_{\mathrm{i}}(\mathbf{r}) \alpha\left(\nu, T_{\mathrm{i}}, \mathbf{r}\right)$. But by replacing $T_{\mathrm{i}}(\mathbf{r})$ with the average ion temperature in the plasma, $T_{\mathrm{i} \text {,ave }}=\int d^{3} r T_{\mathrm{i}}(\mathbf{r})$ [54], and utilizing the spherical symmetry of the plasma, the integral over space can be evaluated, and the ion temperature can be replaced with an effective ion temperature $\left(T_{\mathrm{i}, \text { eff }}\right)$. The result is Eq. (12) in Section 2.2.2. One can analytically show that for a time $t$ after photoionization 


$$
T_{\mathrm{i}, \mathrm{eff}}=T_{\mathrm{i}, \text { ave }}+T_{\mathrm{e}}(0) t^{2} /\left(\tau_{\exp }^{2}+t^{2}\right) .
$$

The characteristic plasma expansion time is $\tau_{\exp }$ from Eq. (25). This enables a clear and well-defined analysis of the data, such as shown in Fig. 25. In principle, it also separates the various contributions to the Doppler broadening of the spectrum, although the expansion energy typically becomes an order of magnitude greater than the thermal energy, making quantitative measurement of the thermal energy difficult at later times. One can also consider comparison of Eqs. (12) and (B.4) with data as a check of the self-similar expansion model (Eq. (24)).

References [54] and [71] numerically justify approximate expressions relating $T_{\mathrm{i}, \mathrm{eff}}$ to $T_{\mathrm{i}, \text { ave }}, T_{\mathrm{e}}(0)$, time, and other physical parameters when the ion temperature is not assumed to be constant.

\section{The Quasineutral Approximation}

The assumption of quasineutrality constitutes a central part for the theoretical description and the analysis of ultracold plasma experiments. The condition of approximately equal electron and ion densities $\left(\rho_{\mathrm{e}} \approx \rho_{\mathrm{i}}\right)$ implies a well defined charge separation, but this fact often causes confusion and is rarely discussed in the literature. We provide a more detailed discussion of its physical meaning in this section.

We start our discussion with the set of Vlasov equations

$$
\begin{gathered}
\frac{\partial f_{\mathrm{e}}}{\partial t}+\mathbf{v}_{\mathrm{e}} \frac{\partial f_{\mathrm{e}}}{\partial \mathbf{r}_{\mathrm{e}}}-\frac{e}{m_{\mathrm{e}}} \frac{\partial f_{\mathrm{e}}}{\partial \mathbf{v}_{\mathrm{e}}} \frac{\partial \varphi}{\partial \mathbf{r}_{\mathrm{e}}}=0, \\
\frac{\partial f_{\mathrm{i}}}{\partial t}+\mathbf{v}_{\mathrm{i}} \frac{\partial f_{\mathrm{i}}}{\partial \mathbf{r}_{\mathrm{i}}}+\frac{e}{m_{\mathrm{i}}} \frac{\partial f_{\mathrm{i}}}{\partial \mathbf{v}_{\mathrm{i}}} \frac{\partial \varphi}{\partial \mathbf{r}_{\mathrm{i}}}=0,
\end{gathered}
$$

for the phase space distribution functions $f_{\alpha}\left(\mathbf{r}_{\alpha}, \mathbf{v}_{\alpha}\right)$, where $m_{\alpha}$ is the electron $(\alpha=\mathrm{e})$ and ion $(\alpha=\mathrm{i})$ mass and $\varphi$ is the total mean-field potential determined by the Poisson equation

$$
\Delta \varphi=\frac{e}{\varepsilon_{0}}\left(\rho_{\mathrm{e}}-\rho_{\mathrm{i}}\right),
$$

with $\rho_{\text {alpha }}=\int f_{\alpha} d \mathbf{v}_{\alpha}$. Most straightforwardly, the solution of these equations is obtained by calculating the potential $\varphi$ from Eq. (C.3) at every instant of time to propagate the phase space densities according to eqs.(C.1) and (C.2). However, the Vlasov equations could be used alternatively to calculate the electrostatic potential, while the Poisson equation determines the total charge density in this case. To do this we consider the hydrodynamic velocities

$$
\mathbf{u}_{\alpha}\left(\mathbf{r}_{\alpha}\right)=N_{\alpha}^{-1} \int \mathbf{v}_{\alpha} f_{\alpha}\left(\mathbf{r}_{\alpha}, \mathbf{v}_{\alpha}\right) d \mathbf{v}_{\alpha}
$$


whose time evolution is obtained by multiplying eqs. (C.1) and (C.2) by $\mathbf{v}_{\mathrm{e}}$ and $\mathbf{v}_{\mathbf{i}}$, respectively, and integrating over velocities. Upon subtracting of the resulting equations and using the continuity equations for the electron and ion densities we obtain for the electrostatic potential

$$
e \frac{\partial \varphi}{\partial \mathbf{r}}=\frac{\rho_{\mathrm{e}}\left(\frac{\partial \mathbf{u}_{\mathrm{e}}}{\partial t}+\mathbf{u}_{\mathrm{e}} \frac{\partial \mathbf{u}_{\mathrm{e}}}{\partial \mathbf{r}}\right)-\rho_{\mathrm{i}}\left(\frac{\partial \mathbf{u}_{\mathrm{i}}}{\partial t}+\mathbf{u}_{\mathrm{i}} \frac{\partial \mathbf{u}_{\mathrm{i}}}{\partial \mathbf{r}}\right)}{\rho_{\mathrm{e}} / m_{\mathrm{e}}+\rho_{\mathrm{i}} / m_{\mathrm{i}}}+\frac{\frac{\partial}{\partial \mathbf{r}}\left(\frac{\mathcal{P}_{\mathrm{e}}}{m_{\mathrm{e}}}-\frac{\mathcal{P}_{\mathrm{i}}}{m_{\mathrm{i}}}\right)}{\rho_{\mathrm{e}} / m_{\mathrm{e}}+\rho_{\mathrm{i}} / m_{\mathrm{i}}}
$$

For isotropic, thermal velocity distributions the pressure tensors $\mathcal{P}_{\alpha}$ become simple scalar fields given by $\mathcal{P}_{\alpha}=k_{\mathrm{B}} T_{\alpha} \rho_{\alpha}$. Since the time evolution of the hydrodynamic velocities and the pressure tensors follows from the self-consistent solution of the Vlasov equations this expression is completely equivalent to the Poisson equation (C.3). While the first term in Eq. (C.5) describes local charge imbalances between the two species, the second term arises solely from the thermal pressure of the particles. The latter only vanishes for the unlikely special case of $\mathcal{P}_{\mathrm{i}}=\frac{m_{\mathrm{i}}}{m_{\mathrm{e}}} \mathcal{P}_{\mathrm{e}}$, and, hence, yields a finite contribution to the electrostatic potential in general. On the other hand, the first can be neglected within the quasineutral approximation, requiring almost equal charge densities and local currents, i.e. $\rho_{\mathrm{i}} \approx \rho_{\mathrm{e}}$ and $\mathbf{u}_{\mathrm{i}}=\mathbf{u}_{\mathrm{e}}$. Thus the electrostatic potential can be calculated from

$$
e \frac{\partial \varphi}{\partial \mathbf{r}} \approx \frac{\frac{\partial}{\partial \mathbf{r}}\left(\frac{\mathcal{P}_{\mathrm{e}}}{m_{\mathrm{e}}}-\frac{\mathcal{P}_{\mathrm{i}}}{m_{\mathrm{i}}}\right)}{\rho_{\mathrm{e}} / m_{\mathrm{e}}+\rho_{\mathrm{i}} / m_{\mathrm{i}}} \approx k_{\mathrm{B}} T_{\mathrm{e}} \rho_{\mathrm{e}}^{-1} \frac{\partial \rho_{\mathrm{e}}}{\partial \mathbf{r}} \approx k_{\mathrm{B}} T_{\mathrm{e}} \rho_{\mathrm{i}}^{-1} \frac{\partial \rho_{\mathrm{i}}}{\partial \mathbf{r}}
$$

which coincides with Eq. (21), introduced in section 3.1.1. In the second step we have employed an adiabatic approximation for the electrons, making use of the relation $m_{\mathrm{e}} \ll m_{\mathrm{i}}$.

Note, that a direct application of the quasineutrality condition $\rho_{\mathrm{e}} \approx \rho_{\mathrm{i}}$ in the Poisson equation (C.3) would have resulted in a vanishing charge separation potential. The preceding discussion however shows that this is not the case since the thermal pressures of the plasma components generally yield a finite contribution of zero order in the charge separation. Rather the potential is given by Eq. (C.6), while the Poisson equation determines the separation of charge densities $\delta=\rho_{\mathrm{i}}-\rho_{\mathrm{e}}$ of the two species. However, we have $\delta / \rho_{\mathrm{e}} \ll 1$, ensuring quasineutrality and corresponding to the condition for the existence of a plasma state, introduced by Eq. (61).

\section{References}

[1] T. Pohl. Relaxation Dynamics of Ultracold Neutral Plasmas. PhD thesis, Technical University of Dresden, 2005. 
[2] T. C. Killian, S. Kulin, S. D. Bergeson, L. A. Orozco, C. Orzel, and S. L. Rolston. Creation of an ultracold neutral plasma. Phys. Rev. Lett., 83(23):4776, 1999.

[3] S. Ichimuru. Strongly coupled plasmas: high-density classical plasmas and degenerate electron liquids. Rev. Mod. Phys., 54(4):1017, 1982.

[4] H. M. Van Horn. Dense astrophysical plasmas. Science, 252:384, 1991.

[5] M. Nantel, G. Ma, S. Gu, C. Y. Cote, J. Itatani, and D. Umstadter. Pressure ionization and line merging in strongly coupled plasmas produced by 100 -fs laser pulses. Phys. Rev. Lett., 80(20):4442, 1998.

[6] E. Springate, N. Hay, J. W. G. Tisch, M. B. Mason, T. Ditmire, M. H. R. Hutchinson, and J. P. Marangos. Explosion of atomic clusters irradiated by high-intensity laser pulses: Scaling of ion energies with cluster and laser parameters. Phys. Rev. A, 61:063201, 2000.

[7] B. A. Remington. High energy density laboratory astrophysics. Plasma Physics and Controlled Fusion, 47:A191, October 2005.

[8] U. Saalmann, C. Siedschlag, and J.M. Rost. Mechanisms of cluster ionization in strong laser pulses. J. Phys. B, 39:R39, 2006.

[9] G. E. Morfill, H. M. Thomas, U. Konopka, and M. Zuzic. The plasma condensation: Liquid and crystalline plasmas. Phys. Plasmas, 6(5):1769, 1999.

[10] C. Hollenstein. Review article: The physics and chemistry of dusty plasmas. Plasma Physics and Controlled Fusion, 42:93, 2000.

[11] T. B. Mitchell, J. J. Bollinger, X. -P. Huang, W. M. Itano, and D. H. E. Dubin. Direct observations of the structural phases of crystallized ion plasmas. Phys. Plasmas, 6(5):1751, 1999.

[12] C. E. Simien, Y. C. Chen, P. Gupta, S. Laha, Y. N. Martinez, P. G. Mickelson, S. B. Nagel, and T. C. Killian. Using absorption imaging to study ion dynamics in an ultracold neutral plasma. Phys. Rev. Lett., 92(14):143001, 2004.

[13] E. A. Cummings, J. E. Daily, D. S. Durfee, and S. D. Bergeson. Fluorescence measurements of expanding strongly-coupled neutral plasmas. Phys. Rev. Lett, 95:235001, 2005.

[14] E. L. Clark, K. Krushelnick, M. Zepf, F. N. Beg, M. Tatarakis, A. Machacek, M. I. K. Santala, I. Watts, P. A. Norreys, and A. E. Dangor. Energetic heavyion and proton generation from ultraintense laser-plasma interactions with solids. Phys. Rev. Lett., 85(8):1654, 2000.

[15] R. A. Snavely, M. H. Key, S. P. Hatchett, T. E. Cowan, M. Roth, T. W. Phillips, M. A. Stoyer, E. A. Henry, T. C. Sangster, M. S. Singh, S. C. Wilks, A. MacKinnon, A. Offenberger, D. M. Pennington, K. Yasuike, A. B. Langdon, B. F. Lasinski, J. Johnson, M. D. Perry, and E. M. Campbell. Intense highenergy proton beams from petawatt-laser irradiation of solids. Phys. Rev. Lett., 85(14):2945, 2000. 
[16] S. P. Hatchett, C. G. Brown, T. E. Cowan, E. A. Henry, J. S. Johnson, M. H. Key, J. A. Koch, A. B. Langdon, B. F. Lasinski, R. W. Lee, A. J. Mackinnon, D. M. Pennington, M. D. Perry, T. W. Phillips, M. Roth, T. C. Sangster, M. S. Singh, R. A. Snavely, M. A. Stoyer, S. C. Wilks, and K. Yasuike. Electron, photon, and ion beams from the relativistic interaction of petawatt laser pulses with solid targets. In The 41st Annual Meeting of the Division of Plasma Physics of the American Physical Society, volume 7, page 2076. AIP, 2000.

[17] A. Maksimchuk, S. Gu, K. Flippo, D. Umstadter, and V. Yu. Bychenkov. Forward ion acceleration in thin films driven by a high-intensity laser. Phys. Rev. Lett., 84(18):4108, 2000.

[18] J. Badziak, E. Woryna, P. Parys, K. Yu. Platonov, S. Jabłoński, L. Ryć, A. B. Vankov, and J. Wołowski. Fast proton generation from ultrashort laser pulse interaction with double-layer foil targets. Phys. Rev. Lett., 87(21):215001, 2001.

[19] A. J. Mackinnon, Y. Sentoku, P. K. Patel, D. W. Price, S. Hatchett, M. H. Key, C. Andersen, R. Snavely, and R. R. Freeman. Enhancement of proton acceleration by hot-electron recirculation in thin foils irradiated by ultraintense laser pulses. Phys. Rev. Lett., 88(21):215006, 2002.

[20] M. Hegelich, S. Karsch, G. Pretzler, D. Habs, K. Witte, W. Guenther, M. Allen, A. Blazevic, J. Fuchs, J. C. Gauthier, M. Geissel, P. Audebert, T. Cowan, and M. Roth. MeV ion jets from short-pulse-laser interaction with thin foils. Phys. Rev. Lett., 89(8):085002, 2002.

[21] M. Allen, Y. Sentoku, P. Audebert, A. Blazevic, T. Cowan, J. Fuchs, J. C. Gauthie, M. Geissel, M. Hegelich, S. Karsch, E. Morse, P. K. Patel, and M. Roth. Proton spectra from ultraintense laser?plasma interaction with thin foils: Experiments, theory, and simulation. Phys. Plasmas, 10:3283, 2003.

[22] M. Kaluza, J. Schreiber, M. I. K. Santala, G. D. Tsakiris, K. Eidmann, J. Meyer-Ter-Vehn, and K. J. Witte. Influence of the laser prepulse on proton acceleration in thin-foil experiments. Phys. Rev. Lett., 93(4):045003, 2004.

[23] J. Fuchs, Y. Sentoku, S. Karsch, J. Cobble, P. Audebert, A. Kemp, A. Nikroo, P. Antici, E. Brambrink, A. Blazevic, E. M. Campbell, J. C. Fernndez, J.C. Gauthier, M. Geissel, M. Hegelich, H. Ppin, H. Popescu, N. Renard-LeGalloudec, M. Roth, J. Schreiber, R. Stephens, and T. E. Cowan. Comparison of laser ion acceleration from the front and rear surfaces of thin foils. Phys. Rev. Lett., 94:045004, 2005.

[24] L. Romagnani, J. Fuchs, M. Borghesi, P. Antici, P. Audebert, F. Ceccherini, T. Cowan, T. Grismayer, S. Kar, A. Macchi, P. Mora, G. Pretzler, A. Schiavi, T. Toncian, and O. Willi. Dynamics of electric fields driving the laser acceleration of multi-MeV protons. Phys. Rev. Lett., 95:195001, 2005.

[25] T. Ditmire, J. W. G. Tisch, E. Springate, M. B. Mason, N. Hay, R. A. Smith, J. Marangos, and M. H. R. Hutchinson. High-energy ions produced in explosions of superheated atomic clusters. Nature, 386:54, 1997. 
[26] G. S. Sarkisov, V. Yu. Bychenkov, V. N. Novikov, V. T. Tikhonchuk, A. Maksimchuk, S.-Y. Chen, R. Wagner, G. Mourou, and D. Umstadter. Selffocusing, channel formation, and high-energy ion generation in interaction of an intense short laser pulse with a He jet. Phys. Rev. E, 59(6):7042, 1999.

[27] K. Krushelnick, E. L. Clark, Z. Najmudin, M. Salvati, M. I. K. Santala, M. Tatarakis, A. E. Dangor, V. Malka, D. Neely, R. Allott, and C. Danson. Multi-MeV ion production from high-intensity laser interactions with underdense plasmas. Phys. Rev. Lett., 83(4):737, 1999.

[28] Y. C. Chen, C. E. Simien, S. Laha, P. Gupta, Y. N. Martinez, P. G. Mickelson, S. B. Nagel, and T. C. Killian. Electron screening and kinetic energy oscillations in a strongly coupled plasma. Phys. Rev. Lett., 93:265003, 2004.

[29] T. Pohl and T. Pattard. Strong-coupling effects in the relaxation dynamics of ultracold neutral plasmas. J. Phys. Conf. Series, 11:223, 2005.

[30] T. Pohl, T. Pattard, and J. M. Rost. Relaxation to non-equilibrium in expanding ultrcold neutral plasmas. Phys. Rev. Lett., 94:205003, 2005.

[31] M. S. Murillo. Ultrafast dynamics of strongly coupled plasmas. Phys. Rev. Lett., 96:165001, 2006.

[32] S. Kulin, T. C. Killian, S. D. Bergeson, and S. L. Rolston. Plasma oscillations and expansion of an ultracold neutral plasma. Phys. Rev. Lett., 85(2):318, 2000 .

[33] F. Robicheaux and J. D. Hanson. Simulated expansion of an ultra-cold, neutral plasma. Phys. Plasmas, 10(6):2217, 2003.

[34] T. Pohl, T. Pattard, and J. M. Rost. Coulomb crystallization in expanding laser-cooled neutral plasmas. Phys. Rev. Lett., 92(15):155003, 2004.

[35] J. Lindl. Development of the indirect-drive approach to intertial confinement fusion and the target physics basis for ignition and gain. Phys. Plasmas, 2:3933, 1995.

[36] H. Daido. Review of soft x-ray laser researches and developments. Rep. Prog. Phys., 65(10):1513, 2002.

[37] S. G. Kuzmin and T. M. O’Neil. Numerical simulation of ultracold plasmas. Phys. Plasmas, 9(9):3743, 2002.

[38] S. Mazevet, L. A. Collins, and J. D. Kress. Evolution of ultracold neutral plasmas. Phys. Rev. Lett., 88(5):55001, 2002.

[39] T. Pohl, T. Pattard, and J. M. Rost. Kinetic modeling and molecular dynamics simulation of ultracold neutral plasmas including ionic correlations. Phys. Rev. A, 70(3):033416, 2004.

[40] M. S. Murillo. Using Fermi statistics to create strongly coupled ion plasmas in atom traps. Phys. Rev. Lett., 87(11):115003, 2001. 
[41] S. G. Kuzmin and T. M. O'Neil. Numerical simulation of ultracold plasmas: How rapid intrinsic heating limits the development of correlation. Phys. Rev. Lett., 88(6):65003, 2002.

[42] F. Robicheaux and J. D. Hanson. Simulation of the expansion of an ultracold neutral plasma. Phys. Rev. Lett., 88(5):55002, 2002.

[43] A. N. Tkachev and S. I. Yakovlenko. Moderation of recombination in an ultracold laser-produced plasma. Quantum Electronics, 30(12):1077, 2000.

[44] P. Mansbach and J. Keck. Monte Carlo trajectory calculations of atomic excitation and ionization by thermal electrons. Phys. Rev., 181:275, 1969.

[45] M. Amoretti, C. Amsler, G. Bonomi, A. Bouchta, P. Bowe, C. Carraro, C. L. Cesar, M. Charlton, M. J. T. Collier, M. Doser, V. Filippini, K. S. Fine, A. Fontana, M. C. Fujiwara, R. Funakoshi, P. Genova, J. S. Hangst, R. S. Hayano, M. H. Holzscheiter, L. V. Jrgensen, V. Lagomarsino, R. Landua, D. Lindelf, E. Lodi Rizzini, M. Macr, N. Madsen, G. Manuzio, M. Marchesotti, P. Montagna, H. Pruys, C. Regenfus, P. Riedler, J. Rochet, A. Rotondi, G. Rouleau, G. Testera, A. Variola, T. L. Watson, and D. P. van der Werf. Production and detection of cold antihydrogen atoms. Nature, 419:456, 2002.

[46] G. Gabrielse, N. S. Bowden, P. Oxley, A. Speck, C. H. Storry, J. N. Tan, M. Wessels, D. Grzonka, W. Oelert, G. Schepers, T. Sefzick, J. Walz, H. Pittner, T. W. Hansch, and E. A. Hessels ATRAP Collaboration. Background-free observation of cold antihydrogen with field-ionization analysis of its states. Phys. Rev. Lett., 89(21):213401, 2002.

[47] D. Comparat, T. Vogt, N. Zahzam, M. Mudrich, and P. Pillet. Star cluster dynamics in a laboratory: electrons in an ultracold plasma. Mon. Not. R. Astron. Soc., 361(4):1227, 2005.

[48] G. Kalman, K. I. Golden, and M. Minella. Correlational and strong coupling effects on collective modes in plasmas. In H. M. Van Horn and S. Ichimaru, editors, Strongly Coupled Plasma Physics, page 323. University of Rochester Press, Rochester, 1993.

[49] S. D. Bergeson and R. L Spencer. Neutral-plasma oscillations at zero temperature. Phys. Rev. E, 67:026414, 2003.

[50] R. S. Fletcher, X. L. Zhang, and S. L. Rolston. Observation of collective modes of ultracold plasmas. Phys. Rev. Lett., 96:105003, 2006.

[51] S. B. Nagel, C. E. Simien, S. Laha, P. Gupta, V. S. Ashoka, and T. C. Killian. Magnetic trapping of metastable ${ }^{3} P_{2}$ atomic strontium. Phys. Rev. A, 67:011401, 2003.

[52] H. J. Metcalf and P. van der Straten. Laser Cooling and Trapping. SpringerVerlag, New York, New York, 1999.

[53] M. Walhout, H. J. L. Megens, A. Witte, and S. L. Rolston. Magneto-optical trapping of metastable xenon: Isotope-shift measurements. Phys. Rev. A, 48(2):879, 1993. 
[54] T. C. Killian, Y. C. Chen, P. Gupta, S. Laha, Y. N. Martinez, P. G. Mickelson, S. B. Nagel, A. D. Saenz, and C. E. Simien. Absorption imaging and spectroscopy of ultracold neutral plasmas. J. Phys. B: At. Mol. Opt. Phys., 38:351, 2005. (Equations 7, 10, 11, and 17 should be multiplied by $\gamma_{0} / \gamma_{\text {eff }}$.).

[55] A. Walz-Flannigan, J. R. Guest, J.-H. Choi, and G. Raithel. Cold-Rydberg-gas dynamics. Phys. Rev. A, 69:63405, 2004.

[56] M. P. Robinson, B. L. Tolra, M. W. Noel, T. F. Gallagher, and P. Pillet. Spontaneous evolution of Rydberg atoms into an ultracold plasma. Phys. Rev. Lett., 85(21):4466, 2000.

[57] D. Ciampini, M. Anderlini, J. H. Muller, F. Fuso, O. Morsch, J. W. Thomsen, and E. Arimondo. Photoionization of ultracold and Bose-Einstein-condensed Rb atoms. Phys. Rev. A, 66(4):043409, 2002.

[58] T. F. Gallagher. Rydberg Atoms. Cambridge University Press, 1994.

[59] W. Mende, K. Bartschat, and M. Koch. Near-threshold photoionization from the Sr I (5s5p) ${ }^{1} \mathrm{P}_{1}^{o}$ state. J. Phys. B: At. Mol. Opt. Phys., 28(12):2385, 1995.

[60] Jr. L. Spitzer. Physics of Fully Ionized Gases. Wiley, New York, 1962.

[61] S. L. Rolston, S. D. Bergeson, S. Kulin, and C. Orzel. A strongly coupled plasma or a dense Rydberg gas? Bull. Am. Phys. Soc., 43:1324, 1984.

[62] P. P. Edwards and C. N. R. Rao, editors. Metal-Insulator Transitions Revisited. Taylor \& Francis Ltd., 1995.

[63] G. Vitrant, J. M. Raimond, M. Gross, and S. Haroche. Rydberg to plasma evolution in a dense gas of very excited atoms. J. Phys. B, 15:49, 1982.

[64] W. Li, M. W. Noel, M. P. Robinson, P. J. Tanner, T. F. Gallagher, D. Comparat, B. Laburthe Tolra, N. Vanhaecke, T. Vogt, N. Zahzam, P. Pillet, and D. A. Tate. Evolution dynamics of a dense frozen Rydberg gas to plasma. Phys. Rev. A, 70:042713, 2004.

[65] D. Jaksch, J. I. Cirac, P. Zoller, S. L. Rolston, R. Côté, and M. D. Lukin. Fast quantum gates for neutral atoms. Phys. Rev. Lett., 85(10):2211, 2000.

[66] T. C. Killian, M. J. Lim, S. Kulin, R. Dumke, S. D. Bergeson, and S. L. Rolston. Formation of Rydberg atoms in an expanding ultracold neutral plasma. Phys. Rev. Lett., 86(17):3759, 2001.

[67] J. L. Roberts, C. D. Fertig, M. J. Lim, and S. L. Rolston. Electron temperature of ultracold plasmas. Phys. Rev. Lett., 92:253003, 2004.

[68] N. Vanhaecke, D. Comparat, D. A. Tate, and P. Pillet. Ionization of Rydberg atoms embedded in an ultracold plasma. Phys. Rev. A, 71:013416, 2005.

[69] M. S. Murillo. Critical wave vectors for transvers modes in strongly coupled dusty plasmas. Phys. Rev. Lett., 85(12):2514, 2000. 
[70] E. A. Cummings, J. E. Daily, D. S. Durfee, and S. D. Bergeson. Ultracold neutral plasma expansion in two dimensions. Phys. Plasmas, 12:123501, 2005.

[71] S. Laha, Y. C. Chen, P. Gupta, C. E. Simien, Y. N. Martinez, P. G. Mickelson, S. B. Nagel, and T. C. Killian. Kinetic energy oscillations in annular regions of ultracold neutral plasmas. Euro. Phys. J. D, 40:51, 2006.

[72] A. E. Siegman. Lasers. University Science Books, Sausolito, California, 1986.

[73] T. Pohl, T. Pattard, and J. M. Rost. Kinetic modeling and molecular dynamics simulation of ultracold neutral plasmas including ionic correlations. Phys. Rev. A, 70(3):033416, 2004.

[74] Z. Donkó, B. Nyíri, L. Szalai, and S. Holló. Thermal conductivity of the classical electron one-component plasma. Phys. Rev. Lett., 81(8):1622, 1998.

[75] J. P. Hansen. Statistical mechanics of dense ionized matter. I. Equilibrium properties of the classical one-component plasma. Phys. Rev. A, 8:3096, 1973.

[76] H. E. DeWitt. Asymptotic form of the classical one-component plasma fluid equation of state. Phys. Rev. A, 14(3):1290, Sep 1976.

[77] A. Y. Potekhin, G. Chabrier, and Y. A. Shibanov. Partially ionized hydrogen plasma in strong magnetic fields. Phys. Rev. E, 60:2193, 1999.

[78] J. Vorberger, M. Schlanges, and W. D. Kraeft. Equation of state for weakly coupled quantum plasmas. Phys. Rev. E, 69:046407, 2004.

[79] A. V. Filinov, V. O. Golubnychiy, M. Bonitz, W. Ebeling, and J. W. Dufty. Temperature-dependent quantum pair potentials and their application to dense partially ionized hydrogen plasmas. Physical Review E, 70:046411, 2004.

[80] G. Zwicknagel. Molecular dynamics simulations of the dynamics of correlations and relaxation in an OCP. Contrib. Plasma Phys., 39:155, 1999.

[81] K. Morawetz, M. Bonitz, V. G. Morozov, G. Röpke, and D. Kremp. Short-time dynamics with initial correlations. Phys. Rev. E, 63:020102, 2001.

[82] R. Redmer. Physical properties of dense, low-temperature plasmas. Phys. Rep., 282:36, 1997.

[83] A. Ng, A. Forsman, and G. Chiu. Electron thermal conduction waves in a two-temperature, dense plasma. Phys. Rev. Lett., 81(14):2914, 1998.

[84] Y.L. Klimontovich and D. Kremp. Quantum kinetic-equations in systems with bound states. Physica A, 109:517, 1981.

[85] M. Surendra, D. B. Graves, and G. M. Jellum. Self-consistent model of a directcurrent glow discharge: Treatment of fast electrons. Phys. Rev. A, 41:1112, 1990.

[86] C.K. Birdsall. Particle-in-cell charged-particle simulations, plus monte carlo collisions with neutral atoms, PIC-MCC. IEEE Trans. Plasma Sci., 19:65, 1991. 
[87] T. Ohde, M. Bonitz, T. Bornath, D. Kremp, and M. Schlanges. Twotemperature relaxation in nonideal partially ionized plasmas. Phys.Plasmas, 3:1241, 1996.

[88] M. R. Flannery and D. Vrinceanu. Quantal and classical radiative cascade in Rydberg plasmas. Phys. Rev. A, 68:030502, 2003.

[89] R.L. Liboff. Kinetic Theory: Classical, Quantum, and relativistic descriptions. Wiley, New York, 1998.

[90] A. V. Gurevich, L. V. Pariskaya, and L. P. Pitaievskii. Selfsimilar motion of rarefield plasmas. Sov. Phys. JETP, 22:449, 1966.

[91] C. Sack and H. Schamel. Plasma expansion into vacuum - A hydrodynamic approach. Phys. Rep., 156:311, 1987.

[92] D. S. Dorozhkina and V. E. Semenov. Exact solution of Vlasov equations for quasineutral expansion of plasma bunch into vacuum. Phys. Rev. Lett., 81(13):2691, 1998.

[93] P. Mora. Plasma expansion into a vacuum. Phys. Rev. Lett., 90:185002, 2003.

[94] T. Pohl, T. Pattard, and J.M. Rost. Influence of electron-ion collisions on Coulomb crystallization of ultracold neutral plasmas. J. Phys. B, 38:S343, 2005.

[95] F. Robicheaux and J.D. Hanson. Simulation of the expansion of an ultracold neutral plasma. Phys. Rev. Lett., 88:055002, 2002.

[96] M. Bonitz. Correlation time approximation in non-Markovian kinetics. Phys. Lett. A, 221:85, 1996.

[97] D. H. E. Dubin and T. M. O'Neil. Trapped nonneutral plasmas, liquids, and crystals (the thermal equilubrium states). Rev. Mod. Phys., 71(1):87, 1999.

[98] W. L. Slattery, G. D. Doolen, and H. E. DeWitt. Improved equation of state for the classical one-component plasma. Phys. Rev. A, 21(6):2087, 1980.

[99] K.C. Ng. Hypernetted chain solutions for classical one-component plasma up to $\Gamma=7000$. J. Chem. Phys., 61:2680, 1974.

[100] G. Chabrier and A. Y. Potekhin. Equation of state of fully ionized electron-ion plasmas. Phys. Rev. E, 58(4):4941, 1998.

[101] S. Hamaguchi, R. T. Farouki, and D. H. E. Dubin. Phase diagram of Yukawa systems near the one-component-plasma limit revisited. J. Chem. Phys., 105(17):7641, 1996.

[102] S. Hamaguchi, R. T. Farouki, and D. H. E. Dubin. Triple point of yukawa systems. Phys. Rev. E, 56(4):4671, 1997.

[103] D. O. Gericke and M. S. Murillo. Disorder-induced heating of ultracold plasmas. Contrib. Plasma Phys., 43:298, 2003. 
[104] D. O. Gericke, M. S. Murillo, D. Semkat, M. Bonitz, and D. Kremp. Relaxation of strongly coupled Coulomb systems after rapid changes of the interaction potential. J. Phys. A, 36:6087, 2003.

[105] S. J. Aarseth. Star cluster simulations: The state of the art. Celest. Mech. Dyn. Astr., 73:137, 1999.

[106] S. X. Hu, D. Vrinceanu, S. Mazevet, and L. A. Collins. Molecular-dynamics simulations of cold antihydrogen formation in strongly magnetized plasmas. Phys. Rev. Lett., 95(16):163402, 2005.

[107] T. Pohl, H.R. Sadeghpour, and G. Gabrielse. New interpretations of measured antihydrogen velocities and field ionization spectra. Phys. Rev. Lett., 97:143401, 2006.

[108] M. Tuckerman, B. J. Berne, and G.J. Martyna. Reversible multiple time scale molecular dynamics. J. Chem. Phys., 97(3):1990, 1992.

[109] R.W. Hockney and J.W. Eastwood. Computer Simulation Using Particles. Institute of Physics Publ., Bristol, 1994.

[110] S. J. Aarseth. Direct methods for n-body simulation. In J.U. Brackbill and B.I. Cohen, editors, Multiple Time Scales, New York, 1985. Academic Press.

[111] J. Barnes and P. Hut. A hierarchical $\mathrm{O}(N \log N)$ force-calculation algorithm. Nature, 324:446, 1986.

[112] L. Greengard and V. Rokhlin. A fast algorithm for particle simulations. $L$. Comp. Phys., 73:325, 1987.

[113] C.K. Birdsall and A.B. Langdon. Plasma Physics via Computer Simulations. Institute of Physics Publ., London, 1995.

[114] M.M. Shoucri O.V. Batishchev and A.A. Batishcheva. Fully kinetic simulation of coupled plasma and neutral particles in scrape-off layer plasma of fusion devices. J. Plasma Phys., 61:347, 1999.

[115] G. Zhao and L. Chen. Gyrokinetic particle-in-cel 1 simulation of alvenic ion temperature-gradient modes in tokamak plasma. Phys. Plasma, 9:2926, 2002.

[116] W.J. Goedheer and Y.I. Chutov. Pic/mc modeling of dusty radio-frequency discharges. IEEE Trans. Plasma Sc., 32:551, 2004.

[117] I.V. Schweigert and V.A. Schweigert. Combined pic-mcc approach for fast simulation of a radio frequency discharge at a low gas pressure. Plasma Sources Sc. and Tech., 13:315, 2004.

[118] M. Surendra. Radiofrequency discharge benchmark model comparison. Plasma Sources Sc. and Tech., 4:56, 2004.

[119] J.P. Verboncoeur. Symmetric spline weighting for charge and current density in particle simulations. J. Comp. Phys., 174:421, 2001. 
[120] S. Chandrasekhar. Dynamical friction. iii. A more exact theory of the rate of escape of stars from clusters. Astrophys. J., 98:54, 1943.

[121] L. Spitzer and Härm. Evaporation of stars from isolated clusters. Astrophys. J., 127:544, 1958 .

[122] I.R. King. The structure of star clusters III. Some simple dynamical models. Astron. Journal, 71:64, 1966.

[123] R.W. Michie. On the distribution of high energy stars in spherical stellar systems. Monthly Notices Roy. Astron. Soc., 127:125, 1963.

[124] R. J. Goldston and P. H. Rutherford. Introduction to Plasma Physics. Institute of Physics, Philadelphia, 1995.

[125] F. F. Chen. Introduction to Plasma Physics. Plenum, New York, 1974.

[126] W. Li, P. J. Tanner, and T. F. Gallagher. Dipole-dipole excitation and ionization in an ultracold gas of Rydberg atoms. Phys. Rev. Lett., 94:173001, 2005.

[127] T. Pohl, T. Pattard, and J. M. Rost. Plasma formation from ultracold Rydberg gases. Phys. Rev. A, 68:010703, 2003.

[128] T. B. Mitchell, J. J. Bollinger, D. H. E. Dubin, X. -P. Huang, W. M. Itano, and R. H. Baughman. Direct observations of structural phase transitions in planar crystallized ion plasmas. Science, 282:1290, 1998.

[129] J. P. Hansen, E. L. Pollock, and I. R. McDonald. Velocity autocorrelation funtion and dynamical structure factor of the classical one-component plasma. Phys. Rev. Lett, 32(6):277, 1974.

[130] H. Gould and G. F. Mazenko. Coupling of single-particle and collective motions in a one-component plasma. Phys. Rev. Lett., 35(21):1455, 1975.

[131] M. Bonitz, D. Semkat, and D. Kremp. Short-time dynamics of correlated many-particle systems: Molecular dynamics versus quantum kinetics. Phys. Rev. E, 56(1):1246, 1997.

[132] I. V. Morozov and G. E. Norman. Non-exponential dynamic relaxation in strongly nonequilibrium nonideal plasmas. J. Phys. A: Math. Gen., 36:6005, 2003.

[133] T. Pohl, T. Pattard, and J. M. Rost. On the possibility of 'correlation cooling' of ultracold neutral plasmas. J. Phys. B: At. Mol. Opt. Phys., 37:183, 2004.

[134] R. T. Farouki and S. Hamaguchi. Thermodynamics of strongly-coupled Yukawa systems near the one-component-plasma limit. II. Molecular dynamics simultations. J. Chem. Phys., 101(11):9885, 1994.

[135] As the number of electrons per Debye sphere $\left(\kappa^{-3}\right)$ approaches unity, the Yukawa interaction ceases to accurately describe ion-ion interactions. For strontium plasmas studied here, this situation only occurs for the highest $n_{e}$ and lowest $T_{e}$. 
[136] N. N. Bogoliubov. Problems of Dynamical Theory in Statistical Physics. Gostekhizdat, Moscow-Leningrad, 1946. reprinted in Studies in Statistical Mechanics, edited by J. de Boer and G.E. Uhlenbeck (Amsterdam, NorthHolland, 1962), Vol. 1.

[137] J. P. Hansen, I. R. McDonald, and E. L. Pollock. Statistical mechanics of dense ionized matter. III. Dynamical properties of the classical one-component plasma. Phys. Rev. A, 11(3):1025, 1975.

[138] L. Tonks and I. Langmuir. Oscillations in ionized gases. Phys. Rev., 33(2):195, 1929.

[139] J. V. Parker, J. C. Nickel, and R. W. Gould. Resonance oscillations in a hot nonuniform plasma. Phys. Fluids, 7(9):1489, 1964.

[140] D. Bohm and E. P. Gross. Theory of plasma oscillations. a. Origin of mediumlike behavior. Phys. Rev., 75(12):1851, 1949.

[141] N. Herlofson. Plasma resonance in ionospheric irregularities. Arkiv Fysik, 3:247, 1951.

[142] A. Dattner. Resonance densities in a cylindrical plasma column. Phys. Rev. Lett., 10(6):205, 1963.

[143] V. F. Kovalev and V. Yu. Bychenkov. Analytic solutions to the Vlasov equations for expanding plasmas. Phys. Rev. Lett., 90(18):185004, 2003.

[144] F. Cornolti, F. Ceccherini, S. Betti, and F. Pegoraro. Charged state of a spherical plasma in vacuum. Phys. Rev. E, 71(5):056407, 2005.

[145] P. Mora. Plasma expansion into a vacuum. Phys. Rev. Lett., 90:185002, 2003.

[146] E. L. Clark, K. Krushelnick, J. R. Davies, M. Zepf, M. Tatarakis, F. N. Beg, A. Machacek, P. A. Norreys, M. I. K. Santala, I. Watts, and A. E. Dangor. Measurements of energetic proton transport through magnetized plasma from intense laser interactions with solids. Phys. Rev. Lett., 84(4):670, 2000.

[147] M. Tribeche, H. Houili, and T. H. Zerguini. Expansion of dust grains in electron depleted two-temperature-ion plasmas. Phys. Plasmas, 11(6):3001, 2004.

[148] Y. Hahn. Relaxation of cold plasmas and threshold lowering effect. Phys. Rev. E, 64:046409, 2001.

[149] A.V. Gurevich, L.V. Pariiskaya, and L.P. Pitaevskii. Selfsimilar motion of rarefied plasma. Sov. Phys. JETP, 22:449, 1966.

[150] C. Sack and H. Schamel. Evolution of a plasma expanding into vacuum. Plasma. Phys. Contr. F., 27:717, 1985.

[151] A. N. Tkachev and S. I. Yakovlenko. Relaxation of rydberg states in an ultracold plasma. Quantum Electronics, 31(12):1084, 2001.

[152] M. J. Seaton. Radiative recombination of hydrogenic ions. Mon. Not. R. Astron. Soc., 119:81, 1959. 
[153] W. Graham, W. Fritsch, Y. Hahn, and J. A. Tanis, editors. NATO ASI Series B: Physics, Recombination of Atomic Ions, volume 296. Plenum, New York, 1992.

[154] Y. Hahn. Plasma density effects on the three-body recombination rate coefficients. Phys. Lett. A, 231:82, 1997.

[155] Y. Hahn. Improved rates for three-body recombination at low temperature. Phys. Lett. A, 264:465, 2000.

[156] S. A. Maiorov, A. N. Tkachev, and S. I. Yakovlenko. Metastable state of supercooled plasma. Physica Scripta, 51:498, 1998.

[157] H. Gao amd D. R. DeWitt, R. Schuch, W. Zong, S. Asp, and M. Pajek. Observation of enhanced electron-ion recombination rates at very low energies. Phys. Rev. Lett., 75:4381, 1995.

[158] M. Pajek and R. Schuch. Plasma effects in three-body recombination of high-z bare ions with electrons. Phys. Scr., T80:307, 1999.

[159] Y. Hahn. Threshold lowering effects on an expanding cold plasma. Phys. Lett. A, 293:266, 2002.

[160] S.L. Gilbert, J.J. Bollinger, and D.J. Wineland. Shell-structure phase of magnetically confined strongly coupled plasmas. Phys. Rev. Lett., 60:2022, 1988.

[161] W. M. Itano, J. J. Bollinger, J. N. Tan, B. Jellenkovic, X.-P. Huang, and D. J. Wineland. Bragg diffraction from crystallized ion plasmas. Science, 279:686, 1998.

[162] M. Drewsen, C. Brodersen, L. Hornekær, J. S. Hangst, and J. P. Schifffer. Large ion crystals in a linear Paul trap. Phys. Rev. Lett., 81:2878, 1998.

[163] Niels K. and M. Drewsen. Observation of a structural transition for Coulomb crystals in a linear Paul trap. Phys. Rev. Lett., 91(9):095002, 2003.

[164] M.J. Jensen, T. Hasegawa, J.J. Bollinger, and D.H.E. Dubin. Rapid heating of a strongly coupled plasma near the solid-liquid phase transition. Phys. Rev. Lett., 94:025001, 2005.

[165] D.H.E. Dubin. Measurement of screening enhancement to nuclear reaction rates using a strongly magnetized and strongly correlated non-neutral plasma. Phys. Rev. Lett., 94:025002, 2005.

[166] H. Thomas, G. E. Morfill, V. Demmel, J. Goree, B. Feuerbacher, and D. Möhlmann. Plasma crystal: Coulomb crystallization in a dusty plasma. Phys. Rev. Lett., 73:652, 1994.

[167] O. Arp, D. Block, Al. Piel, and A. Melzer. Dust Coulomb balls: Threedimensional plasma crystals. Phys. Rev. Lett., 93(16):165004, 2004. 
[168] M. Bonitz, D. Block, O. Arp, V. Golubnychiy, H. Baumgartner, P. Ludwig, A. Piel, and A. Filinov. Structural properties of screened Coulomb balls. Phys. Rev. Lett., 96(7):075001, 2006.

[169] M. Bonitz, V. S. Filinov, V. E. Fortov, P. R. Levashov, and H. Fehske. Crystallization in two-component Coulomb systems. Phys. Rev. Lett., 95(23):235006, 2005.

[170] T. C. Killian, V. S. Ashoka, P. Gupta, S. Laha, S. B. Nagel, C. E. Simien, S. Kulin, S. L. Rolston, and S. D. Bergeson. Ultracold neutral plasmas: recent experiments and new prosects. J. Phys. A: Math. Gen., 36:6077, 2003.

[171] G. Birkl, S. Kassner, and H. Walther. Multiple-shell structure of laser-cooled ${ }^{14} \mathrm{Mg}^{+}$ions in a quadrupole storage ring. Nature, 357:310, 1992.

[172] J.J. Bollinger, J.M. Kriesel, T.B. Mitchell, L.B. King, M.J. Jensen, W.M. Itano, and D.H.E. Dubin. Laser-cooled ion plasmas in Penning traps. J. Phys. $B, 36: 499,2003$.

[173] L. Hornekær and M. Drewsen. Formation process of large ion Coulomb crystals in linear Paul traps. Phys. Rev. A, 66:013412, 2002.

[174] D.H.E. Dubin and T.M. O'Neil. Computer simulation of ion clouds in a Penning trap. Phys. Rev. Lett., 60:511, 1988.

[175] J.P. Schiffer. Order in confined ions. J. Phys. B, 36:511, 2003.

[176] H. Totsuji and J.L. Barrat. Structure of a nonneutral classical plasma in a magnetic field. Phys. Rev. Lett., 60:2484, 1988.

[177] T. Pohl, D. Comparat, N. Zahzam, T. Vogt, P. Pillet, and T. Pattard. Use of Rydberg atoms to control electron temperatures in ultracold plasmas. Eur. Phys. J. D, 39:45, 2006.

[178] S. Hamaguchi, R. T. Farouki, and D. H. E. Dubin. Thermodynamics of stronlgy-coupled Yukawa systems near the one-component-plasma limit. i. Derivation of excess energy. J. Chem. Phys., 101(11):9876, 1994.

[179] J.-H. Choi, J.R. Guest, A.P. Povilus, E. Hansis, and G. Raithel. Magnetic trapping of long-lived cold Rydberg atoms. Phys. Rev. Lett., 95:243001, 2005.

[180] J.-H. Choi, J.R. Guest, E. Hansis, A.P. Povilus, and G. Raithel. Landau quantization and time dependence in the ionization of cold, strongly magnetized Rydberg atoms. Phys. Rev. Lett., 95:253005, 2005.

[181] F. Robicheaux. Simulations of antihydrogen formation. Phys. Rev. A, 70:022510, 2004.

[182] J. R. Guest, J.-H. Choi, and G. Raithel. Decay rates of high- $|m|$ Rydberg states in strong magnetic fields. Phys. Rev. A, 68:022509, 2003.

[183] T. Topcu and F. Robicheaux. Radiative cascade of highly excited hydrogen atoms in strong magnetic fields. Phys. Rev. A, 73:043405, 2006. 
[184] T. Pohl, H. R. Sadeghpour, Y. Nagata, and Y. Yamazaki. Cooling by spontaneous decay of highly excited antihydrogen atoms in magnetic traps. Phys. Rev. Lett., 97:213001, 2006. 